\title{
Corticosterone secretion in tuatara (Sphenodon punctatus): Influential factors and conservation applications
}

\author{
Lindsay Erin Anderson
}

A thesis submitted to Victoria University of Wellington in fulfilment of the requirements for the degree of Doctor of Philosophy in Ecology and Biodiversity

Victoria University of Wellington

Te Whare Wānanga o te Ūpoko o te Ika a Mãui 



\section{ABSTRACT}

Animals are regularly exposed to environmental, social and physiological challenges. In reaction to these challenges, individuals adjust their physiology and behaviour to maintain essential processes and optimise fitness. The most widely used indicators of physiological stress in vertebrates are glucocorticoid hormones (corticosterone (CORT) or cortisol), which are commonly referred to as 'stress hormones'. The use of CORT as a tool to understand how individuals respond to natural or human-caused challenges is central to stress physiology research. Here, I investigated intrinsic and extrinsic factors associated with CORT secretion, CORT secretion as an indicator of physiological response to challenges/stressors, and the value of CORT secretion as conservation tool in an iconic protected reptile (the tuatara, Sphenodon punctatus).

A capture-restraint time series revealed a significant CORT response over a $24 \mathrm{~h}$ period in male and female (non-gravid and gravid) tuatara. Baseline CORT and the CORT response to capture and restraint (i.e. a standardised capture-stress protocol) were similar between sexes; however, female reproductive condition was correlated with CORT secretion in that higher baseline CORT and a lower CORT response were observed in gravid females. An observational study incorporating data across a wide range of ambient temperatures (from four island sites) confirmed that body temperature $\left(T_{\mathrm{b}}\right)$ is positively correlated with baseline CORT in gravid females only, and revealed a positive correlation between the CORT response and higher $T_{\mathrm{b}}$ in all adults. A supporting experimental study showed that acute ambient temperature increase (in which mean $T_{\mathrm{b}}$ reached $21.4 \pm 0.4^{\circ} \mathrm{C}$ ) elicits a significant CORT response to capture-restraint in gravid females. These results confirmed that gravid females are not secreting CORT maximally during nesting, but actively modulate secretion. An inter-island comparison of CORT secretion (for four populations) revealed that baseline CORT secretion was similar among populations during the non-breeding and breeding seasons; however, the CORT response to capture-restraint varied significantly among populations. Inter-population variation in testosterone ( $\mathrm{T}$ ) was 
observed in males (but not females) and was positively linked with increased baseline CORT from the non-breeding season to the breeding season, suggesting male reproductive activity may drive seasonal change of baseline CORT. Significant correlations were observed between the CORT response to capture-restraint (but not baseline CORT) and habitat elements of latitude, tuatara density and seabird abundance and 2) demogenetic factors of sex ratio and genetic diversity. The measurement of CORT as a physiological monitoring tool indicated that short- and long- term dynamics of CORT secretion in tuatara are not altered through translocation to a new island, as the acute CORT response remained stable throughout exposure to cumulative stressors and long-term dynamics of CORT secretion in translocated populations simultaneously mirrored those in source populations.

These findings deliver the most detailed study of CORT secretion patterns in tuatara to date. Moreover, as the first study to apply CORT secretion data as an conservation physiology monitoring tool in tuatara, these results serve as a baseline reference for future research and monitoring of conservation efforts. 


\section{ACKNOWLEDGEMENTS}

I have had such a great time during my PhD studies and have learned so much. First of all, I am extremely grateful to my supervisors Nicky Nelson, Alison Cree and Dave Towns for their wisdom, encouragement, guidance and feedback throughout it all!

My research was made possible through support from the Zoological Society of San Diego, the Society for Research on Amphibians and Reptiles in New Zealand, the Allan Wilson Centre for Molecular Ecology and Evolution, the Centre for Biodiversity and Restoration Ecology and Victoria University of Wellington. This work was supported by Ngātiwai iwi , Ngāti Kōata iwi, Te Ātiawa Manawhenua Ki Te Tau Ihu iwi and was carried out in accordance with permits from the New Zealand Department of Conservation (35087-FAU, AK-33028-FAU, NO-30504-FAU) and from the animal ethics committee of Victoria University of Wellington (AEC protocol \# 2009R12).

A big thank you goes out to Danielle Middleton, who trained me how to collect a blood sample from a tuatara tail vein (not an easy task!). I'm so grateful to Sue Keall, who showed me the ropes on my first field trip and accompanied me on many others - you made the torturous nightly climbs up Taranga Island bearable! So many great people volunteered their time to help me with my fieldwork. Thanks to Sean Anderson, Tire Apetera Wills, Steph Borrelle, Kieran Campbell, Anna Carter, Katherine Cole, Whiria Fletcher, Richard Gibson, Kristine Grayson, Korora Hotorene, Kalin Lewis, Noela McGregor, Danielle Middleton, Pete Mitchell, Cathy Mitchell, Steph Price, Mark Pugsley, Edward Ramirez, Helen Taylor, Colin Thompson, Graham Ussher, Matthew Williams, and volunteers from the Auckland Zoo and Auckland University of Technology.

Thanks to Sophie Penniket, Anne Besson, Stephen Adolph, Sophie Gibson, and Scott Jarvie for making me feel so welcome at the University of Otago, sharing your office 
space, and including me in your lab group. Thanks also go to laboratory/support staff at the University of Otago for providing laboratory and administrative assistance. Many thanks are owed to Professor Helen Nicholson at the University of Otago (School of Medical Sciences) for providing tritiated testosterone. The School of Biological Sciences administration team at Victoria University of Wellington has also been a huge support; thank you Mary Murray, Sandra Taylor, Paul Marsden and Patricia Stein.

Thank you to all my friends, both in New Zealand and back home in Canada, for encouraging me in my studies, listening to me rant and babble on about writing, and joining me for a much-needed coffee or pint. Cheers to Kevin Bohnet, Danielle Campbell, Shawn Campbell, Kieran Campbell, Jo Carpenter, Anna Carter, Jeanine Clements, Curtis Clements, Ilse Corkery, Eda Czarnecki, Clay Gordon, Kristine Grayson, Mike Knapik, Kat Lee, Amelia MacRae, Danielle Middleton, Grace Paske, Keely Portsmouth, Helen Taylor and Leighton Thomas. Special thanks to James Mcvay and Garth Mowat for all your help at the end!

I am so grateful for the love and support from my family, my mom Linda (and Co), my sisters Kristin and Dayna (Aron), my nephews Cameron, Owen and Liam, and from Abby (I miss you!). Mom, Dayna \& Aron, Auntie Lee, Mark, Kat and Kieran your visits to New Zealand brought home a little closer.

Lastly, I don't think any words can describe the gratitude I feel for my husband Sean, who has left his job (twice!) to join me on my academic pursuits all around the world. Thank you for your constant support, understanding, patience, kindness and hugs. I am so lucky. 
To my mom, Linda 


\section{TABLE OF CONTENTS}

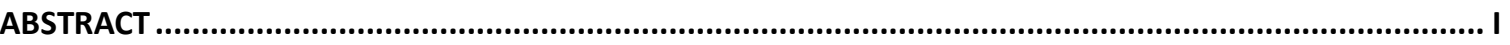

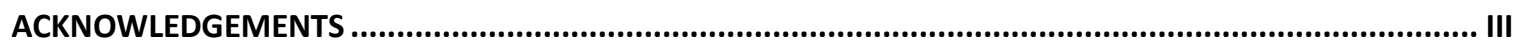

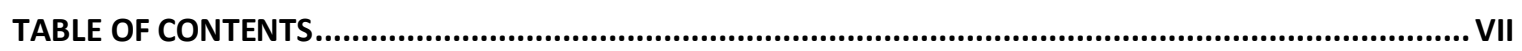

1 BIOLOGY OF GLUCOCORTICOIDS AND THEIR APPLICATION IN CONSERVATION: AN OVERVIEW .......... 1

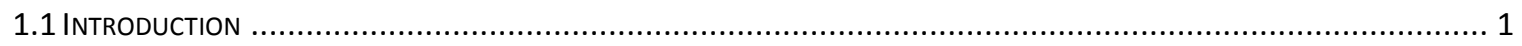

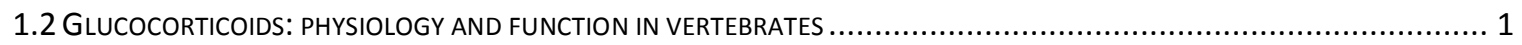

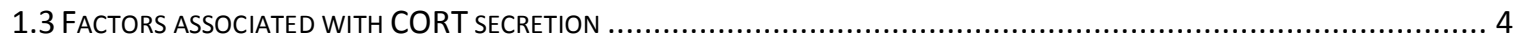

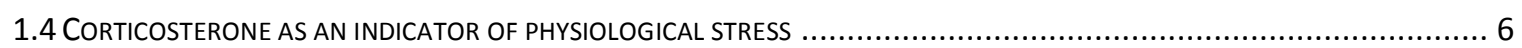

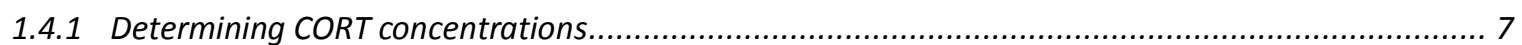

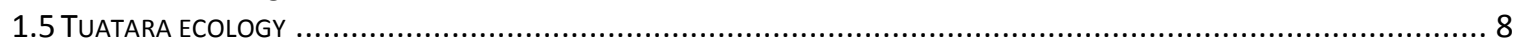

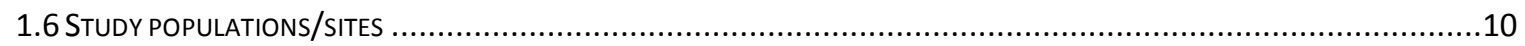

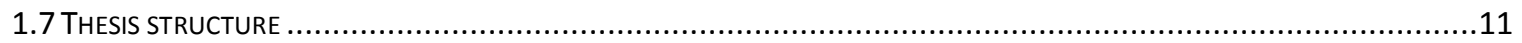

2 MODULATION OF CORTICOSTERONE SECRETION IN TUATARA (SPHENODON PUNCTATUS): EVIDENCE OF A DAMPENED STRESS RESPONSE IN GRAVID FEMALES ...........................................................15

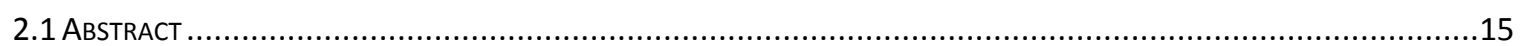

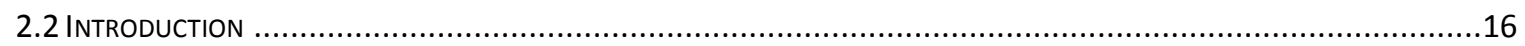

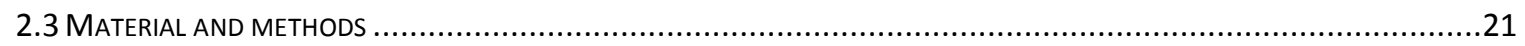

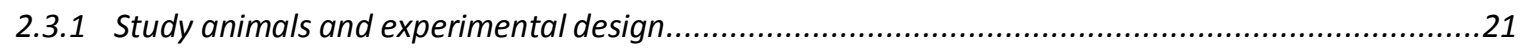

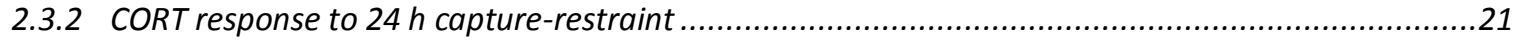

2.3.3 Internal body temperature and body-condition measurements.................................................22

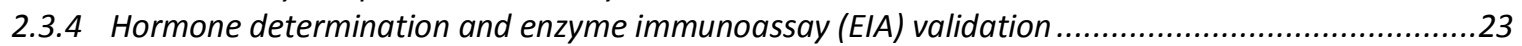

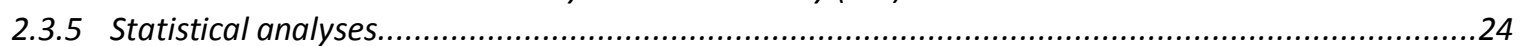

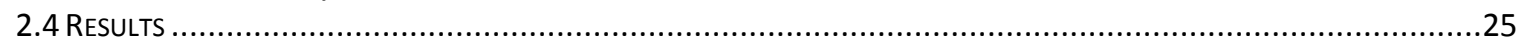

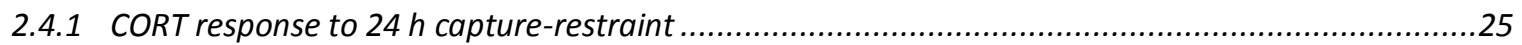

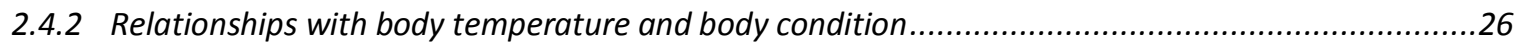

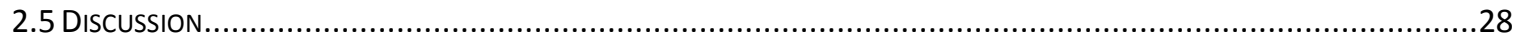

2.6 CONCLUSIONS

3 BODY TEMPERATURE IS CORRELATED WITH THE CORTICOSTERONE STRESS RESPONSE IN TUATARA

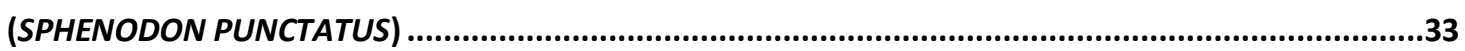

3.1 ABSTRACT

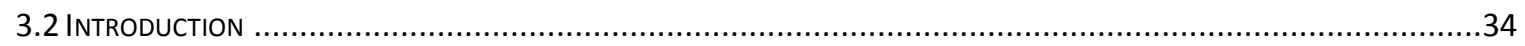

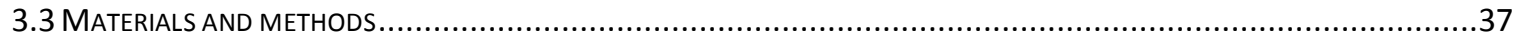

3.3.1 Influence of natural variation in body temperature on CORT secretion ...........................................37

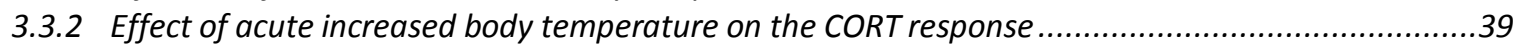

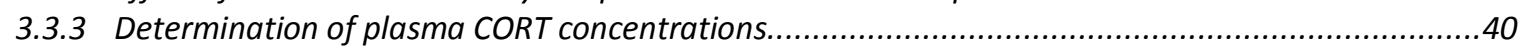

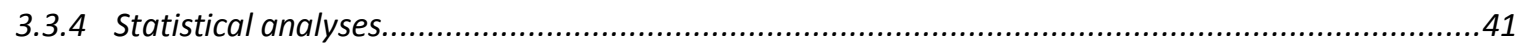

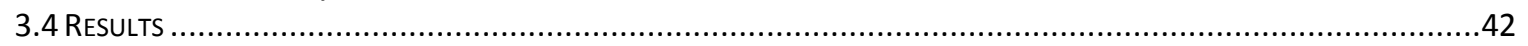

3.4.1 Influence of natural variation in body temperature on CORT secretion ..........................................42

3.4.2 Effect of acute increased body temperature on the CORT response ............................................45

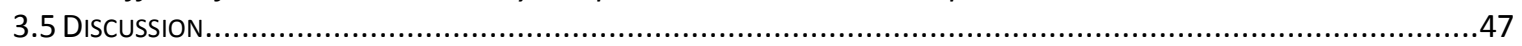

3.5.1 Body temperature is correlated with baseline CORT in gravid females only ..................................48

3.5.2 The CORT response is higher at warmer ambient temperatures .................................................48

3.5.3 Acute increased body temperature elicits a CORT response in gravid females ................................49

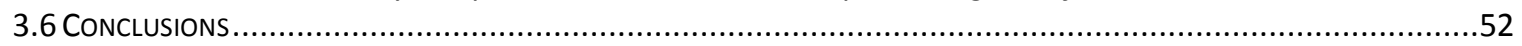


4 THE CORTICOSTERONE STRESS RESPONSE VARIES AMONG ISLAND POPULATIONS OF TUATARA (SPHENODON PUNCTATUS) AND IS ASSOCIATED WITH LINEAR ECOLOGICAL ATTRIBUTES................53

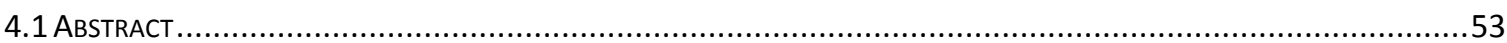

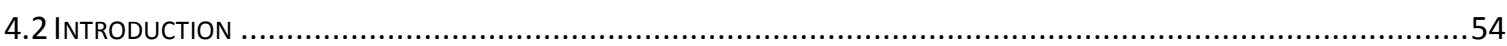

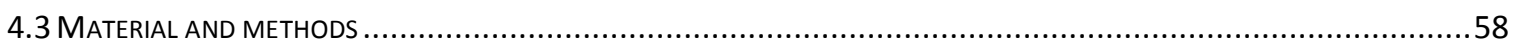

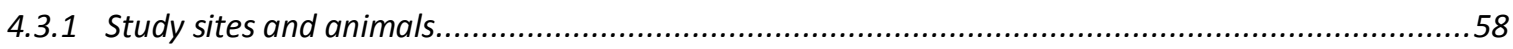

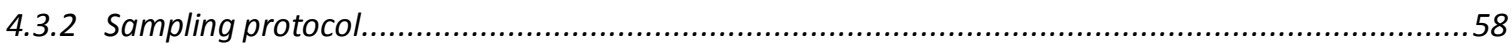

4.3.3 Linear ecological predictors: Principal components analysis (PCA) of ecological attributes ............59

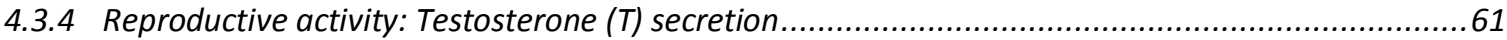

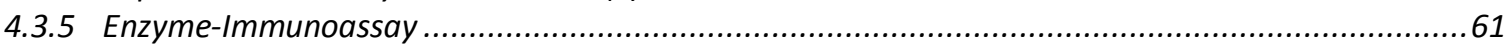

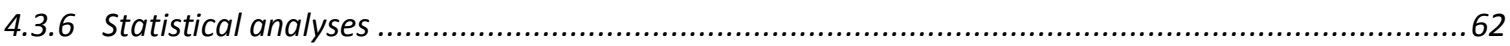

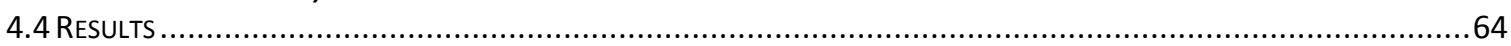

4.4.1 CORT secretion and body condition ...............................................................................64

4.4.2 Linear ecological predictors of CORT secretion (PCA axes) ................................................. 118

4.4.3 Reproductive activity: Testosterone $(T)$ secretion during the breeding season ...........................126

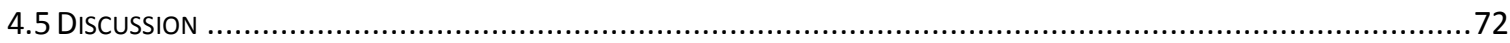

4.5.1 The CORT response and body condition vary among populations, but baseline CORT does not ....... 74

4.5.2 Relationships between The CORT response and linear ecological attributes (PC1 and PC2 scores) 132

4.5.3 Associations between reproductive activity $(T)$ and modulation of CORT secretion .......................137

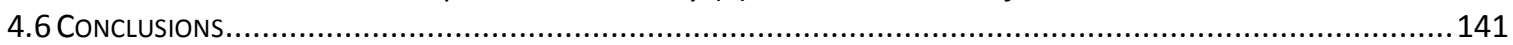

5 MOVING HOUSE: LONG-TERM DYNAMICS OF CORTICOSTERONE SECRETION ARE UNALTERED IN TRANSLOCATED POPULATIONS OF A RARE REPTILE (THE TUATARA, SPHENODON PUNCTATUS).....143

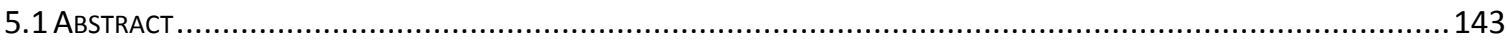

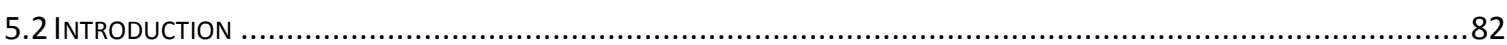

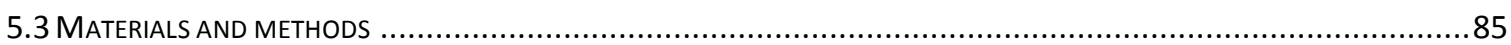

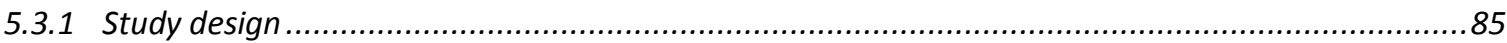

5.3.2 Short-term monitoring: The acute corticosterone response at different stages of the initial translocation process............................................................................................ 155

5.3.3 Long-term monitoring: Dynamics of corticosterone secretion post-translocation ........................88

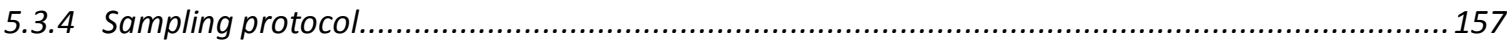

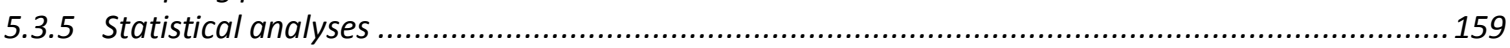

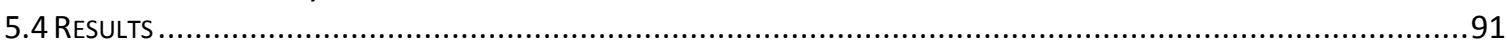

5.4.1 Short-term monitoring: The acute corticosterone response during different stages of the

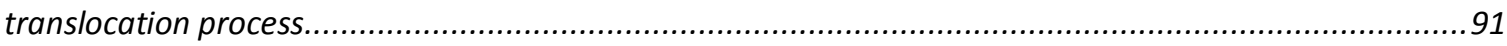

5.4.2 Long-term monitoring: Dynamics of corticosterone secretion in translocated populations ........... 164

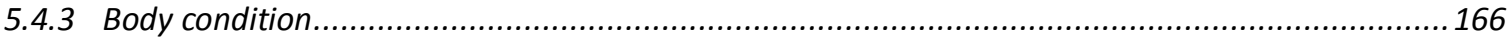

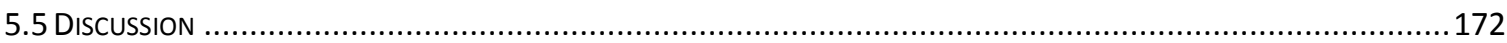

5.5.1 Cumulative stressors during translocation do not affect the acute CORT response in tuatara ....... 172

5.5.2 Long-term dynamics of CORT secretion in tuatara are not altered by translocation .......................174

\section{CORTICOSTERONE SECRETION IN TUATARA: THESIS SUMMARY AND CONSERVATION APPLICATIONS181}

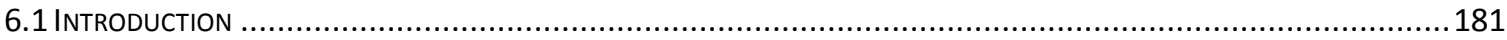

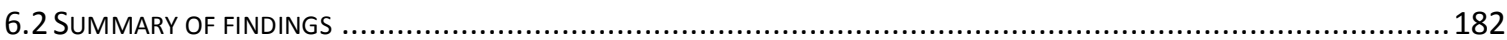

6.3 CONSERVATION IMPLICATIONS: THE VALUE OF CORT AS A CONSERVATION PHYSIOLOGY TOOL IN TUATARA...................106

6.3.1 Patterns of CORT secretion indicate a physiological response to challenges in tuatara ..................187

6.3.2 CORT secretion is a valuable addition to the conservation 'tool-box' for tuatara .........................190

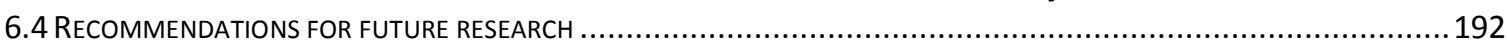

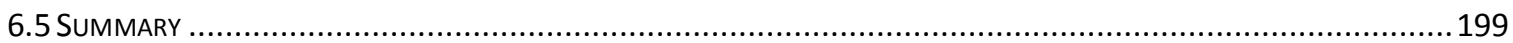

APPENDIX A: HORMONE MANIPULATION STUDIES IN GRAVID TUATARA........................................203

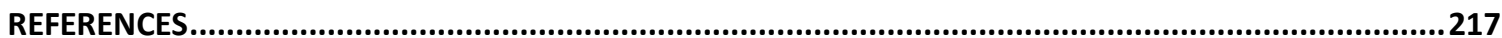




\section{Biology of glucocorticoids and their application in conservation: an overview}

\subsection{Introduction}

Human impacts continue to influence ecological change of natural ecosystems; therefore, it is becoming increasingly important to understand how organisms respond and cope with environmental change and unpredicted challenges (Wingfield, 2013). Physiological techniques have been utilised in ecological/conservation studies as tools to assess an individual's physiological response to challenges or a changing environment (Wikelski and Cooke, 2006). Understanding an individual's physiological response to certain challenges or stressors, and/or identifying the root of physiological stress, will enable development and consideration of more effective conservation management strategies and solutions (Dantzer et al., 2014; Madliger and Love, 2014). This thesis aims to advance the current understanding of reptile physiology by identifying intrinsic and extrinsic factors associated with the endocrine stress response in a rare reptile, the tuatara (Sphenodon punctatus), and subsequently examine its utility in monitoring conservation efforts. In this overview, I provide a brief background of glucocorticoid physiology, conservation physiology, and tuatara ecology to provide a general introductory framework for the four data chapters that follow.

\subsection{Glucocorticoids: physiology and function in vertebrates}

The endocrine system in vertebrates produces hormones that regulate and facilitate life-history transitions and responses to intrinsic and extrinsic changes/challenges 
(i.e. stressors), thereby playing an important fitness role (Crespi et al., 2013; Dufty et al., 2002; Jessop et al., 2013; Schultner et al., 2013). The hypothalamo-pituitaryadrenal (HPA) axis produces steroid hormones referred to as glucocorticoids (GCs) (Fig 1.1). At a basal level, GC secretion promotes essential life processes such as carbohydrate/ intermediary metabolism balance and cell provisioning of glucose (i.e. basic energy regulation). However, when an individual is exposed to unpredictable challenges/stressors, a complex adaptive hormonal response commonly referred to as the 'stress response' cascades through the HPA axis and stimulates further secretion of GCs (Busch and Hayward, 2009; Landys et al., 2006; Sapolsky et al., 2000)(Fig 1.1).

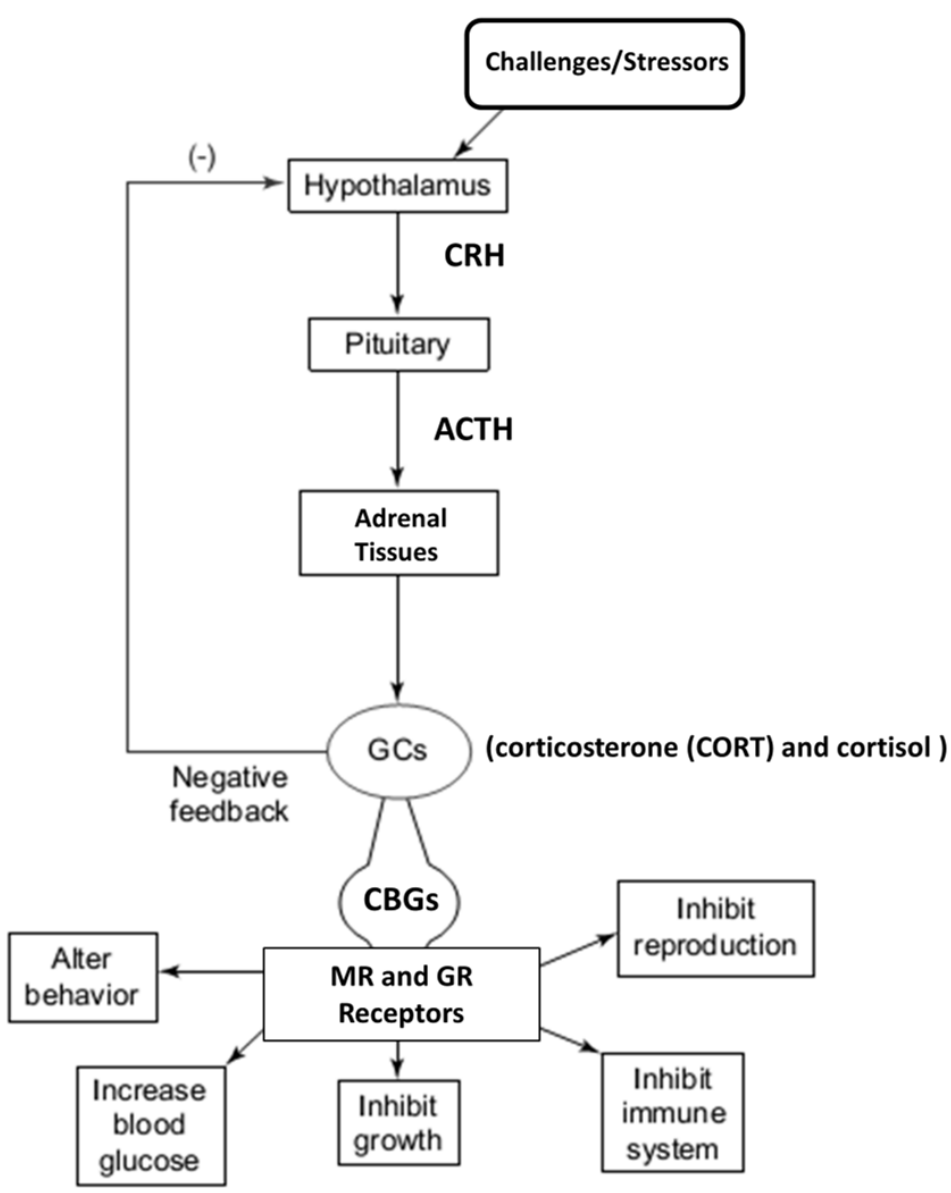

Figure 1.1. The vertebrate hypothalamo-pituitary-adrenal (HPA) axis. Increased glucocorticoid (GC) secretion (corticosterone (CORT) and cortisol) is activated through stimulation of the HPA axis in response to challenges/stressors. CORT is dispersed through the circulatory system by way of corticosteroid-binding globulins (CBGs). Figure modified from Romero (2004). 
The hypothalamus is stimulated in response to a challenge or stressor, triggering release of corticotropin-releasing hormone (CRH). In turn, $\mathrm{CRH}$ stimulates the pituitary to synthesise and release adrenocorticotropin hormone (ACTH), which then stimulates adrenal tissues to increase GC secretion well above basal levels (Sheriff et al., 2011) (Fig 1.1).

The primary GCs in vertebrates are corticosterone (CORT) and cortisol. CORT is dispersed through the circulatory system by way of corticosteroid-binding globulins (CBGs), and is delivered to specific target tissues, whereupon CORT binds to receptors (Schoech et al., 2013)(Fig 1.1). Receptors are widely distributed throughout cells in the body, and can therefore initiate a range of responses affecting behaviour, energy provision by stimulating gluconeogenesis (the formation of "new" glucose from non-carbohydrate sources), growth, immune response, and reproduction (Jessop et al., 2013; Romero, 2004) (Fig 1.1). CORT is modulated by two types of receptors: mineralocorticoid receptors (MR) and glucocorticoid receptors (GR). At lower CORT levels (i.e. basal CORT secretion), CORT preferentially binds to MRs (to regulate energy balance) and in contrast, GR receptors are bound when CORT concentrations are high (i.e. after a challenge/stressor) (Busch and Hayward, 2009; Romero, 2004). A negative feedback cycle regulates the continuation (or conversely discontinuation) of the CORT secretion process, and is based on the prevalence/status of the challenge or stressor (Romero, 2004; Sapolsky et al., 2000) (Fig 1.1).

Although GCs primarily function to support immediate survival and fitness, elevated GCs over extended periods of time (i.e. chronic stress) have been shown to have deleterious effects on an individual's overall health, condition and reproductive success (Tokarz and Summers, 2011). Harmful effects of elevated or sustained CORT secretion (both endogenous and exogenous) have been confirmed in a wide range of taxa. Examples include: decreased body condition in brown tree snakes (Boiga irregularis) (Waye and Mason, 2008), reduced bacterial killing ability and slower wound healing in marine iguanas (Amblyrhynchus cristatus) (French et al., 2010), reduced display and attack behaviour (DeNardo and Licht, 1993) and home 
range territories (DeNardo and Sinervo, 1994) in side-blotched lizards (Uta stansburiana).

Several studies have also shown that maternal CORT is transferred to young (in both viviparous and oviparous species) (Cree et al., 2003; Love et al., 2013; Uller et al., 2009). Hormones contribute to developmental plasticity in individuals and reproducing females exposed to stressors can affect morphological, physiological and behavioural traits in offspring that can subsequently affect their performance (Meylan et al., 2010; Michel et al., 2011). For example, stressed barn swallows (Hirundo rustica) (exposed to predators) laid eggs that had higher CORT than unstressed controls (exposed to herbivores) and eggs with higher CORT had lower hatching success and produced smaller hatchlings with slower plumage development, compared to controls (Saino et al., 2005). Pregnant female garter snakes (Thamnophis elegans) with elevated CORT produced offspring that exhibited decreased anti-predator behaviour (Robert et al., 2009). Activity (Belliure et al., 2004) and sprint speed/motivation to run (Meylan and Clobert, 2004) were decreased in common lizard (Lacerta vivipara) hatchlings produced by mothers with elevated CORT. Recent studies also demonstrate that CORT can influence sex determination of embryos. For example, in two lizard species that have temperature-dependent sex determination (TSD), elevated CORT altered sex-ratios in offspring; more males were produced in Bassiana duperreyi and more females were produced in Amphibolurus muricatus at pivotal temperatures (normally producing a 1:1 sex ratio). Sex-specific embryonic mortality was affected by CORT levels in A. muricatus, but not in B. duperreyi (Warner et al., 2009).

\subsection{Factors associated with CORT secretion}

It is generally agreed that baseline CORT serves to meet the energetic demands associated with everyday life, whereas the acute CORT response is a reaction to unpredictable and/or challenging events (Fig 1.2) (Tokarz and Summers, 2011; Wingfield et al., 1998). The acute CORT response allows individuals to react to a stressor by way of an adaptive physiological and/or behavioural response, and serves to promote overall fitness (Breuner et al., 2008; Busch and Hayward, 2009). 
Modulation of CORT secretion (i.e. daily and/or seasonal)(Fig 1.2) occurs and helps individuals cope with their specific environment to effectively increase fitness (Moore and Jessop, 2003; Romero, 2002).

Baseline CORT is commonly elevated during the breeding season to support energetic requirements associated with reproductive activities (such as nesting and mating), and similarly, individuals experiencing reduced breeding opportunities or living in harsh environments have been found to dampen their CORT response during the breeding season, which may serve to increase chances of reproductive success (Ebensperger et al., 2013; Holding et al., 2014b; Moore and Jessop, 2003; Romero, 2006; Wingfield et al., 1998; Wingfield and Sapolsky, 2003). For example, sea turtles display a reduced CORT response during nesting, even when faced with challenges such as non-lethal shark attacks and overheating, suggesting a trade-off of immediate survival for fitness (Jessop et al., 2004a; Jessop et al., 2000).

Patterns of CORT secretion are not universally consistent; variation is observed among species and within/among populations (Baker et al., 2013; Cockrem, 2013; Creel et al., 2013; Love et al., 2013). Variation in CORT secretion can be linked with intrinsic factors such as sex, reproductive status, body condition, and age (reviewed in Baker et al. (2013) and Cockrem (2013)). Also, extrinsic factors such as habitat modification - both natural and human-induced (Berner et al., 2013; French et al., 2010; Owen et al., 2014; Tartu et al., 2014; Taylor et al., 2014), seasonal/annual changes in food availability (Bryan et al., 2014; Kitaysky et al., 2007; Woodley et al., 2003), and fluctuating environmental temperature and/or weather (Lance et al., 2010; Telemeco and Addis, 2014) can influence CORT secretion. Furthermore, increased energy demands required during reproduction (Woodley and Moore, 2002), territorial disputes (Baird et al., 2014; Yang and Wilczynski, 2003) and migration (Hamann et al., 2007; Jessop et al., 2004b) can influence patterns of CORT secretion. 


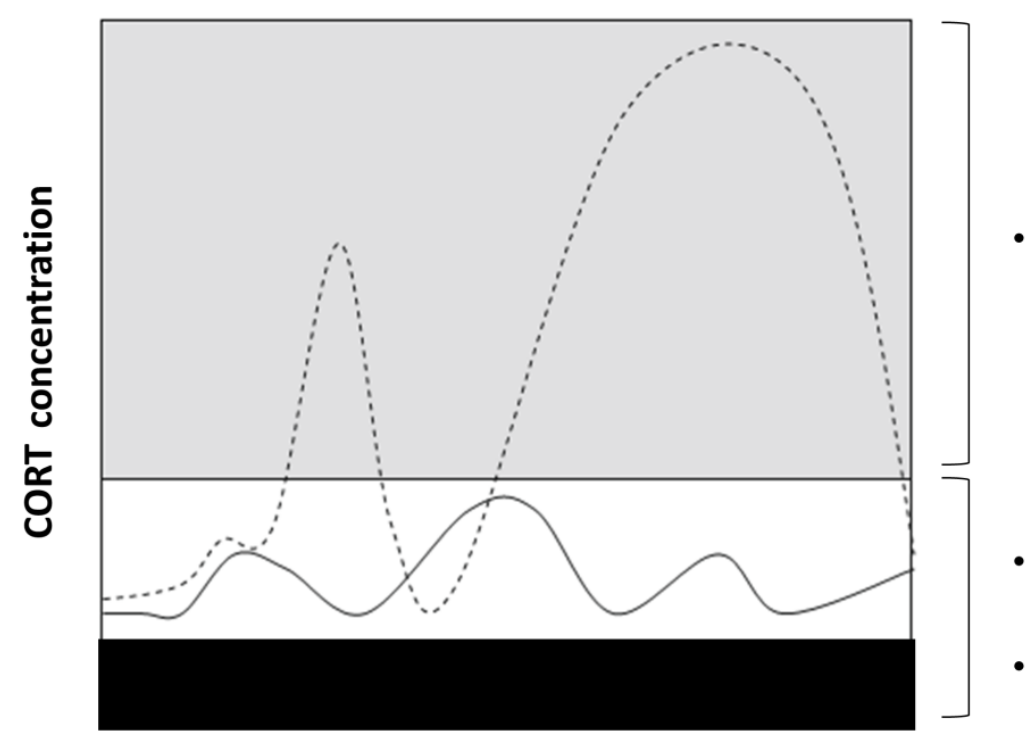

Time

\section{Acute 'CORT response'}

- physiological challenges or stressors

\section{Baseline}

- modulated: daily, seasonal, life-history cycle etc.

- necessary for glucose and water/salt regulation

Figure 1.2. Schematic diagram of CORT secretion in vertebrates. Baseline CORT concentrations reflect the basal range necessary for basic life functions (black band) and modulated range in response to daily, seasonal and life-history demands (solid black line within white band). An acute CORT response (dashed line), in which CORT concentrations are significantly elevated from baseline, will be mounted in response to physiological challenges or stressors. Figure modified from Busch and Hayward (2009).

\subsection{Corticosterone as an indicator of physiological stress}

The measurement of CORT as a tool to assess population health and/or to monitor conservation efforts is instrumental in stress physiology research, with CORT being the most widely used indicator of physiological stress in vertebrates (Busch and Hayward, 2009; Dantzer et al., 2014; Wikelski and Cooke, 2006). Increased CORT secretion in vertebrates has been linked with reduced body condition, depressed immunity, decreased locomotor performance, and changes in behaviour - all of which are performance measures that can have fitness implications (Busch and Hayward, 2009; DeNardo and Sinervo, 1994; Meylan and Clobert, 2004; Towns et al., 2007; Warner et al., 2009; Waye and Mason, 2008). 
Applied conservation physiology has been used to monitor and/or assess issues and conservation efforts such as: habitat alteration/human exposure (Owen et al., 2014; Romero and Wikelski, 2002; Taylor et al., 2014), weather and climate change (Breuner and Hahn, 2003; Lance et al., 2010; Thierry et al., 2013), increased predation and/or invasive species (Anson et al., 2013; Berger et al., 2007; Rödl et al., 2007; Thaker et al., 2010), immune function and disease (Lucas and French, 2012; Martin et al., 2012), reproduction (Klose et al., 2006; Moore et al., 2005; Smith et al., 2012; Strasser and Heath, 2013; Tartu et al., 2014), and translocations (Bosson et al., 2013; Drake et al., 2012; Franceschini et al., 2008; Holding et al., 2014a; Socorro Aguilar-Cucurachi et al., 2010; Zidon et al., 2009).

For example, Romero and Wikelski $(2001,2010)$ determined CORT secretion in Galápagos marine iguanas (Amblyrhynchus cristatus) during El Niño and found that 1) CORT secretion (both baseline CORT and the CORT response to capture-restraint) was higher during El Niño-induced famine conditions (compared to non-famine conditions), 2) baseline CORT was elevated in individuals with body condition scores below a certain threshold, and 3) the CORT response to capture-restraint and negative feedback of CORT predicted survival. Hartup et al. (2005) examined CORT secretion (faecal CORT concentrations) to assess potential stress in whooping cranes (Grus americana) undergoing translocation to the wild. The authors found that acute stressors of capture, restraint, and severe weather were connected with CORT secretion, but the overall translocation process (i.e. costume-rearing, ultralight aircraft habituation, training, artificial migration) did not lead to chronic stress.

There is still confusion, however, in how to identify chronic stress in wild animals, although it is commonly assumed that measures of CORT will increase. The best approach for identifying a chronically stressed population appears to be documentation of changes in CORT secretion, as the detection of a change in response may be more important than the direction of change (increase or decrease) (Dantzer et al., 2014; Dickens and Romero, 2013).

\subsubsection{Determining CORT concentrations}

\subsubsection{Immunoassay}


Corticosterone concentrations are determined in biological samples (i.e. blood plasma/serum, saliva, urine, faeces, hair, feather, skin, milk, and albumen/yolk) mainly by either enzyme-immunoassay (EIA) or radio-immunoassay (RIA) (Sheriff et al., 2011). Both EIA and RIA techniques use competitive binding assays that are extremely sensitive in detecting CORT. Assays can be developed in-house or be purchased as commercial kits (complete with all necessary components). Both options need to be carefully validated for the species and biological sample of choice (as in Chapter Two) to ensure proper quantification of CORT concentrations, as other variables can interfere with the assay and the validation process will identify any issues (Sheriff et al., 2011).

\subsubsection{Total vs free CORT in blood plasma samples}

Determining CORT concentrations in blood samples (by assaying plasma or serum) is the most commonly used method in vertebrate studies. Blood samples provide an instantaneous 'snap-shot' of circulating CORT concentrations in an individual, for both baseline and CORT response measures (Sheriff et al., 2011) (Fig 1.2). Assays of plasma/serum CORT measure the total available CORT concentration (versus free CORT concentration - unbound to CBGs) in a blood sample. Breuner et al. (2013) recommended that researchers determine and report both total CORT and free CORT concentrations, arguing that free CORT is the biologically relevant component in blood. However, total CORT concentrations are currently considered more accurate and more interpretable/relevant for ecological field studies than free CORT concentrations, as total CORT indicates the total available CORT that will eventually be utilised by tissues (Schoech et al., 2013).

\subsection{Tuatara ecology}

The Tuatara (Sphenodon punctatus) (Fig 1.3) is a protected reptile species endemic to New Zealand and the sole living representative of the order Rhynchocephalia, the sister group to squamates (Cree, 2014). Tuatara are sexually dimorphic once they reach sexual maturity at approximately 13 years of age $(\sim \mathrm{SVL} \geq 170 \mathrm{~mm})$, whereupon sex can be identified by examining characteristics such as head size, 
head shape, body shape, crest development and spine shape (Cree, 2014; Cree et al., 1991a; Dawbin, 1982). Tuatara have seasonally distinct breeding and nesting seasons, with breeding activity (mating and ovulation) occurring in autumn (February - March) and nesting activity occurring in spring (October - December), though not all females ovulate and nest each year (Cree, 2014). Female tuatara exhibit an extended reproductive cycle, carrying eggs in the oviduct for 6-8 months and producing one clutch of eggs every 2-9 years, depending on the population (Cree et al., 1992; Moore et al., 2009a; Refsnider et al., 2010).

Historically, tuatara were widely distributed throughout New Zealand, but following human settlement, they became extinct on the mainland (North and South Islands). Currently, natural populations occur on 32 isolated offshore islands and translocated populations occur on nine offshore islands and five mainland sanctuaries (Cree, 2014; Jones and Cree, 2012). Captive breeding programs, eradication of invasive species/predator control, and translocations (Cree, 2014; Gaze, 2001) are relevant ongoing conservation efforts for tuatara, all of which have potential to benefit from relevant endocrine data.

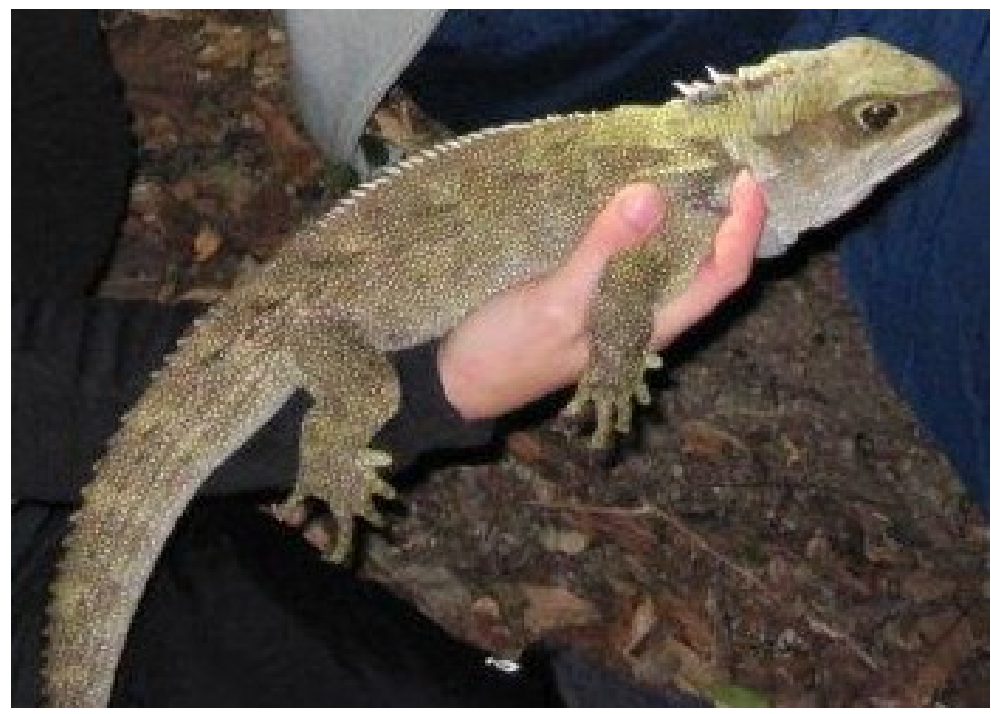

Figure 1.3. A wild adult male tuatara (Sphenodon punctatus) that was hand-captured on Taranga Island, and subsequently sampled by Lindsay Anderson. Photo: Susan Keall. 
Previous research has shown that baseline CORT in tuatara is generally low, with no evidence of a diel cycle (Cree and Tyrrell, 2001; Tyrrell and Cree, 1998), and a significant CORT response is observed to capture-restraint (Tyrrell and Cree, 1998). Seasonal variation in baseline CORT has been observed in the population on Stephens Island/Takapourewa, where baseline CORT was highest in November for females and February and May for males (Tyrrell and Cree, 1998); however, seasonal patterns of baseline CORT secretion in other island populations, seasonal patterns of the CORT response, and factors influencing inter-island variation have not been investigated. Overall, we know very little about how intrinsic and extrinsic factors influence variation in CORT secretion (both baseline CORT and the CORT response) within and among populations of tuatara.

\subsection{Study populations/sites}

The study populations/sites (Fig 1.4) in this thesis were chosen based on population size and connection to ongoing conservation efforts. Stephens Island has the largest population of tuatara, and was chosen as the study site to thoroughly examine intrinsic factors influencing CORT secretion (baseline CORT and a $24 \mathrm{~h} \mathrm{CORT}$ response time-series) in male and female (both gravid and non-gravid) tuatara (Chapter Two). The Stephens Island, North Brother Island, Lady Alice Island, and Taranga Island populations were included in studies examining the influence of temperature on CORT secretion (Chapter Three) and inter-island variation of CORT secretion (Chapter Four). Finally, Stephens Island and Lady Alice Island were source populations in two translocation programmes (Chapter Five), where I examined CORT secretion through the translocation process in the translocated populations of Motuihe Island (from Lady Alice Island), Sanctuary Mountain Maungatautari and Cape Kidnappers Sanctuary (both from Stephens Island). 


\section{$\left\{\begin{array}{l}\text { Lady Alice Island } \\ \text { Taranga Island }\end{array}\right.$}

Motuihe Island

\section{0}

Sanctuary Mountain Maungatautari

O

Cape Kidnappers Sanctuary

$100 \mathrm{~km}$

Stephens Island/Takapourewa

North Brother Island

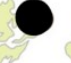
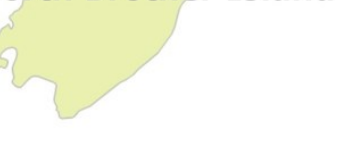

$\longleftarrow$

Figure 1.4. Location of study populations/sites mentioned in this thesis. Naturally occurring island populations are indicated by black dots and translocated populations are indicated by white dots.

\subsection{Thesis structure}

The data chapters of this thesis are written as stand-alone manuscripts that have been submitted to or published in peer-reviewed journals. Therefore, there is inevitably some repetition, specifically in the introduction and methods sections (note: references for all chapters are combined at the end of this thesis). I led the study design, data collection, data analyses, and writing of this thesis, under the guidance and supervision of my supervisors who are listed as co-authors for all four data chapters. 
The overall aim of this thesis is to advance the understanding of CORT physiology in the tuatara (Sphenodon punctatus) and to assess the value of monitoring CORT in relevant conservation efforts. The results from this thesis will contribute to the broader field of comparative endocrinology and will provide applied results and information to conservation managers and future researchers.

The main questions of this thesis are:

1) What factors influence CORT secretion (both baseline CORT and the CORT response) in tuatara?

2) Do patterns of CORT secretion indicate a physiological response to challenges/stressors in tuatara?

3) Is the measurement of CORT secretion a valuable addition to the conservation 'tool-box' for tuatara?

Basic intrinsic patterns of CORT secretion must first be understood in order to reliably interpret and/or compare outcomes from a study employing CORT secretion as a tool. Failing to account for influential factors and correlates of CORT secretion can lead to uncertain results. Therefore, in Chapter Two, I conduct a $24 \mathrm{~h}$ time-series of capture-restraint in a wild population of tuatara on Stephens Island. I simultaneously examine patterns of CORT secretion in males, non-gravid females, and gravid females. I test the hypothesis that gravid females have a dampened CORT response to capture-restraint compared to non-gravid females. I also consider correlations with body condition and internal body temperature. Chapter Two has been published as:

Anderson L, Cree A, Towns D, Nelson N (2014) Modulation of corticosterone secretion in tuatara (Sphenodon punctatus): evidence of a dampened stress response in gravid females. General and Comparative Endocrinology 201: 45-52 
Chapter Three expands on results from Chapter Two, to further examine (both observationally and experimentally) the correlation between internal body temperature $\left(T_{\mathrm{b}}\right)$ and CORT secretion. I test the hypothesis that an acute increase in $T_{\mathrm{b}}$ increases the CORT response to capture-restraint. Chapter Three has been submitted to Physiological and Biochemical Zoology as:

Anderson L, Nelson N, Cree A (in review) Increased body temperature amplifies the corticosterone stress response in tuatara (Sphenodon punctatus). Physiological and Biochemical Zoology

Taken together, Chapters Two and Three identify key intrinsic factors that are correlated with baseline CORT and the CORT response in tuatara. Chapters Four and Five build on (and consider) results from the previous chapters to examine if patterns of CORT secretion indicate a physiological response to challenges/stressors and assess the value of CORT measurement as a conservation physiology tool in tuatara conservation efforts.

Chapter Four compares CORT secretion among four contrasting island populations (with respect to potential challenges/stressors) and identifies ecological attributes that best explain variation in CORT secretion among populations. I test the hypotheses that (i) baseline CORT varies among populations, (ii) baseline CORT is higher during the breeding season, compared to the non-breeding season, and (iii) the CORT response is higher in northern populations that experience a milder climate and past/recent presence of an introduced mammal, the Pacific rat (Rattus exulans). Chapter Four has been submitted to Animal Conservation as:

Anderson L, Nelson N, Towns D, Cree A (in review) The corticosterone stress response varies among island populations of tuatara (Sphenodon punctatus) and is predicted by linear ecological attributes. Animal Conservation.

Chapter Five monitors the CORT response through the initial translocation procedure in tuatara, and examines long-term patterns of CORT secretion in 
translocated populations as an indicator of chronic stress. I test the hypothesis that cumulative stressors (experienced during the translocation process) and translocation to a novel location increase CORT secretion. Chapter Five has been published as:

Anderson L, Cree A, Towns D, Nelson (2015) Moving house: Longterm dynamics of corticosterone secretion are unaltered in translocated populations of a rare reptile (the tuatara, Sphenodon punctatus). Conservation Physiology. DOI: 10.1093/conphys/cov014

Chapter Six summarises the main findings of this thesis, discusses conservation applications and the value of measuring CORT secretion as a conservation physiology tool in tuatara, and identifies knowledge gaps and areas for future research. 


\section{Modulation of corticosterone secretion in tuatara (Sphenodon punctatus): Evidence of a dampened stress response in gravid females ${ }^{1}$}

\subsection{Abstract}

Baseline and stress response glucocorticoid (GC) secretion can be modulated by individuals to support activities and physiological functions connected with reproduction (migration, mating, oviposition and/or parturition, care of young). Corticosterone (CORT) is the primary GC in reptiles and, in accordance with other vertebrates, an adrenocortical stress response is observed. Modulation of CORT secretion occurs in several reptile species, such that elevated baseline CORT and/or a dampened CORT response are common during reproductive life-history events. I investigated CORT secretion after $24 \mathrm{~h}$ capture-restraint in the oviparous tuatara (Sphenodon punctatus), the last living rhynchocephalian, and tested whether gravid females have a dampened CORT response compared with non-gravid females. I also included males as a comparison. I confirmed that gravid females have significantly higher baseline plasma CORT than non-gravid females, suggesting increased CORT secretion during nesting. Furthermore, I found that gravid females exhibit a dampened CORT response compared to non-gravid females and males. My results demonstrate that female reproductive condition is correlated with CORT secretion in tuatara, and suggest that CORT secretion is modulated in gravid females during

\footnotetext{
${ }^{1}$ This chapter is based on the following publication with minor modifications: Anderson L, Cree A, Towns D, Nelson N (2014) Modulation of corticosterone secretion in tuatara (Sphenodon punctatus): evidence of a dampened stress response in gravid females. General and Comparative Endocrinology 201: 45-52
} 
nesting to maintain homeostasis, which could increase chances of reproductive success and/or promote overall fitness.

\subsection{Introduction}

Animals continuously adjust to their environment to support immediate survival and long-term fitness. Environmental perturbations such as increased predator abundance, decreased food availability, habitat alteration, human exposure, pollution and extreme weather can test individuals' abilities to maintain homeostasis (Angelier and Wingfield, 2013; Busch and Hayward, 2009; Langkilde and Trompeter, 2011; Romero and Wikelski, 2001; Wingfield and Sapolsky, 2003). Similarly, physiological and social demands such as reproduction, migration and territorial disputes can be challenging (Creel et al., 2013; Klukowski, 2011; Phillips and Klukowski, 2008; Yang and Wilczynski, 2003). One way that individuals manage these environmental, social and physical challenges is through release of glucocorticoids (GCs) from adrenal tissues.

GCs promote basic life processes such as energy intake and cell provisioning of glucose (Landys et al., 2006). When individuals are subject to challenging stimuli, an adaptive integrated process commonly referred to as the 'stress response' activates the hypothalamo-pituitary-adrenal (HPA) axis and leads to an increase in GC secretion (Boonstra, 2013; Busch and Hayward, 2009; Cockrem, 2013). This surge of GCs (along with other related hormones, signalling molecules and complementary processes) mobilises and diverts energy resources towards muscular, cardiovascular and cognitive systems to promote immediate survival, a reaction that is conserved across the majority of vertebrate groups (Cockrem, 2013; Sapolsky et al., 2000; Wingfield and Sapolsky, 2003). Although GCs primarily function to support immediate survival and fitness, elevated GCs over extended periods of time (i.e. chronic stress) have been shown to have deleterious effects on an individual's overall health, condition and reproductive success (Almasi et al., 2013; Sapolsky et al., 2000). Nevertheless, baseline and stress response GC secretion can be modulated by individuals to support activities and physiological functions connected 
with reproduction (migration, mating, oviposition and/or parturition, care of young).

Glucocorticoid modulation during reproductive life-history stages is attributable to higher energetic demands and serves to maintain homeostasis and promote reproductive success (Breuner et al., 2008; Love et al., 2008; Romero, 2002). Therefore, identifying and understanding modulation of GCs and the GC stress response in a context-dependent manner clarifies relationships between GC secretion, behaviour and potential fitness trade-offs (Angelier and Wingfield, 2013; Busch and Hayward, 2009; Madliger and Love, 2014).

Research investigating synthesis, secretion and modulation of GCs has focussed primarily on fish, mammals and birds, with reptiles and amphibians constituting only 10\% of studies to date (Baker et al., 2013; Bonier et al., 2009; Breuner et al., 2008; Cockrem, 2013). Reptiles are good model organisms to examine modulation of GC secretion as they exhibit a diverse range of characteristics (oviparity/viviparity, several different sex-determining patterns, various reproductive systems and life-history strategies) conducive to studying the interplay and potential trade-offs between stress, reproductive success and survival (Crews and Moore, 1986; Moore and Jessop, 2003). In reptiles, the primary GC is corticosterone (CORT), and similar to other vertebrates a CORT response to stressors is observed (Cockrem, 2013; Romero, 2002). Modulation of CORT secretion occurs in several reptile species, such that elevated baseline CORT concentration and/or a dampened CORT response are common during reproductive life-history events (Moore and Jessop, 2003; Wingfield and Sapolsky, 2003). The functional significances and underlying mechanisms of this trait in gravid/pregnant reptiles are unclear, but some authors have suggested potential links with egg/embryo maintenance (Cree and Tyrrell, 2001), with adaptive maternal effects such as shielding embryos from hormone exposure or influences on offspring phenotype (Cartledge and Jones, 2007; Uller et al., 2009; Warner et al., 2009), and with timing of oviposition/parturition (Jones and Guillette, 1982; Smith et al., 2012). 
When breeding individuals are exposed to stressors, a dampened CORT response may also serve to buffer activation of the 'emergency life-history stage ' (in which immediate survival is prioritized and activities such as mating and nesting attempts can be abandoned) to increase chances of successful reproduction (Jessop et al., 2000; Moore and Jessop, 2003; Wingfield et al., 1998; Wingfield and Sapolsky, 2003). For example, several species of sea turtle show a dampened CORT response during nesting, even when faced with additional (potentially lethal) stressors such as extreme predation attacks and overheating, suggesting a trade-off of immediate survival for an increased chance of reproductive success (Jessop et al., 2004a; Jessop et al., 2000). Lack of a significant CORT response during breeding events could also suggest an inability to mount a response due to changes in the capacity of the adrenal gland to secrete CORT (Romero, 2006).

Numerous intrinsic and extrinsic factors can influence modulation of baseline CORT concentration and the CORT response in reptiles. These include age, sex, reproductive condition, body condition, season, weather, pathogens and population (Baker et al., 2013; Boonstra, 2013; Breuner et al., 2008; Creel et al., 2013; Eikenaar et al., 2012; Wingfield, 2013). In recent reviews of the existing literature, it is apparent that failing to account for influential factors and correlates of CORT secretion can lead to uncertain results (Baker et al., 2013; Busch and Hayward, 2009; Cockrem, 2013; Creel et al., 2013; Wingfield, 2013). For example, comparing modulation of CORT secretion in breeding and non-breeding reptiles is often confounded with seasonal changes, as sampling of non-breeding individuals takes place outside of the breeding season (for both males and females) (Cartledge and Jones, 2007; Klukowski, 2011; Moore and Jessop, 2003; Selman et al., 2012). Variation between geographically separated populations (both permanently and temporarily) can also be a limiting factor. For example, in a study comparing the CORT response in breeding and non-breeding female green sea turtles (Chelonia mydas), season was controlled for but non-breeding females were part of a permanently resident reef population whereas breeding females were migratory non-residents (Jessop et al., 2000). 
To my knowledge, only two studies have simultaneously investigated the modulation of CORT secretion in reptiles, in a way that allowed for separation of the effects of sex, reproductive condition, season and population. For example, the viviparous New Zealand common gecko (Woodworthia maculatus) and oviparous tree lizard (Urosaurus ornatus) are model reptile species that allow for concurrent sampling of gravid female, non-gravid female and male individuals, without confounding factors such as population and season (Cree et al., 2003; Woodley and Moore, 2002). Interestingly, modulation of the CORT response was not observed in pregnant female common geckos; therefore, the functional significance and mechanism of the ability to modulate CORT secretion (with regards to female reproductive condition) in reptiles may not be fruitful to be explored further in this species (Cree et al., 2003). A dampened CORT response was observed in gravid female tree lizards, compared with vitellogenic females and males (Woodley and Moore, 2002). However in gravid females, an obvious temporal separation of mating, ovulation, and nesting/oviposition activity is lacking (as oviposition occurs $\sim 1$ week after ovulation), making it difficult to tease out the functional significance of CORT modulation (with regards to gravid females) in this species.

Tuatara (Sphenodon punctatus) are an attractive model species for studies investigating CORT secretion and modulation as their distribution and biology allows for simultaneous control of factors that can influence CORT release. Here, I test for a dampened CORT response in gravid females of this oviparous species during the nesting life-history stage. Tuatara are a protected reptile species endemic to New Zealand, and wild populations are currently restricted to isolated offshore islands (Jones and Cree, 2012). They are the only extant representatives of the order Rhynchocephalia, which is the sister group to squamates (Jones, 2008). Studying tuatara could therefore contribute to understanding of patterns that are general among amniotes, for example whether nesting in oviparous species is associated with a dampened CORT response to capture. Tuatara reach sexual maturity at approximately 13 years of age $(\sim \mathrm{SVL} \geq 170 \mathrm{~mm})$, and males and females can be identified by examining secondary sex characteristics such as head size/shape, body shape, crest development and spine shape (Cree et al., 1991a; Dawbin, 1982). Tuatara have a seasonally distinct reproductive cycle with mating 
taking place in the austral autumn (February - March) and nesting in the austral spring (October - November). Females do not have an annual reproductive cycle; instead, ovulation and nesting events occur only every 2-9 years (Cree et al., 1992; Moore et al., 2009a; Refsnider et al., 2010). Therefore, gravid and non-gravid females can be found in the same population at the same time, allowing for control of reproductive condition and season. Nesting is separated by 6-7 months from mating and ovulation in tuatara (Cree, 1994); therefore, patterns in CORT secretion that are related specifically to nesting/oviposition are much more readily identified than in other reptiles.

Baseline CORT secretion in tuatara is relatively low and variation is observed seasonally, but not diurnally (Tyrrell and Cree, 1998). Gravid females have significantly higher baseline CORT levels during the nesting life-history stage compared with non-gravid females and males, with levels highest during nest digging and oviposition (Cree and Tyrrell, 2001). Interestingly, a distinct fall ( $~ 5$ fold) in baseline CORT secretion is observed after oviposition, even in the case of females that continue to guard their nests, which suggests a potential role of CORT in the timing of oviposition (Cree and Tyrrell, 2001). In accordance with other vertebrate species, a stress response to capture-restraint is detected in tuatara, although CORT concentration values at $3 \mathrm{~h}$ capture-restraint, are relatively low compared to other reptiles (Tyrrell, 1993; Tyrrell and Cree, 1998). Studies examining the magnitude and duration of the CORT response are limited (Cree and Tyrrell, 2001), and CORT modulation in gravid females during the nesting lifehistory stage has not been examined.

The aim of this study was to test whether gravid females exhibit a modulated CORT response during the nesting life-history stage compared with non-gravid females. Males were included for comparison. By examining the responses of gravid females, non-gravid females and males simultaneously, I am able to identify variation in the CORT response related to sex and reproductive condition, apart from seasonal or environmental changes. I also tested for interactions between the CORT response and predictors such as body temperature and body condition, as some studies report a positive relationship between internal body temperature and baseline 
CORT concentration (including studies in tuatara) (Cree et al., 1990a; Cree et al., 2003) and a negative relationship between body condition and magnitude of the CORT response (Baker et al., 2013; Dunlap and Wingfield, 1995).

\subsection{Material and methods}

\subsubsection{Study animals and experimental design}

Wild adult tuatara (Sphenodon punctatus) were captured and sampled on Stephens Island/Takapourewa (40 $40^{\prime} \mathrm{S}, 174^{\circ} 00^{\prime} \mathrm{E}$ ) in Cook Strait, New Zealand during the 2011 nesting season (October - December, austral spring). Individuals that had emerged from burrows were caught by hand at night between 20:00 $\mathrm{h}$ and 05:00 $\mathrm{h}$ and were subsequently assigned to an appropriate group based on sex and reproductive status (male, gravid female, or non-gravid female). Gravid females were selectively captured at or near a nesting rookery, and female reproductive status was inferred through abdominal palpation for shelled eggs. Following the blood sampling described in section 2.3.2, synthetic oxytocin (10 I.U./ml/kg body mass) (Thompson et al., 1991) was administered by an intraperitoneal injection to a subset of gravid females ( $n=13$ ) (as a component of another study - unpublished). Oviposition was observed within $24 \mathrm{~h}$ in 12 of the 13 females, which helped validate the inference of reproductive status from palpation.

\subsubsection{CORT response to 24 h capture-restraint}

Male $(n=30)$, gravid female $(n=28)$ and non-gravid female $(n=31)$ tuatara were subject to a standardized capture-restraint protocol to determine patterns of CORT secretion over time. Capture/sampling occurred over 2 nights during the 2011 nesting season (25-26 October). To determine baseline CORT levels, a blood sample (up to $1 \mathrm{ml}$ ) was collected within $10 \mathrm{~min}$ of capture (mean $=5.22 \pm 0.34 \mathrm{~min}$ ) from the base of the tail with a heparinized 23-gauge needle and $1 \mathrm{ml}$ syringe. Previous studies of tuatara observed no significant effect of bleeding effort (time from capture to blood sample collection) on baseline CORT concentration in blood samples collected within 20 min of capture (Tyrrell, 1993). As expected, I observed no correlation between duration of bleeding effort and baseline CORT concentration for 
samples obtained within 10 min of capture $(r=-0.085, P=0.429)$. After baseline samples were taken, individuals were randomly assigned to capture-restraint treatment times of $1.5,3,6,12,18$ or $24 \mathrm{~h}$ ( $n=4-5 /$ group/treatment time) to determine the $24 \mathrm{~h}$ CORT response and were held in a cloth capture bag for assigned times in a quiet room inside the research house (basic accommodation on Stephens Island). A second blood sample (up to $1 \mathrm{ml}$ ) was taken from each individual immediately after the assigned capture-restraint treatment time was reached. Individuals were randomly selected to obtain desired sample sizes and were sampled for blood only twice in $24 \mathrm{~h}$. Blood samples were separated into plasma and red blood cell components under normal gravity (as centrifugation was not available at the remote island field site) for 6 to $8 \mathrm{~h}$ at $4^{\circ} \mathrm{C}$ (CORT levels in plasma and serum have been shown to remain stable when kept at room temperature and $4^{\circ} \mathrm{C}$ for up to $72 \mathrm{~h}$, with and without centrifugation)(Reimers et al., 1983; Sheriff et al., 2011). Plasma was transferred into cryogenic vials with a micropipette and held in a freezer at $-20^{\circ} \mathrm{C}$ until returned to the laboratory where it was stored at $-80^{\circ} \mathrm{C}$ until assayed.

\subsubsection{Internal body temperature and body-condition measurements}

Internal body temperature $\left(T_{\mathrm{b}}\right)$ was recorded with a cloacal thermocouple (Fluke ${ }^{\circledR}$ Multimeter, model: 179 , specified accuracy $\pm 0.1^{\circ} \mathrm{C}$, USA) prior to taking blood samples for each individual (both baseline and stress response). Morphometric measurements were taken after capture-restraint and CORT response blood sampling was completed. Individual animals were weighed to the nearest $\pm 5 \mathrm{~g}$ with a $1000 \mathrm{~g}$ spring scale (Pesola AG, Switzerland) to determine mass (g), and snoutvent length $(\mathrm{mm})$, tail length $(\mathrm{mm})$, tail regeneration length $(\mathrm{mm})$, and pelvis width $(\mathrm{mm})$ were measured with a straight ruler. I calculated tail-corrected mass following the equation described by Newman et al. (1994) to account for tail-loss and regeneration (Newman et al., 1994) . I pooled morphometric measurement data (male, non-gravid, gravid) to generate individual body-condition scores using principal components analysis (PCA) to incorporate size-index traits related to condition (SVL, width, mass) (Taillon et al., 2011). This created a correlation matrix that produced a 'size-index' scoring system in which the first principal component (PC1) explained $84.9 \%$ of the total variance. For this study, PC1 scores are 
considered to be a measure of tuatara body condition. The body-condition score (PC1) was dominated by the following morphometric loadings: tail-corrected mass (TCM), pelvis width (PW) and snout-vent length (SVL). The scoring system equation for PC1 is $0.602 \mathrm{TCM}+0.534 \mathrm{PW}+0.593 \mathrm{SVL}$, and as all loadings have positive coefficients this indicates that individuals with greater measurements of TCM, PW and SVL will have higher body-condition scores. This scoring system was compared with an alternative scoring system (standardized residuals from the regression of TCM on SVL) and a strong correlation was observed $(r=0.981, P<0.001)$.

\subsubsection{Hormone determination and enzyme immunoassay (EIA) validation}

Plasma samples were randomly selected for assay (29/plate), thawed at room temperature and spun in a centrifuge at $5000 \mathrm{rpm}$ for $5 \mathrm{~min}$ at $4^{\circ} \mathrm{C}$ to separate any residual debris. Aliquots of $10 \mu \mathrm{l}$ plasma were extracted once with $600 \mu \mathrm{l}$ of freshly distilled dichloromethane. After $15 \mathrm{~min}$ of incubation at room temperature, samples were snap-frozen in a $-70^{\circ} \mathrm{C}$ ethanol bath, decanted into clean glass vials, and dried in a vacuum oven at $37^{\circ} \mathrm{C}$ for $2 \mathrm{~h}$. Samples were reconstituted with $120 \mu \mathrm{l}$ EIA buffer (for a 12-fold dilution), vortexed and held at $4^{\circ} \mathrm{C}$ until assayed. Extraction efficiency was measured by comparing mean recovery of extracted $(n=5)$ versus non-extracted $(n=5) 2000 \mathrm{cpm}$ tritiated CORT $\left(\mathrm{H}^{3}\right)$. Mean extraction efficiency of $\mathrm{H}^{3}$ was $101 \% \pm$ $2 \%$ s.d. ( $n=9$ extractions) with an overall CV of 1.64\%. Extracted plasma samples $(50 \mu \mathrm{l})$ were analysed in duplicate using commercial enzyme immunoassay kits (Cayman Chemical Co., Ann Arbor, MI, note: kit validation for first-time use in tuatara is detailed below) containing 96-well plates coated with rabbit polyclonal anti-sheep IgG antibody. CORT-specific acetylcholinesterase (AChE) tracer and sheep CORT antiserum were added to sample wells and placed on an orbital shaker for $2 \mathrm{~h}$. Plates were washed five times, developed for $1 \mathrm{~h}$ on an orbital shaker and subsequently read at $405 \mathrm{~nm}$. The concentration of CORT was calculated by comparing results to a standard curve. Samples that did not yield CORT concentrations within the $10-90 \%$ bound range were re-assayed after an appropriate dilution.

To calculate the intra-assay co-efficient of variation (CV), three quality-control (QC) samples with binding levels of $20 \%, 50 \%$ and $65 \%$ were prepared in extracted 
tuatara plasma and analysed (in duplicate) repeatedly throughout one assay. Replicate aliquots $(n=9)$ of the QCs yielded similar plasma CORT concentrations (ng/ml) at each level, with \%CVs of 8.3, 10.4 and 13.9 for the $20 \%, 50 \%$ and $65 \%$ bound QCs, respectively. The mean intra-assay CV for all QCs was $10.9 \%$. Interassay CVs were calculated from the same QCs placed at the start and end wells (in duplicate) of each assay plate $(n=8)$. Mean inter-assay CVs were $11.4 \%$ (20\% bound QC), $12.2 \%$ (50\% bound QC), and 13.5\% (65\% bound QC). The mean inter-assay CV for all QCs was 12.4\%. Serial dilutions at 100\%, 80\%, 60\%, 40\%, 20\%, and 10\% of pooled tuatara plasma samples showed good parallelism to the standard curve for CORT over the assay standard range. Multiple linear regression analysis was used to provide confirmation that the serial dilutions were comparable to the CORT standard curve provided $(P=0.746)$. Linear regression equations were: $\% \mathrm{~B} / \mathrm{B}_{0}=$ $48.9-42.3 \log \operatorname{cort}\left(r^{2}=99.2, P<0.001\right)$ for the serial dilution and: $\% \mathrm{~B} / \mathrm{B}_{0}=5.6-40.7$ logcort for the standard curve $\left(r^{2}=99.1, P<0.001\right)$. To determine the minimum detectable concentration (MDC) of CORT I assayed $\mathrm{B}_{0}$ wells ( $n=16$, each in duplicate) on a single plate and calculated mean CORT concentration minus two standard deviations. Results showed a MDC of $0.03 \mathrm{ng} / \mathrm{ml}$, which corresponds with the MDC supplied by the EIA kit manufacturers.

\subsubsection{Statistical analyses}

Data analyses were carried out using R v3.0.0 statistical software (R Development Core 2008) and Prism 6 (Graphpad Software Inc.). All data were checked for normal distributions and homoscedasticity, and if necessary, were transformed to meet assumptions for parametric statistical tests. A linear mixed effects regression (LMER) model was fitted using the 'Ime4' (Bates, 2013) package in R to investigate the influence of sex, reproductive condition, $T_{\mathrm{b}}$, body condition and duration of capture-restraint $(0-24 \mathrm{~h})$ on the CORT response. Log-transformed CORT was the response variable, predictors were group (male, gravid female, non-gravid female), duration of capture-restraint (h), $T_{\mathrm{b}}$ and body condition were fixed main effects (with significant interaction terms included), and tuatara ID was included as a random effect to account for repeat sampling of individuals for baseline CORT and CORT response values. The 'languageR' package (Baayen, 2011) was used to compute $P$-values based on Markov-chain Monte Carlo (MCMC) sampling. 
Significance for all tests was assumed at $p<0.05$. To quantify the magnitude of the mean CORT response (ng/ml) over the 24 h capture-restraint period, I calculated the area under the curve (AUC) in Prism 6 using the trapezoid rule, where AUC values reflect an 'integrated CORT response' for each group (Cockrem and Silverin, 2002; Narayan et al., 2011a). Baseline values were set at zero and negative values were not included. I used linear models (LMs) and post-hoc contrast tests to compare body condition between groups, and to investigate the relationship between $T_{\mathrm{b}}$ and baseline CORT secretion in all groups. I used a LMER model to compare $T_{\mathrm{b}}$ between groups and sample times (at baseline and CORT response sampling), with tuatara ID included as a random effect to account for repeat $T_{\mathrm{b}}$ sampling of individuals.

\subsection{Results}

\subsubsection{CORT response to 24 h capture-restraint}

I analysed the effect of acute $24 \mathrm{~h}$ capture-restraint on CORT secretion profiles in gravid, non-gravid and male tuatara by fitting a linear mixed effects regression (LMER) model to CORT concentrations from 0 to 24 hours. Neither $T_{\mathrm{b}}$ nor body condition were significant predictors of the CORT response across groups and were therefore excluded from the final model (Table 2.1). Mean baseline CORT concentrations $(\mathrm{ng} / \mathrm{ml})$ were significantly higher in gravid females $(5.75 \pm 1.21)$ compared to non-gravid females (1.05 \pm 0.15$)$ and males (1.30 \pm 0.19$)$ (Fig 2.1a, Table 2.1). The CORT response over $24 \mathrm{~h}$ was significantly dampened in gravid females compared with non-gravid females and males (Fig 2.1b-d, Table 2.1). By determining AUCs, I was able to quantify the magnitude of the mean CORT response (ng/ml) over a $24 \mathrm{~h}$ period of capture-restraint stress (Fig 2.1b-d). Gravid females had the lowest mean integrated CORT response $(\mathrm{ng} / \mathrm{ml} / 24 \mathrm{~h})$ at 2.61 , followed by males at 11.13 (4-fold greater than gravid females) and non-gravid females at 13.36 (5-fold greater than gravid females). Peak secretion of CORT occurred at 1.5 hours for gravid females, and at 12 hours for both non-gravid females and males (Fig 2.1bd). 
Table 2.1: Results from linear mixed effects regression models explaining variation in measures of baseline and stress response corticosterone secretion $(\mathrm{ng} / \mathrm{ml})$ as a function of time $(0-24 \mathrm{~h})$ after explaining variation accounted for by the fixed effects of sex, reproductive condition, duration of capture-restraint and the interaction of these effects ${ }^{1}$.

\begin{tabular}{lcccc}
\hline Fixed effect predictor & Est. & $\begin{array}{c}\text { Lower } \\
\mathbf{9 5 \% C I}\end{array}$ & $\begin{array}{c}\text { Upper } \\
\mathbf{9 5 \% C I}\end{array}$ & $\begin{array}{c}\boldsymbol{P} \text { - } \\
\text { MCMC }\end{array}$ \\
& & & & \\
gravid females (Intercept) & 0.792 & 0.673 & 0.910 & $<0.001$ \\
non-gravid females & -0.626 & -0.788 & -0.462 & $<0.001$ \\
males & -0.656 & -0.825 & -0.495 & $<0.001$ \\
capture-restraint (gravid females) & -0.002 & -0.014 & 0.010 & 0.718 \\
capture-restraint $\times$ non-gravid females & 0.017 & 0.001 & 0.035 & 0.041 \\
capture-restraint $\times$ males & 0.023 & 0.007 & 0.041 & 0.011 \\
& & & & \\
\hline
\end{tabular}

\footnotetext{
${ }^{1}$ Coefficient estimates (positive or negative) are shown and indicate direction of the linear regression from the specified intercept. 95\% credible intervals $(\mathrm{Cl})$ are shown. $P$-values based on MCMC sampling are shown.
}

\subsubsection{Relationships with body temperature and body condition}

Neither $T_{\mathrm{b}}(P>0.05)$ nor body condition $(P>0.05)$ were significant predictors for the CORT response. I found a significant positive relationship between $T_{\mathrm{b}}$ and baseline CORT concentrations $(\mathrm{ng} / \mathrm{ml})$ in gravid females only $(\mathrm{LM}, \log \mathrm{CORT}=-0.603+0.108$ $T_{\mathrm{b}}, r^{2}=0.210, P=0.015$, Fig 2.2). $T_{\mathrm{b}}$ was comparable across groups at baseline $\left(F_{2,86}=1.147, P=0.322\right)$ and during CORT response $\left(F_{2,86}=0.128, P=0.880\right)$ sampling. An increase in $T_{\mathrm{b}}$ from baseline to CORT response sampling occurred in all groups (LMER, $P<0.001$ ). Mean $T_{\mathrm{b}}\left({ }^{\circ} \mathrm{C}\right)$ at baseline sampling was $12.2 \pm 0.4$ for gravid females, $11.7 \pm 0.3$ for non-gravid females and $11.4 \pm 0.4$ for males, and at CORT response sampling was $13.8 \pm 0.3$ for gravid females, $13.9 \pm 0.2$ for non-gravid females and $13.7 \pm 0.2$ for males. On average, males were larger in body size, but I did not find a significant difference in body condition scores across groups $\left(F_{2,86}=1.374, P=0.259\right)$. 
(a) baseline $0 \mathrm{~h}$
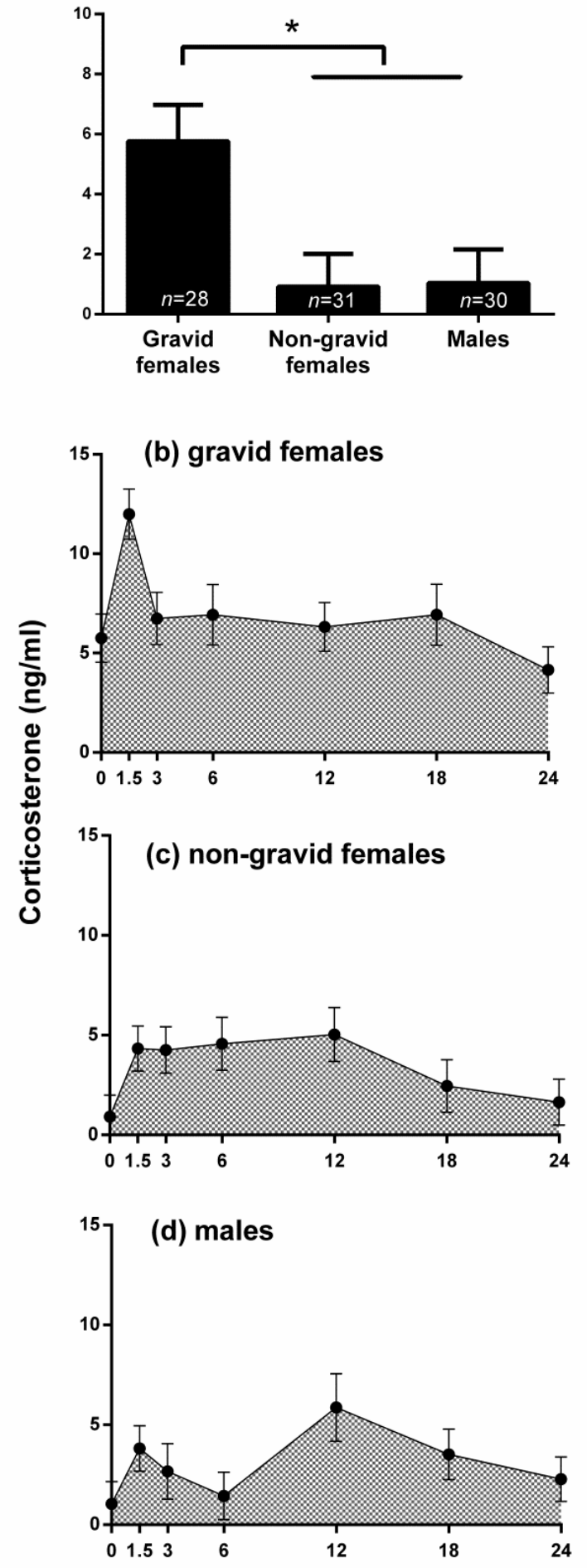

Duration of capture-restraint $(\mathrm{h})$

Figure 2.1: Patterns in (a) baseline CORT concentration $(\mathrm{ng} / \mathrm{ml}$ ) and the $24 \mathrm{~h}$ CORT response in (b) gravid female, (c) non-gravid female and (d) male tuatara (Sphenodon punctatus) over a period of $24 \mathrm{~h}$ capture-restraint. Data points represent means $\pm \mathrm{SE}$. Baseline CORT concentrations are also indicated by dashed lines in (b)-(d). Shaded curves delineate the integrated CORT response $(\mathrm{ng} / \mathrm{ml} / 24 \mathrm{~h}$ ) for each group calculated by area under the curve (AUC). 


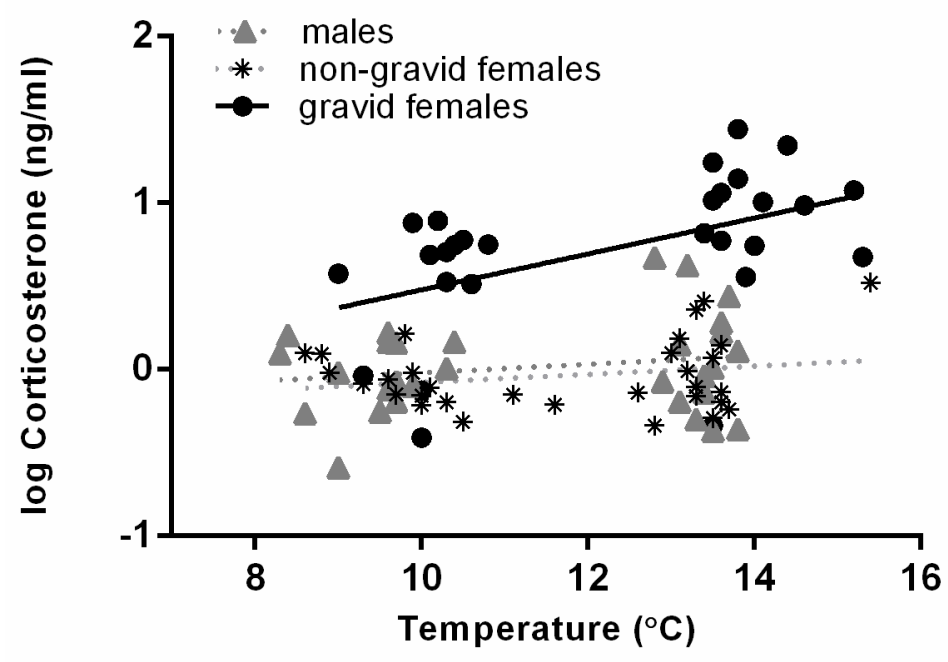

Figure 2.2: Relationship between cloacal body temperature $\left({ }^{\circ} \mathrm{C}\right)$ and the log of baseline CORT concentration $(\mathrm{ng} / \mathrm{ml})$ in male (grey dotted line $\mathrm{w} /$ triangles), non-gravid female (black dotted line $\mathrm{w} / \mathrm{stars}$ ) and gravid female (black solid line $\mathrm{w} / \mathrm{circles}$ ) tuatara (Sphenodon punctatus). A significant relationship was observed in gravid females only $\left(r^{2}=0.210, P=0.015\right)$.

\subsection{Discussion}

As expected, gravid females had significantly higher baseline CORT concentrations compared with non-gravid females (and males) during the nesting life-history stage. This result is consistent with a previous study on wild tuatara, in which the mean plasma concentration of baseline CORT in gravid females was almost twice that of non-gravid females and males (Tyrrell and Cree, 1998). Here, I established that the CORT response is dampened (4- to 5-fold) in gravid females, compared with nongravid females and males during $24 \mathrm{~h}$ capture-restraint. A dampened CORT response has been observed in other oviparous reptiles during the gravid lifehistory stage, including female tree lizards (Woodley and Moore, 2002) green turtles (Jessop et al., 2000) and olive ridley turtles (Lepidochelys olivacea) (Valverde et al., 1999). This naturally leads to the question of why some gravid oviparous reptile species modulate the CORT response (specifically down-regulating CORT secretion) during a physiologically and behaviourally challenging situation (Jessop, 2001; Moore and Jessop, 2003). Possible explanations for this trait include: adaptive 
interruption of certain stress response reactions (e.g. flight response) to nest successfully, adaptive maternal effects and/or programming, lack of awareness towards certain stressors, or an inability to mount a further CORT response. Furthermore, alternative physiological mechanisms such as changes in binding proteins (CBGs) and/or CORT receptors at target tissues may play a role in the CORT response to stress in gravid individuals (Romero, 2002).

Gravid reptiles that are actively nesting could have a suppressed CORT response to interrupt or shield activation of the emergency life-history stage (in which selfmaintenance takes precedence) in order to successfully carry out nesting (Wingfield et al., 1998). Suppression of the CORT response during nesting has been observed in other amniotes, including reptiles, and may serve to increase reproductive success and overall fitness. Female sea turtles show a dampened CORT response during nesting activities, and maintain suppression of a CORT response in the face of predation attacks (Jessop et al., 2004a), thermal stress (Jessop et al., 2000) and overcrowding (Jessop and Hamann, 2004) in order to successfully nest. Similarly, studies in birds report modulation of the CORT response in order to increase reproductive success (Lendvai et al., 2007; Wingfield et al., 1994; Wingfield and Sapolsky, 2003), with a few authors noting increased CORT secretion that leads to nest abandonment (Spee et al., 2010; Strasser and Heath, 2013; Thierry et al., 2013).

An attenuated CORT response could also be a product of adaptive maternal effects such as protecting eggs/embryos from potential deleterious effects of hormones, or allowing maternal programming of offspring to occur. Maternal hormones have been discovered in yolk of numerous vertebrate species, including birds (Almasi et al., 2012; Hayward and Wingfield, 2004), fish (Manire et al., 2004) and reptiles (Rhen et al., 2006). Increased levels of maternal CORT can affect exposure of developing embryos to CORT (Cree et al., 2003; Uller et al., 2009), which can influence phenotype, behaviour, and fitness performance measures. For example, maternal sources of CORT can influence offspring size and sex-ratio (Uller et al., 2009; Warner et al., 2009). Pregnant females with increased plasma CORT concentrations produced offspring that exhibited decreased anti-predator behaviour in garter snakes (Thamnophis elegans) (Robert et al., 2009) and decreased activity, 
sprint speed, and motivation to run in common lizard hatchlings (Lacerta vivipara) (Belliure et al., 2004; Meylan and Clobert, 2004). Future studies could investigate and possibly manipulate maternal transfer of CORT to eggs of tuatara to further explore the potential relationship with yolk hormones and subsequent offspring development, phenotype and performance.

It is also possible that gravid females that lack a stress response to capture-restraint (or in which the stress response is attenuated) do not actually perceive the stressor as a threat during specific life-history events such as nesting, oviposition, or parturition. Therefore, behavioural resistance to stressors could effectively modulate CORT secretion to maintain reproductive behaviour (Jessop et al., 2004a; Jessop et al., 2000; Jessop et al., 1999b). Threat perception could be tested in tuatara by intensifying or compounding the stressor (e.g. heat/cold-stress or exposure to predators in addition to capture-restraint stress) to see if there is a behavioural threshold that activates the CORT response. Finally, the absence of a significant CORT response in gravid females could be attributed to the fact that gravid females are operating at maximal CORT secretion during nesting or are experiencing a form of chronic stress, and are simply unable to further mount a response. This could be tested further by administering an ACTH challenge (Cartledge and Jones, 2007; Preest et al., 2005) (addressed in Appendix A).

The functional significance of elevated baseline CORT in gravid oviparous reptiles (and the potential relationship with oviposition) has received little attention, even though several oviparous reptile species exhibit elevated concentrations of baseline CORT concentrations directly preceding oviposition (Cree and Tyrrell, 2001; Jessop, 2001; Moore and Jessop, 2003). Female tuatara have a unique egg development/maintenance strategy, retaining their eggs for the longest known period in reptiles (7-9 months) (Cree et al., 1992). Therefore, elevated baseline CORT concentrations during the nesting life-history stage in tuatara cannot be attributed to sexual receptivity or ovulation, as these mating events are greatly separated in time from nesting. Prior studies on tuatara (Cree and Tyrrell, 2001) and marine iguanas (Rubenstein and Wikelski, 2005) show that baseline CORT concentrations decline shortly after oviposition, which suggests a possible role in 
the timing of egg-laying, and other studies suggest hormonal control of ovulation and parturition in viviparous and oviparous reptiles (Jones and Guillette, 1982). For example, it was shown that embryonic production of CORT may trigger parturition in viviparous southern snow skinks (Niveoscincus microlepidotus) (Girling and Jones, 2006), and exogenous elevation of baseline CORT concentrations in gravid eastern three-lined skinks (Bassiana duperreyi) induced 'premature' oviposition (Radder et al., 2008). These findings suggest that up-regulating baseline CORT secretion during nesting could function to stimulate oviposition in gravid reptiles. This could be investigated further by experimentally manipulating baseline CORT secretion in gravid females to see if there is a relationship between CORT secretion and oviposition (Appendix A).

In the current study, neither $T_{\mathrm{b}}$ nor body condition were significant predictors of the CORT response in tuatara. However, I found a significant positive relationship between $T_{\mathrm{b}}$ and baseline CORT secretion in gravid females only, with no relationship observed between $T_{\mathrm{b}}$ and the CORT response in non-gravid females or males. An increase in $T_{\mathrm{b}}$ occurred between capture and CORT response sampling; however, this is most likely due to sheltered conditions (in the capture bags), rather than a physiological response (Tyrrell and Cree, 1998). As individuals in this study were captured and sampled over two nights, $T_{\mathrm{b}}$ varied over a limited range $\left(8^{\circ} \mathrm{C}-15^{\circ} \mathrm{C}\right)$ and approached the lower end of field $T_{\mathrm{b}}$ observed in tuatara (emergence and activity occurs between $\sim 6^{\circ} \mathrm{C}$ and $25^{\circ} \mathrm{C}$ (Besson and Cree, 2010; Saint Girons et al., 1980) ); therefore, it would be useful to investigate CORT secretion at increased $T_{\mathrm{b}}$ over a wider range (see Chapter Three). Likewise, I did not find a relationship between body condition and baseline CORT secretion or the CORT response in females (gravid and non-gravid) and males. The influence of body condition on CORT secretion is quite variable among reptile species, with some studies reporting a significant influence (Baker et al., 2013; Dayger et al., 2013; Dunlap and Wingfield, 1995; Hews and Baniki, 2013; Jessop et al., 2004c; Waye and Mason, 2008), no relationship (Baker et al., 2013; Jessop et al., 2004c; Selman et al., 2012) or both (Wikelski and Romero, 2003). Interestingly, Wilkelski and Romero (2003) found that CORT secretion is elevated in marine iguanas (Amblyrhynchus cristatus) that have body condition scores below a critical threshold level, but they did not find a 
significant correlation between CORT and body condition in individuals with scores above the threshold level. Studies incorporating an expanded range of body condition scores (including populations in obvious poor condition) could clarify the relationship between body condition and CORT secretion in tuatara, and may identify a critical threshold level (related to body condition) at which CORT secretion is affected. Nonetheless, the influence of $T_{\mathrm{b}}$ and body condition (and possible physiological thresholds of both measurements) on CORT secretion are areas of research that require further study in reptiles.

\subsection{Conclusions}

I found that the CORT response is dampened in gravid female tuatara during the nesting life-history stage, which suggests modulation of CORT secretion to support nesting success. Perhaps down-regulating CORT secretion during exposure to acute stressors facilitates reproductive success by ensuring that nesting activities carry on regardless and that eggs/embryos are potentially shielded from elevated levels of maternal CORT. I confirmed that female reproductive condition is significantly correlated with baseline CORT secretion, with gravid females showing levels that are significantly higher than non-gravid females (and also males). The functional significance of elevated baseline CORT secretion in gravid females during nesting is unclear; therefore, it would be useful to carry out experimental studies involving hormone manipulation (in which CORT secretion is increased or decreased) in gravid females (see Appendix A). This would provide insight into whether CORT is being secreted at maximal levels during nesting, if/how it plays a role in oviposition and nesting behaviour, and how CORT modulation contributes to reproductive success and overall fitness in tuatara, the sole extant representative of an ancient order of reptiles. 


\section{Body temperature is correlated with the corticosterone stress response in tuatara (Sphenodon punctatus) ${ }^{1}$}

\subsection{Abstract}

When vertebrates experience environmental, physiological or social challenges, the glucocorticoid (GC) stress response is activated and rapid secretion of GCs ensues as an essential life process to promote survival. Ambient temperature affects essential life processes in most organisms. In reptiles, ambient temperature directly influences internal body temperature $\left(T_{\mathrm{b}}\right)$, which has downstream effects on physiology and behaviour. In a natural setting, fluctuations in temperature occur routinely, yet despite its relevance to essential processes in reptiles, $T_{\mathrm{b}}$ has received limited attention with regards to its influence on the GC stress response. In this study, I examined the influence of $T_{\mathrm{b}}$ on baseline secretion of corticosterone (CORT), the main GC in reptiles, and on the CORT response to capture-restraint across a range of $T_{\mathrm{b}}$ in free-living tuatara (Sphenodon punctatus) (males, non-gravid females and gravid females) I also tested the effect of an acute increase in $T_{\mathrm{b}}$ on the magnitude of the CORT response. I confirmed a positive correlation between $T_{\mathrm{b}}$ and the CORT response to capture in all groups, and a positive correlation between $T_{\mathrm{b}}$ and baseline CORT in gravid females only. Furthermore, gravid females only exhibited a significant CORT response to capture at high $T_{\mathrm{b}}$, which in combination with results from Chapter Two, suggests a response threshold at $\sim 22-25^{\circ} \mathrm{C}$ (i.e. at or

\footnotetext{
${ }^{1}$ This chapter is based on the following manuscript submitted to Physiological and Biochemical Zoology (in review) with minor modifications: Anderson L, Nelson N, Cree A. Increased body temperature amplifies the corticosterone stress response in tuatara (Sphenodon punctatus).
} 
above selected temperature). Overall, this study confirms experimentally that $T_{\mathrm{b}}$ is correlated with CORT secretion in a cold-adapted reptile and should be considered in studies on other ectotherms, especially when fluctuations in $T_{\mathrm{b}}$ are experienced.

\subsection{Introduction}

Free-living organisms are regularly exposed to environmental fluctuations. To cope with daily, seasonal and annual variation in environmental conditions, individuals adjust their physiology and behaviour to maintain essential processes and optimise fitness. One way that vertebrates adjust to environmental conditions is through secretion of glucocorticoids (GCs) from adrenal tissues. GC secretion upholds everyday maintenance such as energy intake and cell provisioning of glucose (Landys et al., 2006), and variation in GC secretion (ranging from hourly to annually) is observed within and between species. Furthermore, when individuals experience environmental perturbations, the hypothalamo-pituitary-adrenal (HPA) axis is activated and GC secretion is rapidly increased to promote immediate survival (Boonstra, 2013; Busch and Hayward, 2009; Cockrem, 2013). Although numerous studies have investigated factors that affect GC secretion (both baseline and in response to stressors) (Baker et al., 2013; Boonstra, 2013; Breuner et al., 2008; Creel et al., 2013; Wingfield, 2013), few studies consider the influence of body temperature $\left(T_{\mathrm{b}}\right)$.

Ambient temperature affects essential life processes in most organisms. In ectotherms, most notably reptiles, ambient environmental temperature directly influences $T_{\mathrm{b}}$, which has downstream effects on several aspects of physiology and behaviour (Adams et al., 1989; Firth et al., 1989; Jasnic et al., 2013; Seebacher et al., 1999). Despite its relevance to physiological processes in reptiles, $T_{\mathrm{b}}$ has received limited consideration in the scientific literature with regards to GC secretion. In reptiles, corticosterone (CORT) is the primary GC in reptiles, and as in other vertebrates, an adrenocortical stress response is observed (Cockrem, 2013; Romero, 2002). Numerous intrinsic and extrinsic factors are associated with CORT secretion in reptiles. Examples include sex, reproductive condition, body condition, disease, population, season and pathogens (Baker et al., 2013; Barry et al., 2010; Berger et al., 
2005; Eikenaar et al., 2012; Selman et al., 2012). However, the relationship between $T_{\mathrm{b}}$ and baseline CORT secretion in reptiles remains unclear. Some studies suggest a relationship while others question it, and the direction and magnitude of the relationship (if any) varies within and between species (Baker et al., 2013; Cree et al., 2003; Dupoue et al., 2013; Li et al., 2011; Sykes and Klukowski, 2009; Tyrrell and Cree, 1998). Among the limited studies that exist, most have focussed on the influence of $T_{\mathrm{b}}$ on baseline CORT secretion. To my knowledge, only two studies have experimentally tested the influence of $T_{\mathrm{b}}$ on the CORT response in reptiles.

Furthermore, the species used in these studies were both warm-adapted snakes that were exposed to a either a sub-optimal temperature treatment in captivity (Dupoue et al., 2013) or to $1 \mathrm{~h}$ acute temperature treatments (semi-aquatic snakes were warmed or cooled within physiological limits) (Sykes and Klukowski, 2009). These studies do not clarify the effect of $T_{\mathrm{b}}$ on the CORT response in free-living terrestrial ectotherms. Moreover, neither study considers the association between $T_{\mathrm{b}}$ and CORT secretion (baseline CORT and the CORT response) in gravid females.

Tuatara (Sphenodon punctatus) are a cool-climate terrestrial reptile with a low mean preferred body temperature $\left(T_{\text {sel }}\right)$ of about $21^{\circ} \mathrm{C}$ in laboratory conditions (Besson and Cree, 2010), although $T_{\mathrm{b}}$ in basking individuals (in both laboratory and field conditions) can reach $25-30^{\circ} \mathrm{C}$ (Barwick, 1982; Besson and Cree, 2010; Cree, 2014; Saint-Girons et al., 1981; Stebbins, 1958). Hence, tuatara provide a valuable opportunity to examine the influence of $T_{\mathrm{b}}$ (at capture in natural conditions and in warmer increments up to $T_{\text {sel }}$ ) on CORT secretion in a cold-adapted ectotherm. Here, I conduct an observational field study to examine baseline CORT secretion and the CORT response across a range of ambient environmental temperatures, and experimentally test whether elevated $T_{\mathrm{b}}$ during capture-restraint stress affects the CORT response.

Tuatara are protected reptile species endemic to New Zealand and natural populations are currently restricted to isolated offshore islands (Jones and Cree, 2012). They are the only extant representatives of the order Rhynchocephalia, the sister group to squamates (Jones, 2008). Tuatara have a seasonally distinct reproductive cycle with mating taking place in the austral autumn (February - 
March) and nesting in the austral spring (October - November). Individual females do not reproduce annually; instead, ovulation and nesting events occur only every 25 years (Cree et al., 1992; Moore et al., 2009a; Refsnider et al., 2010). Therefore, males and non-gravid females can be found in the same population at the same time (along with gravid females in spring), which allows for control of sex, reproductive condition, population and season.

Baseline CORT secretion in tuatara is relatively low and variation is observed seasonally, but not diurnally (Tyrrell and Cree, 1998), and a CORT response to capture-restraint stress is detected (Tyrrell, 1993; Tyrrell and Cree, 1998; Chapter Two). A previous study has reported observations of a positive relationship between $T_{\mathrm{b}}$ and baseline CORT secretion in males (but not females) during summer and winter (Tyrrell and Cree, 1998). More recently, a positive relationship between $T_{\mathrm{b}}$ and baseline CORT secretion was observed in gravid females (but not males or females) during spring, whereas no association was observed between $T_{\mathrm{b}}$ and the CORT response (Chapter Two); however, the range of $T_{\mathrm{b}}\left(8-15^{\circ} \mathrm{C}\right)$ observed in that study was narrow and at the lower end of $T_{\mathrm{b}}$ observed in natural populations of tuatara.

There were two main aims to the present study. First, I examined whether $T_{\mathrm{b}}$ is associated with baseline CORT secretion and the magnitude of the CORT response in natural populations of tuatara. Second, I experimentally tested whether an acute increase in temperature influenced the magnitude of the CORT response in males, non-gravid females and gravid females. I predicted that the CORT response would be amplified at increased temperatures compared to the control temperature treatment ( $T_{\mathrm{b}}$ at capture) in all groups. At the same time, I examined whether gravid females are capable of mounting a significant CORT response during nesting when simultaneously exposed to capture-restraint and an acute temperature increase. A significant CORT response in gravid females would suggest that the ability to secrete CORT is not fundamentally impaired during the nesting life-history stage, but is seasonally modulated. 


\subsection{Materials and methods}

\subsubsection{Influence of natural variation in body temperature on CORT secretion}

\subsubsection{Study sites and animals}

Wild adult tuatara (Sphenodon punctatus) were captured and sampled from

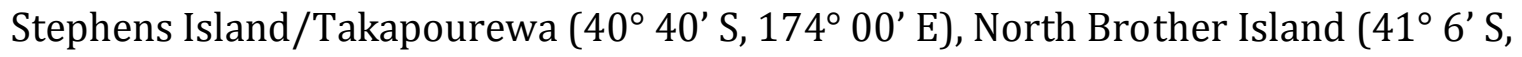
$174^{\circ} 25^{\prime} \mathrm{E}$ ), Lady Alice Island ( $35^{\circ} 53^{\prime} \mathrm{S}, 174^{\circ} 43^{\prime} \mathrm{E}$ ) and Taranga Island ('35 $55^{\circ} \mathrm{S}$, $174^{\circ} 43^{\prime} \mathrm{E}$ ) in New Zealand during the austral spring (November) and austral autumn (March) in the 2011/2012 and 2012/2013 seasons. Emerged individuals were captured by hand at night between 19:00 $\mathrm{h}$ and 03:00 $\mathrm{h}$ and were subsequently assigned to an appropriate group based on sex and reproductive status (male, non-gravid female, gravid female). Tuatara reach sexual maturity at approximately 13 years of age $(\sim$ SVL $\geq 170 \mathrm{~mm})$, and previous studies have distinguished males and females by examining secondary sex characteristics such as head and body size/shape and spine/crest morphology (Cree et al., 1991a; Dawbin, 1982). Gravid females (present in spring only) were selectively captured at or near a nesting rookery on Stephens Island/North Brother Island and reproductive status was inferred through abdominal palpation for shelled eggs (Mitchell et al., 2006; Refsnider et al., 2013). Locations of nesting rookeries on Lady Alice Island and Taranga Islands are not known; therefore, for the purpose of analyses, all females captured during the spring season on these two islands were classified as nongravid adults (although a proportion of sampled females could have been gravid). I intentionally include data from four island sites in order to expand the range of $T_{\mathrm{b}}$ observed, as samples collected within a site often showed limited variation in $T_{\mathrm{b}}$.

\subsubsection{Study design}

Male $(n=72)$, non-gravid female $(n=77)$ and gravid females $(n=30)$ were sampled over a two year study period to examine the influence of $T_{\mathrm{b}}$ on baseline CORT secretion and the CORT response across a wide range of environmental temperatures. Blood samples were collected within 10 min of capture to determine baseline CORT secretion (described in section 4.1.3) and tuatara were subsequently 
subject to $3 \mathrm{~h}$ of capture-restraint stress in cloth bags at ambient environmental temperatures. Upon completion of capture-restraint, all individuals were re-bled to determine the CORT response.

\subsubsection{Blood sampling protocol}

To determine baseline CORT concentrations, a blood sample (up to $1 \mathrm{ml}$ ) was collected within 10 min of capture from the base of the tail with a heparinized 23gauge needle and $1 \mathrm{ml}$ syringe. In previous studies of tuatara, no significant effect of bleeding effort (time from capture to blood sample collection) was observed on baseline CORT concentrations in blood samples collected within 20 min of capture (Chapter Two; Tyrell, 1993). After baseline samples were taken, individuals underwent $3 \mathrm{~h}$ capture-restraint whereupon a second blood sample (up to $1 \mathrm{ml}$ ) was taken. Depending on field conditions, blood samples were separated either by centrifuge ( $5 \mathrm{~min}$ at $2000 \mathrm{rpm}$ ) or under normal gravity for 6 to $8 \mathrm{~h}$ at $4^{\circ} \mathrm{C}$ (Reimers et al., 1983; Sheriff et al., 2011). Plasma was transferred into cryogenic vials with a micropipette, stored in a cryogenic dry shipper (Thermo Fisher Scientific ${ }^{\mathrm{TM}}$, Arctic Express $^{\mathrm{TM}}$ Dual 10, Waltham, Massachusetts, USA) or in a freezer at $-20^{\circ} \mathrm{C}$ until return to the laboratory, and then stored at $-80^{\circ} \mathrm{C}$ until assayed.

\subsubsection{Internal body temperature $\left(T_{b}\right)$ and body condition measurements}

Internal body temperature $\left(T_{\mathrm{b}}\right)$ was recorded with a cloacal thermocouple (Fluke ${ }^{\circledR}$ Multimeter, model: 179 , Everett, Washington, USA, reported accuracy $\pm 1^{\circ} \mathrm{C}$ ) prior to taking blood samples (both baseline and CORT response) for each individual. Morphometric measurements were taken after capture-restraint and CORT response blood sampling was completed. Individual animals were weighed with a spring scale (Pesola AG, Barr, Switzerland) to determine mass (g), and snout-vent length $(\mathrm{mm})$, tail length $(\mathrm{mm})$, and tail regeneration length $(\mathrm{mm})$ were measured with a ruler. I calculated tail-corrected mass (TCM) following Newman et al. (1994) to account for tail-loss and regeneration. Body condition scores for all individuals were obtained by generating standardized residuals from a regression of logTCM vs. 
log SVL for each group (males, non-gravid females, gravid females) (SchulteHostedde et al., 2005).

\subsubsection{Effect of acute increased body temperature on the CORT response}

\subsubsection{Study site and animals}

Wild adult tuatara were captured and sampled on Stephens Island $\left(40^{\circ} 40^{\prime} \mathrm{S}, 174^{\circ}\right.$ 00' E) in Cook Strait, New Zealand over 3 nights during the 2012 spring season (2124 October). Emerged individuals were caught by hand at night between 19:00 h and 03:00 $\mathrm{h}$ and were subsequently grouped by sex and reproductive status (male, non-gravid female, gravid female). Gravid females were selectively captured at a nesting rookery and reproductive status was inferred through abdominal palpation for shelled eggs.

\subsubsection{Study design}

Male $(n=14)$, non-gravid female $(n=14)$ and gravid female $(n=14)$ tuatara were subject to an acute increase in temperature to test whether the CORT response is amplified at higher temperatures. Blood samples were collected within $10 \mathrm{~min}$ of capture to determine baseline CORT secretion (described in section 3.3.1.3). Next, individuals were randomly assigned to an air temperature treatment of either 'control' (ambient), 'warm' $\left(20^{\circ} \mathrm{C}\right)$ or 'hot' $\left(25^{\circ} \mathrm{C}\right)$. Individuals were subject to $3 \mathrm{~h}$ of capture-restraint stress in perforated cardboard postal tubes $(10 \mathrm{~cm} \times 50 \mathrm{~cm})$ at assigned temperature treatments and upon completion, were re-bled to determine the effect of capture-restraint and temperature treatment on CORT secretion.

Internal $T_{\mathrm{b}}$ was taken immediately prior to each blood sample and body condition was determined for each individual (described in section 4.1.4) after the second blood sample was taken. Temperature treatments of $20^{\circ} \mathrm{C}$ and $25^{\circ} \mathrm{C}$ were chosen to approximate the average ('warm' treatment) and highest ('hot' treatment) $T_{\mathrm{b}}$, respectively, measured in the parallel observational study. These temperatures are physiologically appropriate (i.e. not considered lethal or expected to invoke signs of obvious 'heat-stress' such as panting) and fall within the range of $T_{\mathrm{b}}$ observed in 
(and selected by) individual tuatara in field and laboratory settings (Barwick, 1982; Besson and Cree, 2010; Corkery, 2012; Cree, 2014; Saint Girons, 1980; Werner and Whitaker, 1978). Treatment conditions were achieved by elevating air temperatures in a quiet confined space in the research house with convection heaters. Labelled postal tubes housing individuals were placed uniformly at an even height (established by a pilot study to determine optimal height for each temperature treatment), and air temperatures (within the grouping of postal tubes at each treatment height) for both treatments were monitored every 15-30 min with a thermocouple to ensure consistency of treatments. Individuals assigned to control treatments (ambient air temperature) were housed in postal tubes outside the research house.

\subsubsection{Determination of plasma CORT concentrations}

Plasma CORT concentrations for all samples were determined as described in Chapter Two. Briefly, I randomly selected plasma samples (29/plate) for assay that were thawed at room temperature and spun in a centrifuge at $5000 \mathrm{rpm}$ for $5 \mathrm{~min}$ at $4^{\circ} \mathrm{C}$ to remove any residual debris. I added $600 \mu \mathrm{l}$ of freshly distilled dichloromethane to extract $10 \mu \mathrm{l}$ aliquots of plasma samples. Samples were incubated $15 \mathrm{~min}$ at room temperature, snap-frozen in a $-70^{\circ} \mathrm{C}$ ethanol bath, decanted into glass tubes and then dried in a vacuum oven at $37^{\circ} \mathrm{C}$ for $2 \mathrm{~h}$. Samples were reconstituted with $120 \mu \mathrm{l}$ enzyme immunoassay (EIA) buffer (for a 12-fold dilution), vortexed and held at $4^{\circ} \mathrm{C}$ until assayed. I determined extraction efficiency by comparing mean recovery of extracted $(n=5)$ versus non-extracted $(n=5) 2000$ cpm tritiated CORT $\left(\mathrm{H}^{3}\right)$. Mean extraction efficiency of $\mathrm{H}^{3}$ was $106 \% \pm 2 \%$ s.d. $(n=16$ extractions) with an overall CV of $2 \%$.

Extracted plasma samples $(50 \mu \mathrm{l})$ were analysed in duplicate using commercial enzyme immunoassay kits (Cayman Chemical Co., Ann Arbor, MI, USA) containing 96-well plates coated with rabbit polyclonal anti-sheep IgG antibody. CORT-specific acetylcholinesterase (AChE) tracer and sheep CORT antiserum were added to sample wells, and the plate was placed on an orbital shaker for $2 \mathrm{~h}$. Plates were washed five times with wash buffer solution, developed for $1 \mathrm{~h}$ on an orbital shaker and subsequently read at $405 \mathrm{~nm}$. The concentration of CORT was calculated by 
comparing results to a standard curve. Samples that did not yield CORT concentrations within the $10-90 \%$ bound range were re-assayed after an appropriate dilution. The mean intra-assay CV was $10.9 \%$ and the mean inter-assay CV was $15.4 \%$.

\subsubsection{Statistical analyses}

Data analyses were carried out using R v3.2.0 statistical software (R Development Core 2013) and Prism 6 (Graphpad Software Inc.). Residual plots were checked for normal distributions and homoscedasticity, and if necessary, data were transformed to meet assumptions for parametric statistical tests. Linear mixed effects regression (LMER) models were fitted using the 'Ime4' package (Bates, 2013) in R to analyse 1) the relationship between $T_{\mathrm{b}}$ and CORT secretion across a range of ambient environmental temperatures, and 2) the effect of increased $T_{\mathrm{b}}$ on the CORT response in males, non-gravid females and gravid females subject to $3 \mathrm{~h}$ of capture-restraint stress in controlled temperature treatments. Log-transformed CORT was the response variable in all LMER models. Final model selection was determined by likelihood ratio tests comparing the addition of random and fixed effects to intercept-only baseline models.

In analysis 1 (influence of natural variation in $T_{\mathrm{b}}$ on CORT secretion), input variables included fixed main effects of time $(0 \mathrm{~h}, 3 \mathrm{~h}), T_{\mathrm{b}}$, body condition, and all interaction terms. Random effects of year and tuatara ID (nested within site) were included to account for annual variation, repeat sampling of individuals for baseline CORT and CORT response values, and random unmeasured variation between sites. Final models were fitted to data from each group for spring (males, non-gravid females, gravid females) and autumn (males, non-gravid females).

In analysis 2 (effect of increased $T_{\mathrm{b}}$ on the CORT response), input variables included fixed main effects of time $(0 \mathrm{~h}, 3 \mathrm{~h})$, body condition and temperature treatment (ambient, warm, hot), with all interaction terms included. A random effect of tuatara ID was included in both models to account for repeat sampling. Due to small sample size, I pooled data for males and non-gravid females for the experimental analyses, as sex was not a significant predictor in this study $(P>0.05)$; therefore final models 
were fitted to data from non-gravid adults (males, non-gravid females) and gravid adults (gravid females). Body condition did not have a significant influence on CORT secretion for any group $(P>0.05)$ and was not included in final models. I used linear models (LMs) and post-hoc contrast tests to compare $T_{\mathrm{b}}$ and body condition between groups within each controlled temperature treatment at $0 \mathrm{~h}$ and $3 \mathrm{~h}$ sample times, and I used a LMER model to compare change in $T_{\mathrm{b}}$ from $0 \mathrm{~h}$ to $3 \mathrm{~h}$ in each temperature treatment (with tuatara ID included as a random effect to account for repeat sampling). The 'ImerTest' package (Kuznetsova, 2013) was used to compute $P$-values for final models and significance was assumed at $P<0.05$.

\subsection{Results}

\subsubsection{Influence of natural variation in body temperature on CORT secretion}

Internal $\mathrm{T}_{\mathrm{b}}$ ranged from $8^{\circ} \mathrm{C}$ to $20^{\circ} \mathrm{C}$ in spring and $12^{\circ} \mathrm{C}$ to $23^{\circ} \mathrm{C}$ in autumn (Fig. 3.1). Body temperature at capture $(0 \mathrm{~h})$ and at $3 \mathrm{~h}$ after capture was not significantly different among groups (males, non-gravid females, gravid females) in either season (LM, $P>0.05$ ). However, $T_{\mathrm{b}}$ was significantly increased at $3 \mathrm{~h}$ after capture (LMER, $P<0.001$ ), which is most likely due to sheltered conditions (in capture bags) (Chapter Two; Tyrrell and Cree, 1998).

As expected, I observed a significant CORT response to capture restraint in males and non-gravid females in both seasons (Fig. 3.1, Table 3.1), and observed a dampened CORT response in gravid females in spring (Fig. 3.1, Table 3.1a). I found that $T_{\mathrm{b}}$ was not correlated with baseline CORT in males and non-gravid females during spring or autumn (Table 3.1); however, $T_{\mathrm{b}}$ is positively correlated with CORT secretion in gravid females in spring (Table 3.1a, Fig. 3.1a). Furthermore, a higher CORT response was observed at higher $T_{\mathrm{b}}$ in all groups in each season (including gravid females - present in spring only) (Fig. 3.1b, d, Table 3.1).

Body condition scores were not significantly different between seasons (spring, autumn) or among groups (male, non-gravid female, gravid female) (LM, $F_{4}$, $355=0.309, P=0.872$ ). Body condition was not correlated with baseline CORT for any 
group/season (LMER, $P>0.05$ ). However, in spring only, a significant positive correlation was observed between body condition and the CORT response in nongravid females (LMER, $P<0.001$ ) and in gravid females (LMER, $P=0.035$ ), with a weak trend observed in males (LMER, $P=0.09$ ). No significant interactions between $T_{\mathrm{b}}$ and body condition were observed for any group/season (LMER, $P>0.05$, Table 3.1).

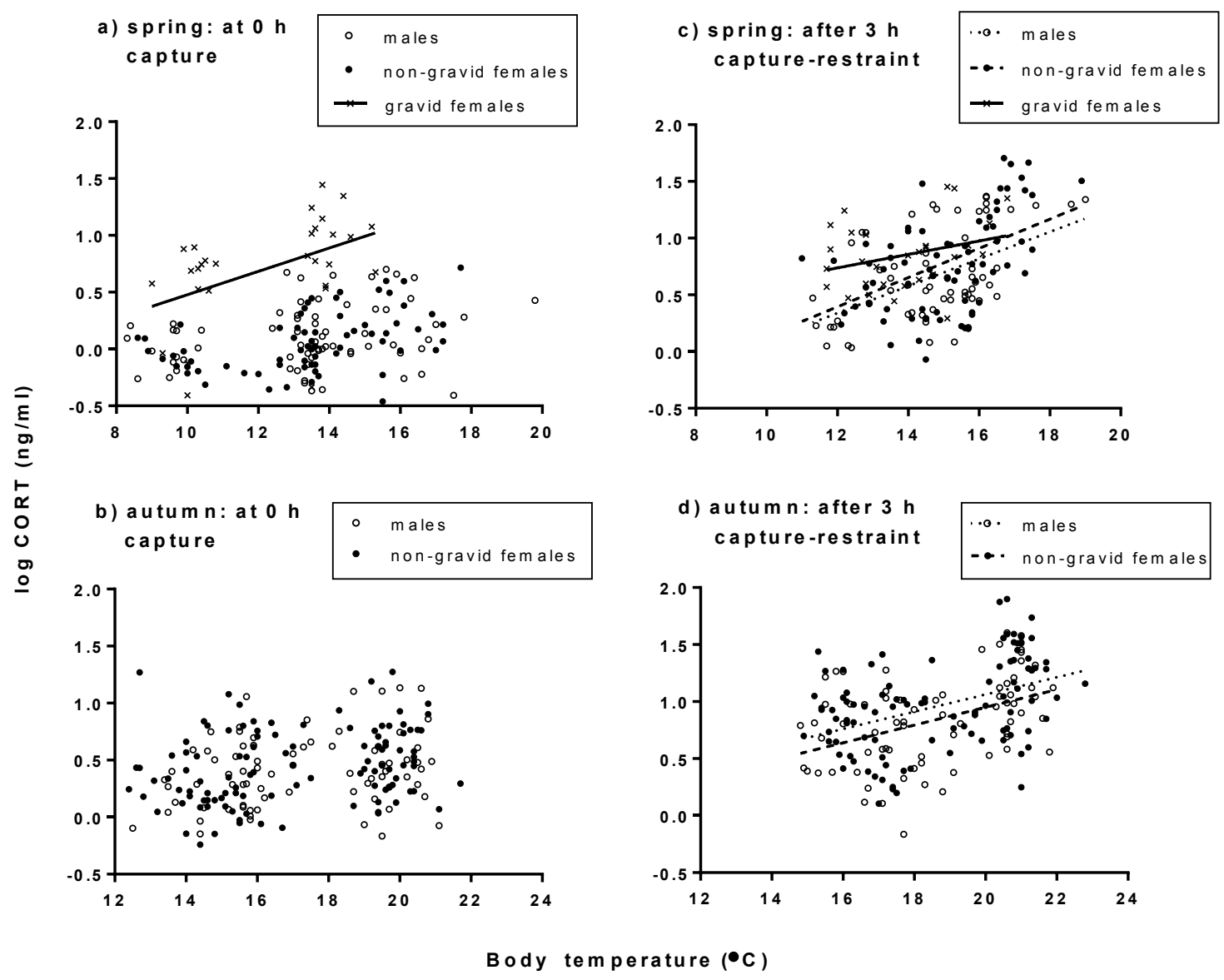

Figure 3.1: Relationships between internal body temperature $\left({ }^{\circ} \mathrm{C}\right)$ and $\log$ CORT concentration ( $\mathrm{ng} / \mathrm{ml}$ ) in tuatara (Sphenodon punctatus). Data are shown for male (open circles, dotted lines), non-gravid female (black circles, dashed lines) and gravid female (crosses, solid lines) tuatara at $\mathrm{O} \mathrm{h}$ after capture in a) spring and b) autumn and after $3 \mathrm{~h}$ capture-restraint in c) spring and d) autumn. Data points represent each individual sampled and regression lines show positive relationships. Note: gravid females are only present during spring. 
Table 3.1: Results from LMER models explaining variation in baseline CORT and the CORT response in tuatara (S. punctatus) accounting for fixed effects of time ( $0 \mathrm{~h}$ capture and $3 \mathrm{~h}$ capture-restraint), internal body temperature, body condition and the interaction of these effects (if applicable). Models were fitted to data for males, non-gravid females, and gravid females in spring (a), and for males and non-gravid females in autumn (b). ${ }^{1}$ Gravid adults are present in spring season only.

\section{Fixed effect predictor}

a) Spring

\section{males}

$0 \mathrm{~h}$ (Intercept)

$3 \mathrm{~h}$

$T_{\mathrm{b}}$

body condition

$3 \mathrm{~h}$ x body condition

$3 \mathrm{~h} \times T_{\mathrm{b}}$

$T_{\mathrm{b}} \mathrm{X}$ body condition

$3 \mathrm{~h} \times T_{\mathrm{b}} \mathrm{x}$ body condition

\section{Non-gravid females}

$0 \mathrm{~h}$ (Intercept)

$3 \mathrm{~h}$

$T_{\mathrm{b}}$

body condition

$3 \mathrm{~h} \mathrm{x}$ body condition

$3 \mathrm{~h} \times T_{\mathrm{b}}$

$T_{\mathrm{b}} \mathrm{x}$ body condition

$3 \mathrm{~h} \times T_{\mathrm{b}} \mathrm{x}$ body condition

\section{gravid females $^{1}$}

$0 \mathrm{~h}$ (Intercept)

$3 \mathrm{~h}$

$T_{\mathrm{b}}$

body condition estimate

$\begin{array}{rrrc}0.198 & 0.162 & 1.215 & 0.378 \\ 0.571 & 0.063 & 9.027 & <0.001^{*} \\ -0.029 & 0.199 & -1.428 & 0.156 \\ -0.028 & 0.052 & -0.527 & 0.599 \\ 0.112 & 0.066 & 1.691 & 0.093 \\ 0.116 & 0.028 & 4.049 & <0.001^{*} \\ 0.021 & 0.019 & 1.115 & 0.267 \\ -0.004 & 0.030 & -0.134 & 0.893\end{array}$

0.349

0.631

0.273

0.049

$-0.004$

0.017

$-0.046$

0.040

0.210

0.059

0.084

0.021

$-0.013$

0.022

$-0.023$

0.035

1.279

12.867

0.367

$-0.291$

$<0.001^{*}$

$-1.146$

0.771

0.254

3.552

$<0.001^{*}$

$3.937<0.001^{*}$

$-0.593$

0.554

$-0.680$

0.498

$\begin{array}{rrrc}0.780 & 0.064 & 12.074 & <0.001 \\ -0.027 & 0.070 & -0.383 & 0.704 \\ 0.109 & 0.026 & 4.143 & <0.001^{*} \\ 0.121 & 0.054 & 2.233 & 0.035^{*}\end{array}$

b) Autumn

\section{Males}

$\begin{array}{lcccc}0 \mathrm{~h} \text { (Intercept) } & 0.430 & 0.144 & 2.967 & 0.171 \\ 3 \mathrm{~h} & 0.349 & 0.055 & 6.246 & <0.001^{*} \\ T_{\mathrm{b}} & 0.018 & 0.017 & 1.056 & 0.334 \\ 3 \mathrm{~h} \times T_{\mathrm{b}} & 0.055 & 0.021 & 2.561 & 0.012^{*}\end{array}$

\section{Non-gravid females}

\begin{tabular}{lccrc}
$0 \mathrm{~h}$ (Intercept) & 0.396 & 0.168 & 2.354 & 0.086 \\
$3 \mathrm{~h}$ & 0.545 & 0.050 & 10.791 & $<0.001^{*}$ \\
$T_{\mathrm{b}}$ & -0.017 & 0.019 & -0.855 & 0.378 \\
$3 \mathrm{~h} \times T_{\mathrm{b}}$ & 0.031 & 0.018 & 1.740 & 0.084 \\
\hline
\end{tabular}




\subsubsection{Effect of acute increased body temperature on the CORT response}

Body temperature at capture $(0 \mathrm{~h})$ was comparable between groups assigned to all temperature treatments ( $\mathrm{LM}, P>0.05)$. As expected, significantly higher $T_{\mathrm{b}}$ was reached at $3 \mathrm{~h}$ in the 'warm' and 'hot' temperature treatments, compared to the ambient 'control' treatment (LMER, $P<0.05$, Fig. 3.2a).

The increase in $T_{\mathrm{b}}$ was not significantly different among groups (males, non-gravid females, gravid females) within each temperature treatment at $3 \mathrm{~h}(\mathrm{LM}, P>0.05)$. Mean $T_{\mathrm{b}}$ after $3 \mathrm{~h}$ reached $12.6 \pm 0.7^{\circ} \mathrm{C}$ in the 'control' treatment, $17.3 \pm 0.4^{\circ} \mathrm{C}$ in the 'warm' treatment and $21.4 \pm 0.4^{\circ} \mathrm{C}$ (which is $T_{\text {sel }}$ for tuatara) in the 'hot' temperature treatment (Fig. 3.2a). Body condition was comparable among groups ( $\mathrm{LM}, P>0.05$ ), and was not a significant predictor of CORT secretion in this experiment (LMER, $P>0.05)$.

Males and non-gravid females (pooled data) exhibited a significant CORT response in all temperature treatments (Fig. 3.2b, Table 3.2a). The magnitude of the CORT response was not significantly different between the 'control' and 'warm' treatments (LMER, $P>0.05$ ); however, a positive trend in the 'hot' temperature treatment was observed and suggests an amplified CORT response in this treatment, compared to 'control' and 'warm' treatments (LMER, $P=0.09$ ) (Fig. 3.2b, Table 3.2a). Gravid females maintained a dampened CORT response in the 'control' and 'warm' temperature treatments (LMER, $P>0.05$ ), but exhibited a significant CORT response in the 'hot' temperature treatment (LMER, $P=0.04$ ) (Fig. 3.2c, Table 3.2b). 

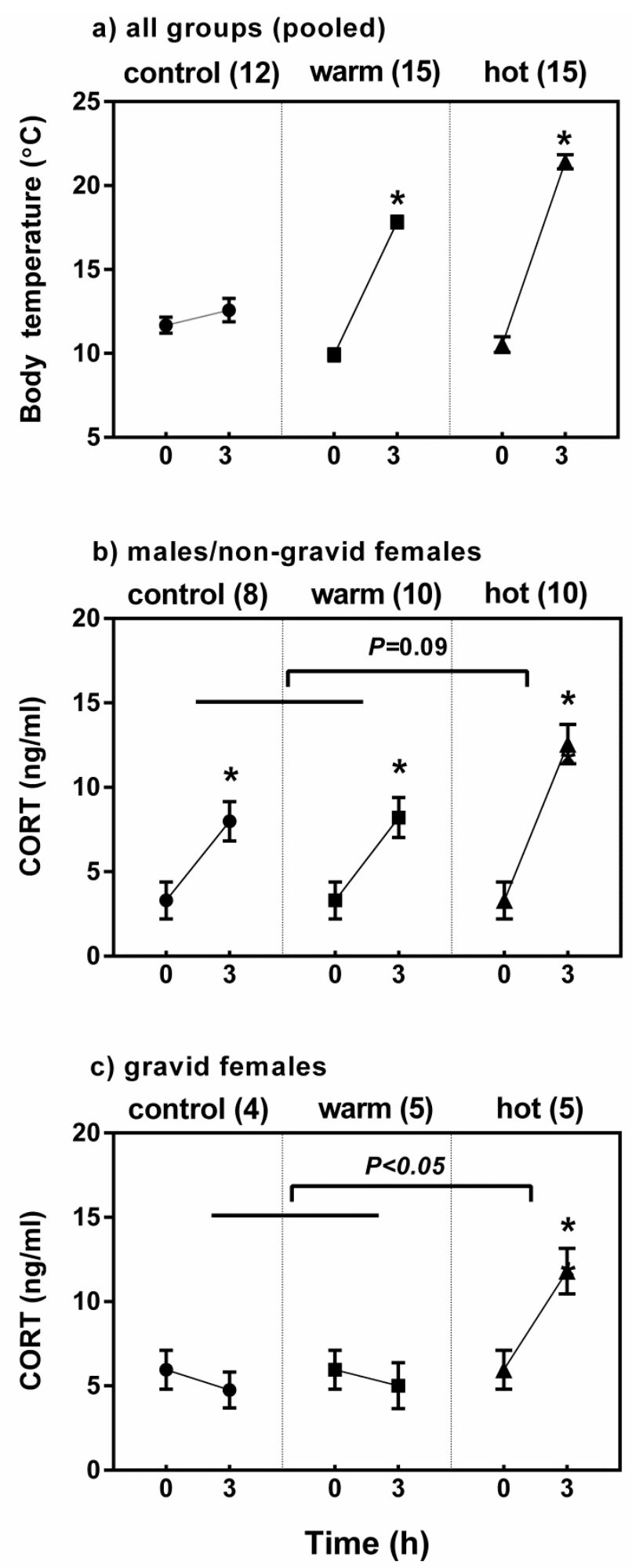

Figure 3.2: Effect of acute increased body temperature on the CORT response in tuatara (Sphenodon punctatus). Data show change from $0 \mathrm{~h}$ to $3 \mathrm{~h}$ in a) internal body temperature, CORT secretion in b) male and non-gravid female and c) gravid female tuatara subject to capturerestraint and temperature treatments of 'control' (mean $T_{\mathrm{b}}=12.6^{\circ} \mathrm{C}$ ), 'warm' (mean $T_{\mathrm{b}}=17.3^{\circ} \mathrm{C}$ ) and 'hot' (mean $T_{\mathrm{b}}=21.4^{\circ} \mathrm{C}$ ). Data points represent mean \pm s.e. Asterisks indicate a significant change observed after $3 \mathrm{~h}$ compared to baseline $(0 \mathrm{~h})$, and bars above treatments show the difference in magnitude of the CORT response. Numbers in brackets indicate sample size. 
Table 3.2: Results from LMER models explaining variation in measures of baseline CORT and the CORT response in tuatara (S.punctatus) accounting for fixed effects of time, temperature treatment (control, warm, hot) and the interaction of these effects for a) male/non-gravid female tuatara and b) gravid female tuatara.

\section{Fixed effect predictor}

a) males/non-gravid females

$0 \mathrm{~h}$ control (Intercept)

0 h warm

$0 \mathrm{~h}$ hot

$3 \mathrm{~h} \times$ control

$3 \mathrm{~h} \times$ warm

$3 \mathrm{~h} \times$ hot
0.560

$-0.084$

$-0.027$

0.343

0.096

0.223
0.673

0.101

0.105

0.097

0.126

0.132

\section{$T$ value $P$ value}

b) gravid females

$\begin{array}{lcccc}\text { 0 h control (Intercept) } & 0.823 & 0.121 & 6.818 & <0.001 \\ \text { 0 h warm } & -0.217 & 0.171 & -1.275 & 0.218 \\ \text { 0 h hot } & 0.034 & 0.155 & 0.219 & 0.828 \\ 3 \mathrm{~h} \text { x control } & -0.145 & 0.124 & -1.166 & 0.268 \\ 3 \mathrm{~h} \times \text { warm } & 0.096 & 0.126 & 0.076 & 0.454 \\ 3 \mathrm{~h} \times \text { hot } & 0.360 & 0.161 & 2.243 & 0.046^{*}\end{array}$

\subsection{Discussion}

I examined the relationship between natural variation in ambient temperature and baseline CORT and the CORT response in tuatara, and experimentally tested whether acute increased body temperature affects the magnitude of the CORT response. Here, I confirm for a cold-adapted reptile a significant correlation between body temperature and CORT secretion. My results reveal three interesting patterns: (i) a significant positive correlation between $T_{\mathrm{b}}$ and baseline CORT secretion in gravid females only, (ii) a significant positive correlation between $T_{\mathrm{b}}$ and the CORT response in males and females (gravid and non-gravid), and (iii) an acute increase in $T_{\mathrm{b}}$ approaching a threshold at/near ambient temperatures of $25^{\circ} \mathrm{C}$ elicits a significant CORT response in gravid females (which at lower temperatures fail to show a response), and may influence the magnitude of the CORT response in 
males and non-gravid females (though the latter is a marginal trend). These findings are important as many behavioural and physiological processes in ectotherms are sensitive to changes in ambient environmental temperature (Bonnet et al., 2013; Cartland and Grimmond, 1994; Finkler, 2006; Gaby et al., 2011; Lawrence, 1997; Lourdais et al., 2008), and should therefore be considered in physiological studies utilising ectotherms as model organisms.

\subsubsection{Body temperature is correlated with baseline CORT in gravid females only}

I observed a positive correlation between $T_{\mathrm{b}}$ and baseline CORT in gravid females only, and found no significant correlation between $T_{\mathrm{b}}$ and baseline CORT in males or non-gravid females. These results agree with findings from a previous study investigating patterns of CORT in male, non-gravid female and gravid female tuatara (Chapter Two). Other studies have reported inconsistent effects of $T_{\mathrm{b}}$ on baseline CORT in reptiles, with reports of a positive relationship, negative relationship or no relationship at all (Baker et al., 2013; Romero and Wikelski, 2006). Tyrrell and Cree (1998) found a positive correlation between $T_{\mathrm{b}}$ and baseline CORT in male but not female tuatara, during summer and winter months, which the authors suggested could be a product of increased $T_{\mathrm{b}}$ promoting specific behavioural activity that coincides with increased baseline CORT. This hypothesis could potentially explain the elevated baseline CORT observed in gravid females in the current study, i.e. it is possible that higher $T_{\mathrm{b}}$ in gravid females may facilitate (or be connected with) migration to nesting grounds, nest digging and/or oviposition activities in the spring nesting season. Cree and Tyrrell (2001) associated nesting behaviour (digging/nest guarding) with elevated baseline CORT in gravid female tuatara; however $T_{\mathrm{b}}$ was not measured/reported in that study. Therefore, further research is required to better understand the relationship and connection between $T_{\mathrm{b}}$, behavioural activities, and baseline CORT.

\subsubsection{The CORT response is higher at warmer ambient temperatures}

The magnitude of the CORT response was significantly higher in tuatara sampled at warmer ambient temperatures, with effectively higher $T_{\mathrm{b}}$. Numerous studies have 
reported increased rates for physiological processes at warmer temperatures in reptiles, including hormone secretion (Callard et al., 1975; Narayan and Hero, 2014), blood circulation (Dunlap, 2006; Maclean et al., 1975), gestation (Michel et al., 2013), and metabolic processes such as growth, digestion and locomotion (Aidam et al., 2013; Bonnet et al., 2013; Cartland and Grimmond, 1994; Gillooly et al., 2001; Narayan et al., 2012; Polo-Cavia et al., 2012; Preest and Cree, 2008; Tamplin et al., 2013).

In addition, I found that body condition is positively correlated with the CORT response in the spring season, but not in the autumn season. In tuatara, it is possible that a stronger CORT response may contribute to better body condition in the spring. Food resources are reduced during the winter period, with a seasonal shift of increased prey availability occurring in late spring/summer (Walls, 1981); therefore, the presence of a stronger CORT response may reflect increased foraging activity in certain individuals leading to better body condition. Many studies have found a positive correlation between CORT secretion and feeding behaviour (reviewed in Landys et al., 2006), and experimentally elevated CORT levels have been shown to stimulate foraging activities (Breuner and Hahn, 2003; Crossin et al., 2012; Lancaster et al., 2008). Therefore, it may be beneficial to have a stronger CORT response during specific seasons, environmental conditions and/or lifehistory events. Alternatively, my results may indicate that individuals in better body condition are able to mount a stronger CORT response compared to individuals with reduced body condition. This prediction could be examined by looking at the CORT response across a range of body conditions, seasons and environmental scenarios (see Chapter Four). The role of CORT in regulating foraging activity could be examined experimentally in a controlled setting by applying exogenous CORT and monitoring feeding behaviour. Results from such studies may clarify the relationship between body condition and the magnitude of the CORT response.

\subsubsection{Acute increased body temperature elicits a CORT response in gravid females}

In my experimental study, I confirmed that an acute increase in $T_{\mathrm{b}}$ can elicit a CORT response in gravid females. I did not observe a significant CORT response in gravid 
females in both 'control' and 'warm' temperature treatments; yet a significant CORT response was exhibited by gravid females in the 'hot' temperature treatment. My experiment demonstrates, as previously suggested (Chapter Two), that gravid females are not operating at maximal CORT secretion at capture (despite their high baseline CORT levels) and are indeed able to mount a significant CORT response during the nesting life-history stage. In addition, I also observed a weak trend between an acute increase in $T_{\mathrm{b}}$ and the CORT response in males and non-gravid females in the 'hot' temperature treatment, though the trend status is marginal. I recognise that my sample sizes are small (particularly for gravid females); therefore it would be useful to conduct a larger-scale experiment in which $T_{\mathrm{b}}$ extends slightly above $T_{\text {sel }}$ to confirm and extend this result.

Nevertheless, the significant findings observed in gravid females in my experimental study, paired with the weak trend observed in males and non-gravid females, provides compelling evidence that exposure to acute temperature increases (up to and most likely above $T_{\text {sel }}$ ) can increase the magnitude of the CORT response in a cold-adapted reptile. This may be explained by an increase in the rate of hormone secretion at higher temperatures (as discussed in section 3.5.2), or possibly an effect of exposure to compound stressors (capture-restraint plus $T_{\mathrm{b}}$ increase/inability to thermoregulate). In a natural setting, ectotherms have the capacity to buffer changes in ambient air temperatures (and thus actively thermoregulate $T_{\mathrm{b}}$ ) through behavioural actions such as seeking shade, retreating to burrows, or basking (Besson and Cree, 2010; Kearney et al., 2009; Seebacher et al., 1999); therefore, exposure to increased temperatures (without the option to regulate $T_{\mathrm{b}}$ ) may explain my findings.

In a similar study to mine, the Children's python (Antaresia childreni) exhibited an higher CORT response when subject to a cold temperature treatment of $17^{\circ} \mathrm{C}$ (which is well below the pythons' $T_{\text {sel }}$ of $\sim 29^{\circ} \mathrm{C}$ ). The authors suggest a response threshold exists to allow individuals to cope with suboptimal temperatures when unable to thermoregulate. It would be interesting to see if the same response was observed at higher $T_{\mathrm{b}}$ (above $T_{\text {sel }}$ ) in this species. Likewise, the magnitude of the CORT response to suboptimal temperatures could be tested in tuatara by decreasing $T_{\mathrm{b}}$. However, as 
tuatara are a cold-adapted reptile and have been observed at ambient temperatures as low as $5-6^{\circ} \mathrm{C}$, with a critical thermal minimum temperature of $0.7^{\circ} \mathrm{C}$ (Besson and Cree, 2011), it would be likely that a 'cool' temperature for tuatara could possibly approach $0^{\circ} \mathrm{C}$ and there could be a risk of tissue/cell freezing (Gillooly et al., 2001). In a recent study on the Qinghai toad-headed lizard (Phrynocephalus vlangalii) (a cold-adapted alpine reptile with ambient daytime $T_{\mathrm{b}}$ ranging from $0-20^{\circ} \mathrm{C}$ ), CORT secretion was examined after exposure to acute cold-temperature treatments (at $0.1-0.5^{\circ} \mathrm{C}$ ) of varying durations. No significant response to temperature treatments was observed; however, the lizards used in this study were wild-caught, held in captivity for two weeks before the experiment and neither baseline CORT secretion values, the effect of captivity nor inter-individual fights were considered, which may have contributed to the observed results (Li et al., 2011). Similarly, the influence of both 'cold' and 'warm' temperatures on the CORT response in water snakes (Nerodia sipedon) was investigated (in which acute change from $T_{\mathrm{b}}$ at capture was $\pm 10^{\circ} \mathrm{C}$ ), and no effect of acute temperature on the magnitude of the CORT response was observed (Sykes and Klukowski, 2009). Water snakes are known to regularly experience acute temperature change (i.e. moving from basking to swimming) and are able to function over a wide range of $T_{\mathrm{b}}\left(\sim 7-30^{\circ} \mathrm{C}\right)$, which may explain the lack of response to temperature in this species. Clearly, more research investigating the interplay between temperature, thermoregulatory capacity, and CORT secretion in ectotherms is needed.

Increased CORT secretion supports immediate survival during situations where challenges are numerous or perceived as more intense/stressful (Bonnet et al., 2013; Graham et al., 2012). I recommend the examination of nesting behaviour (digging, oviposition, guarding etc.) of gravid female tuatara to test the prediction that they will abandon (or possibly will not initiate) nesting activities at a specific temperature threshold (at or above $T_{\text {sel }}$ ) at which a significant CORT response is produced. Studies in green turtles have reported that gravid females exposed to heat-stress or severe shark attacks maintain a dampened CORT response and continue nesting (Jessop et al., 2004a; Jessop, 2001). 


\subsection{Conclusions}

I confirm a significant correlation between $T_{\mathrm{b}}$ and CORT secretion in a cold-adapted ectotherm. The magnitude of the CORT response is positively correlated with ambient temperatures in male and female (gravid and non-gravid) tuatara, whereas baseline CORT is positively correlated with ambient temperatures in gravid females only. An acute increase in $T_{\mathrm{b}}$ to $T_{\text {sel }}\left(\sim 21^{\circ} \mathrm{C} T_{\mathrm{b}}\right)$ elicits a significant CORT response in gravid female tuatara, and may increase the CORT response in males and non-gravid females (although this is a weak trend). Possible explanations for this observation include a CORT response threshold at $T_{\text {sel, }}$ an increase in the rate of hormone secretion caused by higher temperatures, or an effect of compound stressors (capture-restraint plus $T_{\mathrm{b}}$ increase /inability to thermoregulate). These findings are important as many behavioural and physiological processes in ectotherms are sensitive to changes in ambient temperature and should therefore be considered in physiological studies utilising ectotherms as model organisms, especially where variation in ambient temperatures is experienced. Furthermore, these results will inform conservation efforts involving capture-restraint (which constrains normal thermoregulatory behaviour), such as in population monitoring, research programmes, translocation and captive management programmes. 


\section{The corticosterone stress response varies among island populations of tuatara (Sphenodon punctatus) and is associated with linear ecological attributes ${ }^{1}$}

\subsection{Abstract}

The most widely used indicators of physiological stress in free-living vertebrates are glucocorticoid (GC) hormones. Measurement of GC concentrations in body fluids or products to assess population status and/or to monitor conservation efforts is central to stress physiology research. However, in order to fully understand the stress physiology of a species, and to make comparisons among populations, it is essential to consider seasonal patterns in GC secretion and incorporate ecological variation. I compared baseline corticosterone (CORT), the primary GC in reptiles, and the $3 \mathrm{~h}$ CORT response to capture-restraint among four populations of a rare rhynchocephalian reptile, the tuatara (Sphenodon punctatus) during the nonbreeding and breeding seasons to determine inter-population variation in CORT secretion. Then, I used principal components analysis (PCA) to simplify five ecological attributes into two component axes that explained $96 \%$ of the ecological variance among populations, and used principal component regression to test linear ecological predictors (PC1, PC2) of CORT secretion. Finally, I determined plasma testosterone (T) in a subset of two populations as a proxy for reproductive activity

\footnotetext{
${ }^{1}$ This chapter is based on the following manuscript submitted to Animal Conservation (in review) with minor modifications: Anderson L, Nelson N, Towns D, Cree A. The corticosterone stress response varies among island populations of tuatara (Sphenodon punctatus) and is predicted by linear ecological attributes.
} 
and examined associations between T and CORT secretion. I found that baseline CORT secretion was similar among populations during the non-breeding and breeding seasons; however, the CORT response to capture-restraint varied significantly among populations. In general, baseline CORT increased, and the CORT response decreased, from the non-breeding season to the breeding season. I found significant correlations between the CORT response and habitat factors of latitude, tuatara density and seabird abundance (PC1 axis) and demogenetic factors of sex ratio and genetic diversity (PC2 axis); however, no correlations were observed between baseline CORT along both axes. Testosterone secretion was not associated with CORT secretion. Nonetheless, inter-population variation in T was observed in males (but not females) and was positively linked with increased baseline CORT from the non-breeding season to the breeding season, suggesting that male reproductive activity may drive the seasonal increase in baseline CORT.

\subsection{Introduction}

Glucocorticoid hormones (corticosterone (CORT) or cortisol) are the most widely used indicators of physiological stress in free-living vertebrates (Wikelski and Cooke, 2006). The use of CORT in body fluids (plasma) or products (urine, faeces) as a rapid tool to assess population health and/or to monitor conservation efforts is instrumental in stress physiology research (Busch and Hayward, 2009; Dantzer et al., 2014; Wikelski and Cooke, 2006). When individuals experience unpredictable challenges (i.e. stressors) that affect internal balance or homeostasis, a complex physiological response cascades through the hypothalamo-pituitary-adrenal (HPA) axis to stimulate CORT secretion (as either a gradual increase in baseline CORT or as an immediate CORT stress-response) to help individuals cope with stressors and to promote overall fitness and survival (Wingfield and Kitaysky, 2002; Wingfield et al., 1998). Even so, patterns of CORT secretion are not universally consistent; variation is observed among species, and within and among populations (Cockrem, 2013; Creel et al., 2013; Love et al., 2013). 
Variation in CORT secretion can be linked to intrinsic factors (sex, reproductive status, age) and to environmental, social and/or physiological stressors such as habitat modification (either natural or anthropogenic causes), as well as seasonal changes in food availability, temperature and/or weather, and in reaction to increased energy demands required during reproduction, territorial disputes and migration (Baker et al., 2013; Berner et al., 2013; Hamann et al., 2007; Holding et al., 2014b; Owen et al., 2014; Rubenstein and Wikelski, 2005; Taylor et al., 2014; Woodley et al., 2003).

It is generally agreed that baseline CORT meets energetic demands associated with predictable life-history stages, whereas the CORT response is a reaction to unpredictable and/or challenging events (Tokarz and Summers, 2011; Wingfield et al., 1998). Modulation of CORT secretion helps individuals cope with their specific environment to effectively increase fitness (Moore and Jessop, 2003; Romero, 2002). Baseline CORT is commonly elevated during the breeding season to support energetic requirements associated with reproductive activities, and similarly, individuals experiencing reduced breeding opportunities or living in harsh environments have been found to dampen their CORT response during the breeding season, which may serve to increase chances of reproductive success (Ebensperger et al., 2013; Holding et al., 2014b; Jessop et al., 2000; Moore and Jessop, 2003; Romero, 2006; Wingfield et al., 1998; Wingfield and Sapolsky, 2003).

Examining patterns of CORT secretion within a population can be a useful tool to assess and monitor overall health, and may be a way to identify unpredicted challenges and gauge an individual's ability to respond to stressors (Bonier et al., 2009; Breuner et al., 2008). That said, gaining a good understanding of factors that influence CORT secretion, while considering environmental context, is important. Many studies have examined factors influencing CORT secretion (both baseline CORT and the CORT response); however, studies that incorporate intrinsic, seasonal, and ecological/anthropogenic attributes to assess sources of variation in CORT secretion among wild populations are limited (Boonstra, 2013; Dantzer et al., 2014). 
Here, I compare patterns in the secretion of CORT, the main GC in reptiles, among four wild populations of tuatara (Sphenodon punctatus) during the non-breeding and breeding seasons, and examine potential attributes that may explain variation in CORT secretion. Natural populations of tuatara are currently restricted to isolated offshore islands (Jones and Cree, 2012) with considerable variation in ecological attributes such as island size, sex ratio, body condition, climate, conspecific density, seabird abundance, food availability, human activity, history of introduced Pacific rats (Rattus exulans), genetic diversity and demography (Cree, 2014; Gaze, 2001). Tuatara are an ideal species to examine relationships between CORT secretion and influential factors (intrinsic, seasonal, ecological/anthropogenic) due to the variety of environmental conditions observed among island populations and to their distinctive breeding cycle.

Tuatara have seasonally distinct reproductive activity with breeding (mating and ovulation) occurring in autumn (February - March) and nesting in spring (October December), though not all females ovulate and nest each year (Cree, 2014). Male reproductive activity peaks in late summer/early autumn (January-March) (Saint Girons and Newman, 1987) and includes prenuptial displays, courtship, mating, and territorial defence (Gans et al., 1984; Moore et al., 2009b). Male reproductive success is highly skewed, with only $25-30 \%$ of individuals successful in securing a mate (Moore et al., 2009a). Spermatogenesis and plasma testosterone concentrations in males follow an annual cycle, being low during the winter, rising in the spring, and peaking in midsummer to early autumn during the mating period (Cree, 2014). Female tuatara exhibit an extended reproductive cycle, carrying eggs in the oviduct for 6-8 months and producing one clutch of eggs every 2-9 years, depending on the population (Cree et al., 1992; Moore et al., 2009a; Refsnider et al., 2010). Female tuatara show highest levels of testosterone and estradiol when in pre-ovulatory condition during the breeding season, however; only 10 - 50\% of females in a given population are ovulating (hence receptive to a mate) during the breeding season (Cree, 2014; Mitchell et al., 2010; Moore et al., 2009a; Tyrrell et al., 2000). 
Baseline CORT in tuatara is generally low (Cree and Tyrrell, 2001; Tyrrell and Cree, 1998) and a significant CORT response is observed to capture-restraint (Chapter Two; Tyrrell and Cree, 1998). Studies examining variation in CORT secretion among tuatara populations are limited, with only one previous study comparing CORT secretion between two northern populations (a rat-free population vs a ratinhabited population), in which presence of rats was associated with an elevation in the $3 \mathrm{~h}$ and $18 \mathrm{~h}$ CORT response but did not influence baseline CORT (Tyrrell et al., 2000).

Seasonal variation in baseline CORT has been observed in the population on Stephens Island, where baseline CORT was highest in November for females and February/May for males (Tyrrell and Cree, 1998); however, seasonal patterns of baseline CORT secretion (in other island populations) and seasonal patterns of the CORT response have not been investigated. I found a positive correlation between body temperature ( $\left.T_{\mathrm{b}}\right)$ and the CORT response in tuatara (Chapter Three), and that body condition is not correlated with baseline CORT or the CORT response (Chapter Two; Chapter Three). Here, I make further use of samples collected in Chapter Three (along with new samples) to examine inter-island variation of CORT secretion in two seasons, while effectively controlling for (and examining) the influence of temperature and body condition.

In this study, I examined variation in CORT secretion (baseline CORT and the CORT response to capture-restraint) among four populations during the non-breeding and breeding seasons. I predicted that (i) baseline CORT would vary among populations during each season due to contrasting environmental attributes; (ii) baseline CORT would be higher during the breeding season (compared to the non-breeding season) in all populations, as tuatara exhibit energetically demanding reproductive behaviours/activity during the breeding season (Moore et al., 2009b; Saint Girons and Newman, 1987); and, (iii) the CORT response would be higher in northern populations (compared to Cook Strait populations), in both seasons, due to a milder climate (e.g. warmer $T_{\mathrm{b}}$ ) and past/recent rat presence. I tested relevant ecological predictors of CORT secretion (through principal component analysis) to identify significant factors that may contribute to variation among populations. Secondly, I 
examined the association between reproductive activity (as indicated by testosterone (T) concentrations) and modulation of CORT secretion from the nonbreeding season to the breeding season in a subset of two populations with contrasting seasonal modulation of baseline CORT.

\subsection{Material and methods}

\subsubsection{Study sites and animals}

Wild adult tuatara (Sphenodon punctatus) were captured and sampled from four island populations in New Zealand during the non-breeding (austral spring) and breeding (austral autumn) seasons. Samples were collected from Stephens Island/Takapourewa ( $40^{\circ} 40^{\prime} \mathrm{S}, 174^{\circ} 00^{\prime} \mathrm{E}$ ), North Brother Island ( $41^{\circ} 6^{\prime} \mathrm{S}, 174^{\circ} 25^{\prime}$ E), Lady Alice Island ( $35^{\circ} 53^{\prime} \mathrm{S}, 174^{\circ} 43^{\prime} \mathrm{E}$ ) and Taranga Island (' $35^{\circ} 58^{\prime} \mathrm{S}, 174^{\circ} 43^{\prime}$ E) during the non-breeding season (November 2011) and breeding season (March $2011^{*}$ and March 2012, ${ }^{*}$ Lady Alice and Taranga Islands only). Both Lady Alice Island and Taranga Island CORT concentrations were not significantly different between the 2011 and 2012 breeding seasons (LMER, $P>0.05$ ), therefore I pooled $2011 / 2012$ breeding season samples for each site to increase sample size for analyses.

Samples were obtained from adult males and adult non-gravid females during each season. Tuatara reach sexual maturity at approximately 13 years of age ( $\sim$ SVL $\geq$ $170 \mathrm{~mm}$ ) (Castanet et al., 1988), at which point sex can be identified by examining head shape, spine shape, and body size/shape (Cree, 2014; Cree et al., 1991a; Dawbin, 1982). Gravid females with eggs are only present during the non-breeding season and are difficult to locate on lower density islands (pers. obs.); therefore I chose not to include gravid females in my sampling regime.

\subsubsection{Sampling protocol}

Samples were collected at night (between 20:00 h and 04:00 h) from hand-captured individuals that had emerged from their underground burrows. To determine baseline CORT concentrations, a blood sample (up to $1 \mathrm{ml}$ ) was collected within 10 
min of capture from the base of the tail with a heparinized 23-gauge needle and $1 \mathrm{ml}$ syringe. In previous studies of tuatara, no significant effect of bleeding effort (time from capture to blood sample collection) was observed on baseline CORT concentrations in blood samples collected within 20 min of capture (Chapter Two; Tyrrell, 1993). After baseline samples were taken, individuals underwent $3 \mathrm{~h}$ capture-restraint in cloth capture bags either outdoors or in a research house (depending on the field site), whereupon a second blood sample (up to $1 \mathrm{ml}$ ) was taken.

Internal body temperature $\left(T_{\mathrm{b}}\right)$ was recorded with a cloacal thermocouple (Fluke ${ }^{\circledR}$ Multimeter, model: 179 , reported accuracy $\pm 1^{\circ} \mathrm{C}$ ) prior to taking blood samples (both baseline CORT and CORT response) for each individual, as $T_{\mathrm{b}}$ has been shown to influence CORT secretion in tuatara (Chapter Three). After the $3 \mathrm{~h}$ blood sampling was completed, individuals were weighed with a $1000 \mathrm{~g}$ spring scale (Pesola AG, Switzerland) to determine mass (g), and snout-vent length (SVL) (mm), tail length $(\mathrm{mm})$, and tail regeneration length $(\mathrm{mm})$ were measured with a ruler to determine body condition. Depending on field conditions, blood samples were separated either by centrifuge ( $5 \mathrm{~min}$ at $2000 \mathrm{rpm}$ ) or under normal gravity for 6 to $8 \mathrm{~h}$ at $4^{\circ} \mathrm{C}$ (Reimers et al., 1983; Sheriff et al., 2011). Plasma was transferred into cryogenic vials with a micropipette, stored in a cryogenic dry shipper at $-190^{\circ} \mathrm{C}$ (Thermo Scientific ${ }^{\mathrm{TM}}$, Arctic Express ${ }^{\mathrm{TM}}$ Dual 10) or in a freezer at $-20^{\circ} \mathrm{C}$ until return to the laboratory, and then stored at $-80^{\circ} \mathrm{C}$ until assayed.

\subsubsection{Linear ecological predictors: Principal components analysis (PCA) of ecological attributes}

I used principal components analysis (PCA) to identify combinations of ecological attributes that explain variation between island populations. A correlation matrix was used to generate PCA scores from ecological attributes (Table 4.1). Principal component axes with eigenvalues $\geq 1.0$ were retained and a minimum absolute value of $\geq 0.4$ for loading coefficients was chosen as a requirement for significance of an ecological variable in each PCA axis (Field et al., 2012). 
Table 4.1: Description and ecological attributes of study populations

\begin{tabular}{|c|c|c|c|c|c|c|c|c|c|}
\hline Population & Latitude & $\begin{array}{l}\text { Island } \\
\text { size } \\
\text { (ha) }\end{array}$ & $\begin{array}{r}\text { Tuatara } \\
\text { density } \\
\text { (\#/ha) }\end{array}$ & $\begin{array}{l}\text { Relative } \\
\text { seabird } \\
\text { density }\end{array}$ & $\begin{array}{l}\text { History of } \\
\text { introduced } \\
\text { rats }\end{array}$ & $\begin{array}{l}\text { Genetic } \\
\text { Diversity } \\
\text { (H) }\end{array}$ & $\begin{array}{c}\text { Sex } \\
\text { ratio } \\
(M: F)\end{array}$ & $\begin{array}{c}\text { Female } \\
\text { gravidity } \\
\text { rate } \\
(\% / y r)\end{array}$ & References \\
\hline Stephens & $40^{\circ} 40^{\prime} \mathrm{S}$ & 150 & 674 & high & None & 0.6 & $1: 1$ & 21.9 & $\begin{array}{l}\text { (Cree, 2014; Gaze, 2001; Hay et al., 2010; } \\
\text { Moore et al., 2010; Newman, 1987; Newman } \\
\text { et al., 1994) }\end{array}$ \\
\hline $\begin{array}{l}\text { North } \\
\text { Brother }\end{array}$ & $41^{\circ} 6^{\prime} \mathrm{S}$ & 4 & $\begin{array}{c}134- \\
159\end{array}$ & high & None & 0.1 & $\begin{array}{c}3.2: \\
1\end{array}$ & 10.8 & $\begin{array}{l}\text { (Cree, 2014; Cree et al., 1991a; Gaston and } \\
\text { Scofield, 1995; Gaze, 2001; Grayson et al., } \\
\text { 2014; Hay et al., 2010; Mitchell et al., 2010; } \\
\text { Nelson et al., 2002; Wilson, 2010) }\end{array}$ \\
\hline Lady Alice & $35^{\circ} 53^{\prime} \mathrm{S}$ & 155 & $\begin{array}{c}13.6- \\
17.7\end{array}$ & med & $\begin{array}{l}\text { Rats } \\
\text { eradicated } \\
\text { in } 1994\end{array}$ & 0.7 & $1: 1$ & $28.8-32$ & $\begin{array}{l}\text { (Cassey and Ussher, 1999; Cree, 2014; Cree } \\
\text { et al., 1995; Gaze, 2001; Hay et al., 2010; } \\
\text { Newman et al., 1994; Towns et al., 2007; } \\
\text { Tyrrell et al., 2000) }\end{array}$ \\
\hline Taranga & $35^{\circ} 58^{\prime} \mathrm{S}$ & 500 & 1.1 & low & $\begin{array}{l}\text { Rats } \\
\text { eradicated } \\
\text { in } 2011\end{array}$ & 0.7 & $\begin{array}{l}1: \\
1.5\end{array}$ & 27 & $\begin{array}{l}\text { (Buxton et al., 2013; Cree, 2014; Cree et al., } \\
\text { 1995; Gaze, 2001; Hay et al., 2010) }\end{array}$ \\
\hline
\end{tabular}




\subsubsection{Reproductive activity: Testosterone (T) secretion}

I determined plasma testosterone (T) concentrations (at both $0 \mathrm{~h}$ and $3 \mathrm{~h}$ ) in a subset of plasma samples obtained in this study from the Stephens (males: $n=10$, females: $n=20$ ) and North Brother (males: $n=15$, females: $n=15$ ) populations in Cook Strait. Plasma T concentrations can be used as a gauge for reproductive activity in males, where increased $\mathrm{T}$ implies increased sperm production, territoriality, courtship, and mating events (Cree et al., 1992; Gillingham et al., 1995). In females, plasma T concentrations are normally lower than males; however, results can be used to gauge percentage of reproductively active females in each population, as elevated T concentrations (obtained within $3 \mathrm{~h}$ of capture) in February-March distinguish female tuatara in ovulatory condition (Cree et al., 1991b).

\subsubsection{Enzyme-Immunoassay}

\subsubsection{Corticosterone}

Plasma CORT concentrations for all samples were determined as described in Chapter Two. Mean extraction efficiency of tritiated CORT $\left(\mathrm{H}^{3}\right)$ was $106 \% \pm 5 \%$ s.d. ( $n=25$ extractions) with an overall CV of 5.18\%. The mean intra-assay CV was $9.9 \%$ and the mean inter-assay CV was $15.4 \%$.

\subsubsection{Testosterone}

Plasma samples from the Cook Strait populations (Stephens and North Brother Island - NBI) obtained during the March 2012 breeding season ( $n=120)$ were sorted into male and female samples (30/plate) and extracted for assay as described for CORT in Chapter Two. Serial dilutions at 100\%, 80\%, 60\%, 40\%, 20\%, 10\%, 5\% and $1 \%$ of pooled male tuatara plasma samples showed good parallelism to the standard curve for testosterone $(\mathrm{T})$ over the assay standard range $(P>0.05)$. Samples were reconstituted with $2 \mathrm{ml}$ EIA buffer (for a 200 -fold dilution), vortexed and held at $4^{\circ} \mathrm{C}$ until assayed. 
Extraction efficiency was measured by comparing mean recovery of extracted $(n=5)$ versus non-extracted $(n=5) 2000 \mathrm{cpm}$ tritiated T [1,2,6,7- $\left.\mathrm{H}^{3}\right]$. Extracted plasma samples $(50 \mu \mathrm{l})$ were analysed in duplicate using commercial enzyme immunoassay kits (Cayman Chemical Co., Ann Arbor, MI) containing 96-well plates coated with mouse monoclonal anti-rabbit IgG antibody. Testosterone-specific acetylcholinesterase (AChE) tracer and sheep T antiserum were added to sample wells and placed on an orbital shaker for $2 \mathrm{~h}$. Plates were washed five times, developed for $1 \mathrm{~h}$ on an orbital shaker and subsequently read at $412 \mathrm{~nm}$. The concentration of $\mathrm{T}$ was calculated by comparing results to a standard curve; all samples yielded $\mathrm{T}$ concentrations within the $20-80 \%$ bound range. Mean extraction efficiency of $\left[1,2,6,7-\mathrm{H}^{3}\right]$ was $100 \% \pm 5 \%$ s.d. ( $n=4$ extractions) with an overall CV of $4.9 \%$. The mean intra-assay CV was $10.9 \%$ and the mean inter-assay CV was $12.4 \%$.

\subsubsection{Statistical analyses}

Data analyses were carried out using R v3.2.0 statistical software (R Development Core 2013) and Prism 6 (Graphpad Software Inc.). All data were checked for assumptions of normality and were transformed if necessary. To generate body condition scores, I calculated tail-corrected mass (TCM) following Newman et al. (1994) to account for tail-loss and regeneration, pooled data across both seasons (separated by sex) then generated standardized residuals from a regression of logTCM vs. logSVL (Schulte-Hostedde et al., 2005).

Linear models (LM) and linear mixed effects regression models (LMER) were fitted using the 'Ime4' package (Bates, 2013) to analyse: 1) inter-population variation in CORT secretion, body condition and T secretion, and 2) linear ecological predictors (PCA axes) of CORT secretion. Final models were selected through forward selection by comparing the addition of fixed and random effects to intercept-only baseline models (Field et al., 2012), whereupon parameter estimates were obtained with the summary() function in R. The 'ImerTest' package (Kuznetsova, 2013) was used to compute $P$-values for parameter estimates and statistical significance was assumed at $P<0.05$. 
First, I determined variation in CORT secretion and body condition among populations during the non-breeding and breeding seasons. Sex was not a significant predictor in any of the final models $(P>0.05)$; therefore sexes were pooled for all analyses. Final models for CORT secretion included response variables of log transformed baseline CORT and the CORT response ( $3 \mathrm{~h}$ CORT - $0 \mathrm{~h}$ CORT) and input variables of season (non-breeding, breeding), population (Stephens, NBI, LA, Taranga), linear covariates of internal body temperature $\left(T_{\mathrm{b}}\right)$ and body condition, and an interaction term of season* population. The final model for body condition included a response variable of body condition score and input variables of season (non-breeding, breeding), population (Stephens, NBI, LA, Taranga) and an interaction term of season* population.

Second, I examined linear ecological predictors (PCA axes) of CORT secretion through principle component regression models. Sex was not a significant predictor in any of the final models $(P>0.05)$; therefore sexes were pooled for all analyses. The final models included a response variable of log transformed CORT response $(3 \mathrm{~h}$ CORT - 0 h CORT) and input variables of season (non-breeding, breeding), linear covariates of PCA Axes (PC1 and PC2) and an interaction terms of season* PCA axes (PC1 and PC2).

Lastly, I determined variation in T secretion between a subset of two populations and examined associations with CORT secretion. The final model for T secretion included a response variable of $\mathrm{T}(\mathrm{ng} / \mathrm{ml})$ and input variables of hour $(0 \mathrm{~h}, 3 \mathrm{~h})$, population (Stephens, NBI), sex (M, F) and an interaction term of population * sex. The final model for CORT and T association included a response variable of log transformed CORT and input variables of hour $(0 \mathrm{~h}, 3 \mathrm{~h})$, population (Stephens, NBI), sex $(\mathrm{M}, \mathrm{F})$ and a linear covariate of T. A random effect of tuatara ID was included in both models to account for repeat sampling of individuals (at 0 and $3 \mathrm{~h}$ ). 


\subsection{Results}

\subsubsection{CORT secretion and body condition}

Baseline CORT did not significantly vary among populations during either the nonbreeding (Fig 4.1a, Table 4.2) or breeding (Fig 4.1a, Table 4.2) seasons. Baseline CORT secretion significantly increased from the non-breeding season to the breeding season in the Stephens (LM, $t=3.16, P=0.001$, Fig 4.1a) and LA (LM, $t=2.92$, $P=0.004$, Fig 4.1a) populations, but was similar between seasons in the NBI (LM, $t=$ 1.09, $P=0.277$, Fig 4.1a) and Taranga (LM, $t=-0.16, P=0.874$, Fig 4.1a) populations.

The CORT response varied significantly among populations during both seasons (Fig 4.1b, Table 4.2). During the non-breeding season, the CORT response was similar between the Stephens and NBI populations (Fig 4.1b, Table 4.2), the Taranga population was significantly higher than the Stephens (Fig 4.1b, Table 4.2) and NBI (LM, $t=3.55, P=0.001$, Fig $4.1 \mathrm{~b}$ ) populations, and the LA population was significantly higher than the Stephens (Fig 4.1b, Table 4.2), NBI (LM, $t=5.84, P<0.001$, Fig 4.1b), and Taranga (LM, $t=2.79, P=0.006$, Fig $4.1 \mathrm{~b}$ ) populations . During the breeding season, the CORT response was similar among the Stephens, LA, and Taranga populations, and was significantly lower in the NBI population (Fig 4.1b, Table 4.2). The CORT response significantly decreased from the non-breeding season to the breeding season in the LA population only (LM, $t=-4.38, P<0.001$, Fig $4.1 \mathrm{~b}$ ), but was similar between seasons in the Stephens (Fig 4.1b, Table 4.2), NBI (LM, $t=-1.27$, $P=0.205$, Fig $4.1 \mathrm{~b}$ ), and Taranga (LM, $t=-1.07, P=0.287$, Fig $4.1 \mathrm{~b}$ ) populations.

Body condition varied significantly among populations during both seasons (Fig 4.1c, Table 4.2). During the non-breeding season, body condition was significantly higher in the Stephens population compared to all other populations (Fig 4.1c, Table 4.2), was similar between the NBI and Taranga (LM, $t=1.14, P=0.256$, Fig 4.1c) populations, and the LA and Taranga (LM, $t=-1.10, P=0.270$, Fig 4.1c) populations, and was significantly lower in the NBI population compared to the LA population (LM, $t=-2.23, P=0.027$, Fig 4.1c). 
a) Baseline CORT (O h)

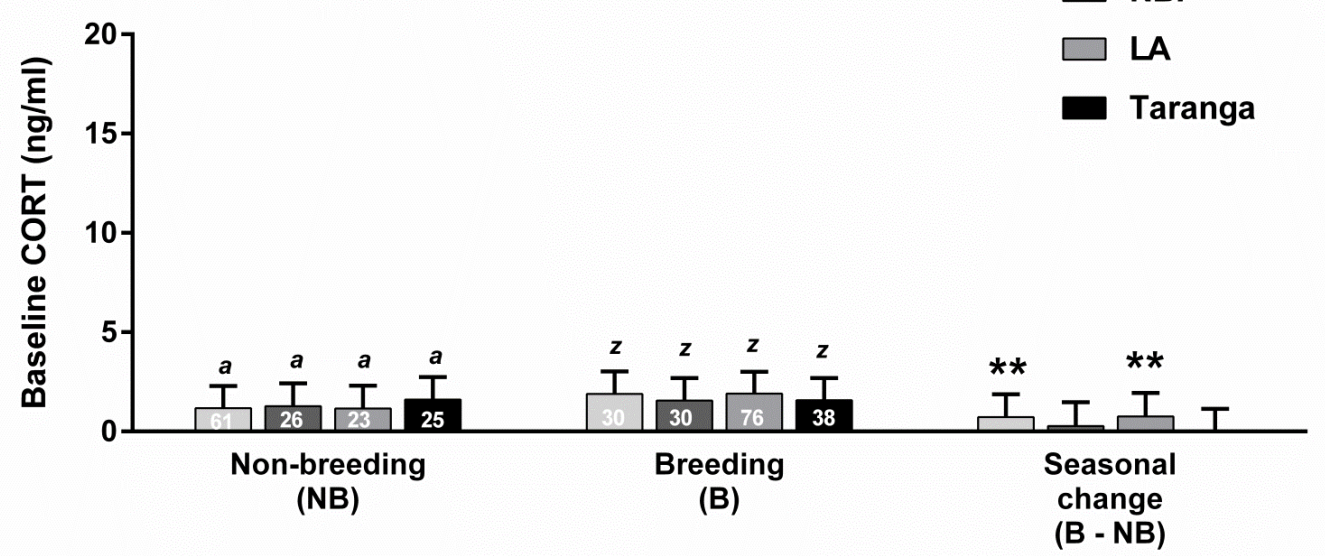

b) $3 \mathrm{~h}$ CORT response

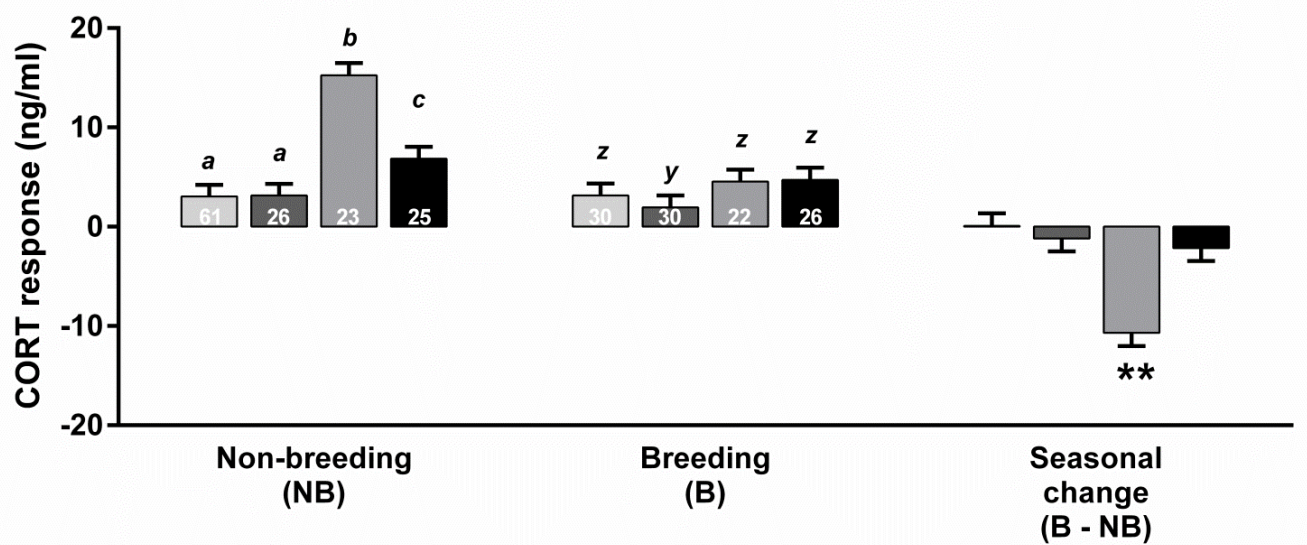

c) Body condition

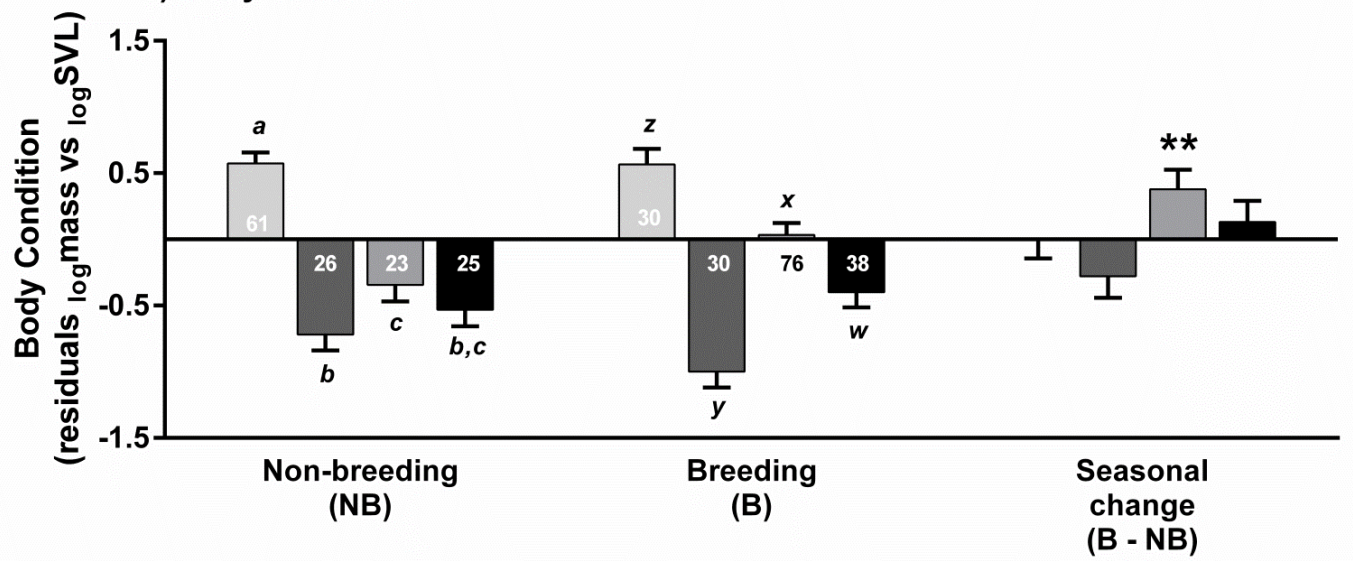

Figure 4.1: Patterns of a) baseline CORT, b) the CORT response ( $3 \mathrm{~h}-0 \mathrm{~h}$ CORT), and c) body condition (standardised residuals from regression of TCMlog/SVLlog) among four contrasting populations of adult tuatara (Sphenodon punctatus) during the non-breeding and breeding seasons. Data (Mean $\pm \mathrm{SE}$ ) from males and females (non-gravid only) from each population are pooled. Populations sharing the same letters are not significantly different during the nonbreeding $(a, b, c)$ and breeding $(z, y, x, w)$ seasons. Significant seasonal change (breeding season - non-breeding season) is indicated by asterisks $(P<0.01)$. Sample size $(n)$ is indicated by numbers at base of each bar. 
Table 4.2: Linear model parameter estimates for baseline CORT, the CORT response, and body condition examining variation among four populations of tuatara (Sphenodon punctatus): Stephens Island (ST), North Brother Island (NBI), Lady Alice Island (LA), and Taranga Island. ${ }^{1}$ Centred covariates of body temperature and body condition were included in CORT secretion models.

\begin{tabular}{|c|c|c|c|c|c|c|c|c|c|}
\hline \multirow[b]{2}{*}{ Parameter } & \multicolumn{3}{|c|}{ Baseline CORT¹ } & \multicolumn{3}{|c|}{ CORT response $^{1}$} & \multicolumn{3}{|c|}{ Body Condition } \\
\hline & Est $\pm s e$ & $t$ & $P$ & $E s t \pm s e$ & $t$ & $P$ & $E s t \pm s e$ & $t$ & $P$ \\
\hline \multicolumn{10}{|l|}{ non-breeding } \\
\hline (ST - Intercept) & $0.06 \pm 0.05$ & 1.320 & 0.187 & $0.54 \pm 0.07$ & 7.781 & $<0.001$ & $0.57 \pm 0.08$ & 6.891 & $<0.001$ \\
\hline season & $0.20 \pm 0.07$ & 3.162 & $<0.001^{* * *}$ & $0.01 \pm 0.10$ & 0.079 & 0.936 & $-0.01 \pm 0.13$ & -0.053 & 0.957 \\
\hline NBI & $0.03 \pm 0.07$ & 0.561 & 0.575 & $0.01 \pm 0.10$ & -0.682 & 0.496 & $-1.29 \pm 0.13$ & -9.390 & $<0.001^{* * *}$ \\
\hline LA & $-0.01 \pm 0.08$ & -0.070 & 0.944 & $0.69 \pm 0.12$ & 4.857 & $<0.001^{* * *}$ & $-0.91 \pm 0.14$ & -6.363 & $<0.001^{* * *}$ \\
\hline Taranga & $0.13 \pm 0.07$ & 1.814 & 0.070 & $0.35 \pm 0.09$ & 2.957 & $0.003^{*}$ & $-1.10 \pm 0.13$ & -7.920 & $<0.001^{* * *}$ \\
\hline \multicolumn{10}{|l|}{ breeding } \\
\hline (ST - Intercept) & $0.27 \pm 0.06$ & 4.806 & $<0.001$ & $0.54 \pm 0.07$ & 7.700 & $<0.001$ & $0.57 \pm 0.11$ & 4.874 & $<0.001$ \\
\hline season & $-0.20 \pm 0.07$ & -3.162 & $0.001^{* * *}$ & $-0.01 \pm 0.10$ & -0.079 & 0.936 & $0.01 \pm 0.13$ & 0.053 & 0.957 \\
\hline NBI & $-0.08 \pm 0.08$ & -1.032 & 0.303 & $-0.21 \pm 0.12$ & -2.126 & $0.034^{*}$ & $-1.56 \pm 0.16$ & -9.749 & $<0.001^{* * *}$ \\
\hline LA & $0.01 \pm 0.08$ & 0.080 & 0.936 & $0.16 \pm 0.11$ & 1.120 & 0.263 & $-0.91 \pm 0.14$ & -3.771 & $<0.001^{* * *}$ \\
\hline Taranga & $-0.08 \pm 0.08$ & -0.959 & 0.338 & $0.17 \pm 0.12$ & 1.210 & 0.227 & $-0.96 \pm 0.14$ & -6.129 & $<0.001^{* * *}$ \\
\hline
\end{tabular}


During the breeding season, body condition varied significantly among all four populations (Fig 4.1c, Table 4.2) with highest to lowest body condition observed in the Stephens, LA, Taranga and NBI populations, respectively. Body condition significantly increased from the non-breeding season to the breeding season in the LA population only (LM, $t=2.55, P=0.011$, Fig $4.1 \mathrm{c}$ ), but was similar between seasons in the Stephens (Fig 4.1c, Table 4.2), NBI (LM, $t=-1.73, P=0.08$, Fig 4.1c), and Taranga (LM, $t=0.83, P=409$, Fig $4.1 \mathrm{c}$ ) populations.

\subsubsection{Linear ecological predictors of CORT secretion (PCA axes)}

I used principal components analysis (PCA) to simplify five ecological variables into linear ecological predictors (Table 4.3). Together, the first two principal components (PC1, PC2) explained approximately $96 \%$ of the variance in ecological attributes among the four island populations (Table 4.3).

Table 4.3: Results from principal components analysis (PCA) of five ecological variables from four island populations of tuatara (Sphenodon punctatus). Coefficient loadings in bold ( $\geq$ absolute value of 0.4 ) are interpreted as significant.

\begin{tabular}{lcc}
\hline \multirow{2}{*}{ Ecological Attribute } & \multicolumn{2}{c}{ Coefficient loadings } \\
\cline { 2 - 3 } & PC1 & PC2 \\
\hline Latitude (deg, min) & $\mathbf{0 . 4 5 0}$ & -0.142 \\
Tuatara density (\#/ha) & $\mathbf{0 . 4 0 0}$ & $\mathbf{0 . 4 4 0}$ \\
Seabird abundance (\#/ha) & $\mathbf{0 . 4 7 6}$ & -0.120 \\
Genetic diversity (H) & 0.319 & $\mathbf{0 . 5 8 3}$ \\
Sex ratio (\% males) & 0.284 & $\mathbf{- 0 . 6 4 5}$ \\
\hline Importance of components & & \\
\hline Eigenvalues & 2.06 & 1.24 \\
Proportion of variance & $70 \%$ & $26 \%$ \\
Cumulative proportion & $70 \%$ & $96 \%$ \\
\hline
\end{tabular}

In general, the PC1 axis (explaining 70\% variance) distinguished an ecological predictor based on habitat variation, in which individuals that loaded positively on 
the PC1 axis were from populations at higher latitudes combined with higher tuatara densities and higher seabird abundance (Table 4.3). The PC2 axis (explaining 26\% variance) teased out an ecological predictor based on demogenetic variation, in which individuals that loaded positively on the PC2 axis were from populations with a lower percentage of males combined with higher genetic diversity (Table 4.3).

Table 4.4 presents results from the principle component regression analyses of ecological predictors. A significant relationship between baseline CORT and PC1 or PC2 scores was not observed in either season ( $L M, P>0.05$, Figs 4.2a and 4.3a). However, a significant negative relationship was observed between the CORT response and PC1 scores during both the non-breeding season (Fig 4.2b, Table 4.4) and (to a greater extent) the breeding season (Fig 4.2b, Table 4.4), and a significant positive relationship was observed between the CORT response and PC2 scores during the breeding season (Fig 4.3b, Table 4.4) but not during the non-breeding season (Fig 4.3b, Table 4.4). In other words, higher CORT responses are associated with a) lower latitude, lower tuatara density and lower seabird abundance (both seasons), and b) balanced sex ratios and greater genetic diversity (breeding season only).

Table 4.4: Linear model parameter estimates for the CORT response in tuatara (Sphenodon punctatus) with a fixed effect of season (non-breeding, breeding), and linear covariates of PC1 scores (latitude, tuatara density, seabird abundance) and PC2 scores (\% males, genetic diversity) as predictor variables.

\begin{tabular}{lrcc}
\hline Parameter & Est $\pm \boldsymbol{s} \boldsymbol{e}$ & $\boldsymbol{t}$ & $\boldsymbol{P}$ \\
\hline (Intercept) & $0.78 \pm 0.03$ & 27.041 & $<0.001^{* * *}$ \\
season & $-0.04 \pm 0.04$ & -0.899 & 0.367 \\
PC1 & $-0.13 \pm 0.01$ & -9.300 & $<0.001^{* * *}$ \\
season x PC1 & $0.07 \pm 0.02$ & 3.101 & $0.002^{* *}$ \\
& & & \\
(Intercept) & $0.73 \pm 0.03$ & 22.194 & $<0.001^{* * *}$ \\
season & $0.03 \pm 0.05$ & 0.639 & 0.523 \\
PC2 & $-0.01 \pm 0.03$ & -0.542 & 0.588 \\
season x PC2 & $0.13 \pm 0.04$ & 3.581 & $<0.001^{* * *}$ \\
\hline
\end{tabular}




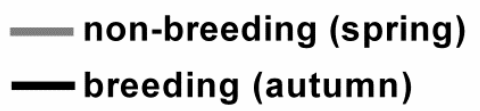

a) Baseline CORT

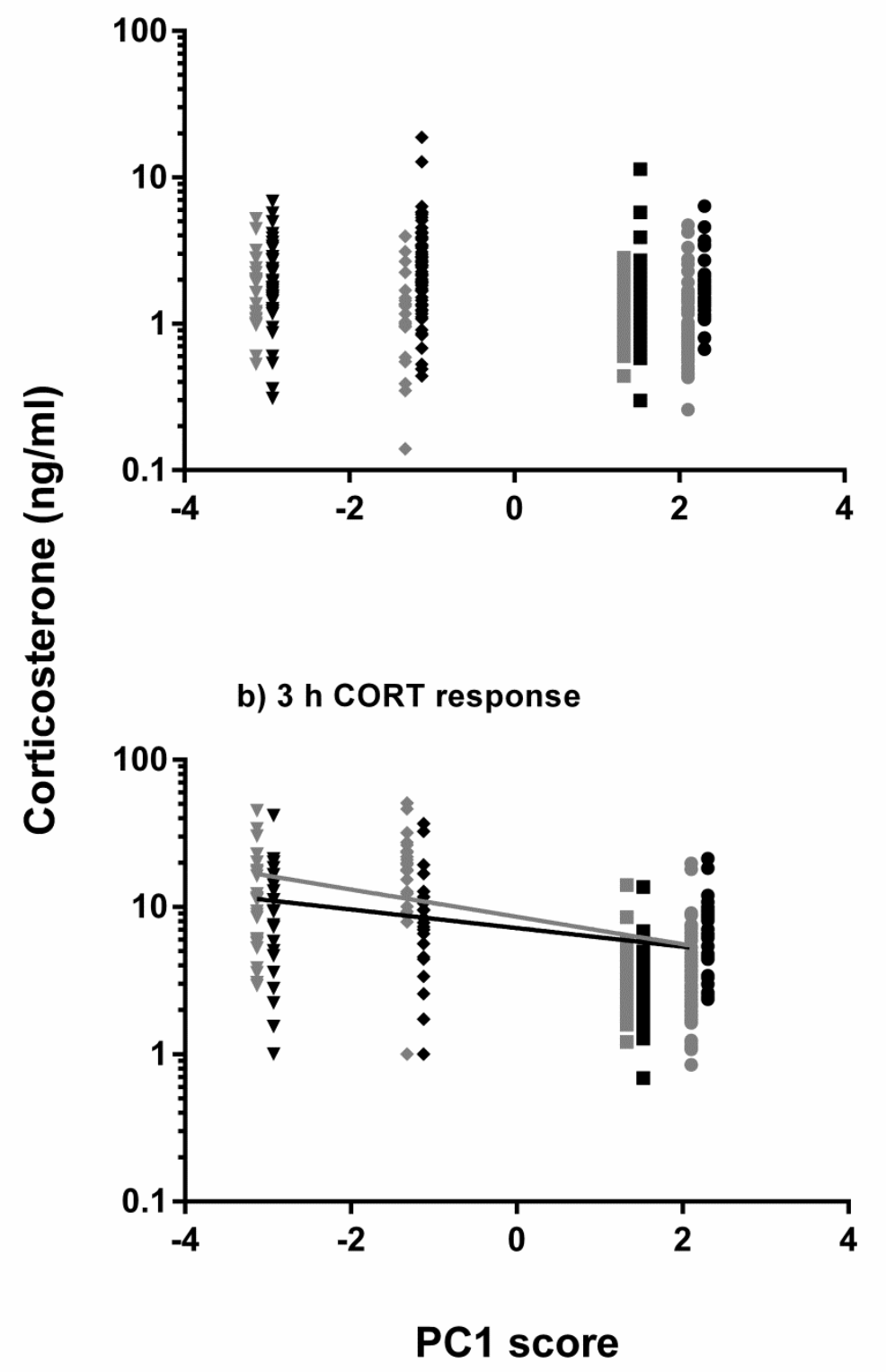

Figure 4.2: The relationship between PC1 "latitude, tuatara density and seabird abundance" on a) baseline CORT and $b$ ) the CORT response in four populations of tuatara (Sphenodon punctatus) during the non-breeding (gray symbols) and breeding (black symbols) seasons. Individuals with higher PC1 scores are from populations at higher latitudes with higher tuatara density and seabird abundance. A significant relationship was not observed between baseline CORT and PC1 scores. However, a significant negative relationship was observed between the CORT response and PC1 scores during the breeding season (indicated by the solid black line) and to a greater extent during the non-breeding season (indicated by the solid gray line). 


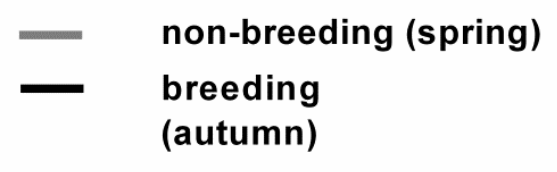

- Stephens

- North Brother

- Lady Alice

v Taranga

a) Baseline CORT

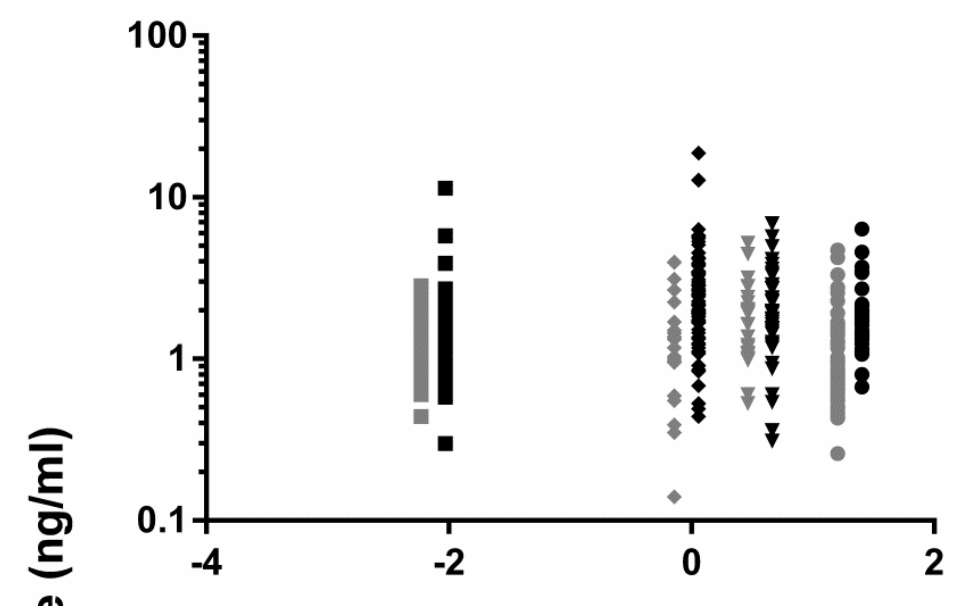

b) $3 \mathrm{~h}$ CORT response

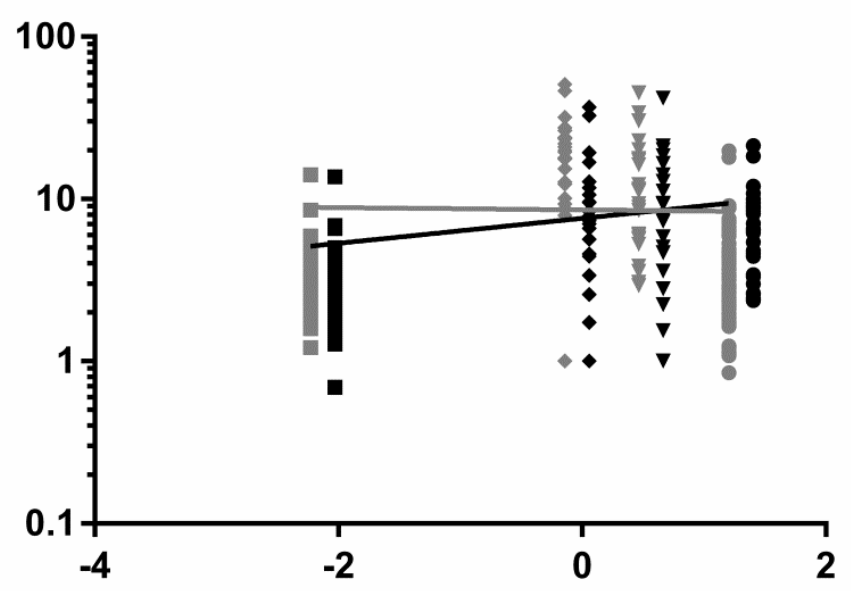

PC2 score

Figure 4.3: The relationship between PC2 "percentage of males in population and genetic diversity" on a) baseline CORT and b) the CORT response in four populations of tuatara (Sphenodon punctatus) during the non-breeding (gray symbols) and breeding (black symbols) seasons. Individuals with higher PC2 scores are from populations with higher tuatara density, a lower percentage of males and higher genetic diversity $(H)$. A significant relationship was not observed between baseline CORT and PC2 scores. However, a significant positive relationship was observed between the CORT response and PC2 scores during breeding season (indicated by the solid black line) but not during the non-breeding season (indicated by the solid gray line) 


\subsubsection{Reproductive activity: Testosterone (T) secretion during the breeding season}

Testosterone (T) secretion in males varied between the two populations sampled during the breeding season, with males from the Stephens population showing significantly higher $\mathrm{T}$ secretion (almost two-fold) compared to males from the NBI population (LMER, $t=2.53, P=0.013$, Fig 4.4). Testosterone secretion in females was similar between populations (LMER, $t=0.65, P=0.517$, Fig 4.4). A significant difference in $\mathrm{T}$ was observed between sexes in the Stephens population only, with males showing higher T secretion than females (LMER, $t=3.83, P<0.001$, Fig 4.4 ). Surprisingly, males and females in the NBI population had similar T secretion (LMER, $t=1.87, P=0.065$, Fig 4.4). I did not observe a significant T response to $3 \mathrm{~h}$ capture-restraint in the Stephens (LMER, $t=0.69, P=0.496$ ) or NBI population (LMER, $t=-0.37, P=0.713$ ), nor did I find a significant linear relationship between CORT secretion (baseline and/or CORT response) and T secretion (LMER, $t=0.33$, $P=0.743$ ) during the breeding season.
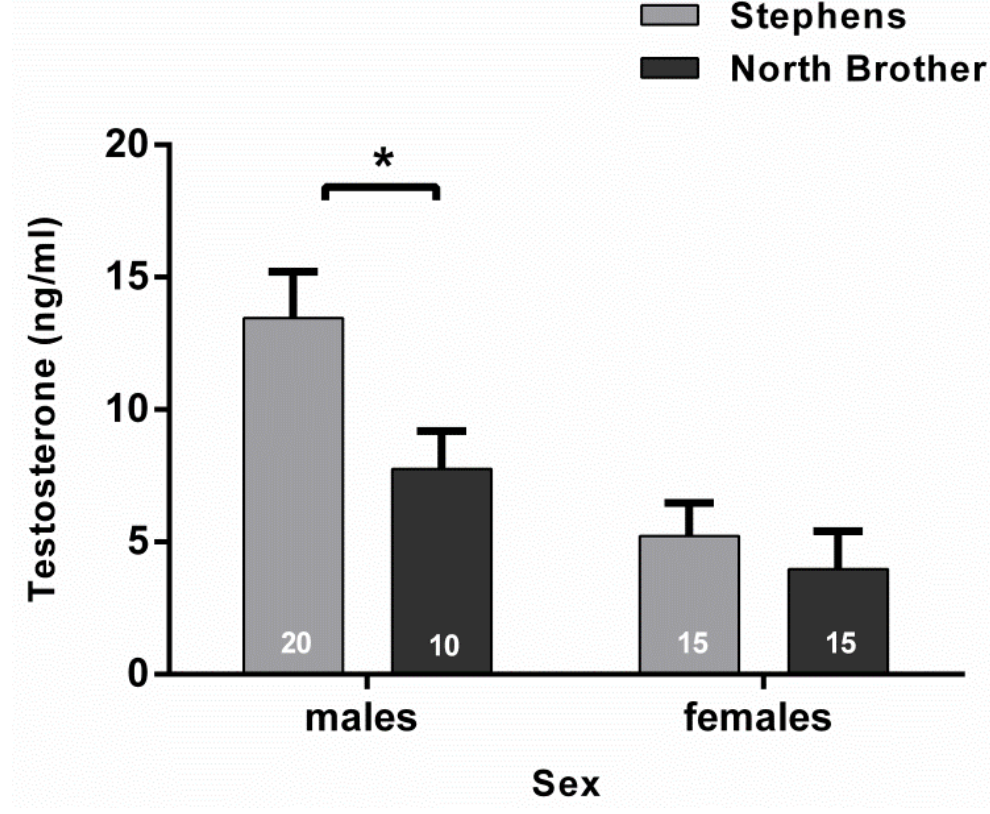

Figure 4.4: Baseline testosterone secretion (mean \pm SE) in male and female tuatara (Sphenodon punctatus) from Stephens and North Brother populations during the breeding season (autumn). A significant difference between populations is indicated by an asterisk (males only, $P=0.013$ ). Sample size $(n)$ is indicated by numbers at base of each bar. 


\subsection{Discussion}

I examined variation in CORT secretion among four populations of a rare reptile the tuatara, Sphenodon punctatus) during the non-breeding and breeding seasons and tested linear ecological predictors of CORT secretion (results summarised in Table 4.5). I established that baseline CORT is similar, whereas the CORT response varies, among populations during each season. Furthermore, I show significant relationships between linear ecological predictors (PCA axes) ant the CORT response but not baseline CORT, suggesting that habitat factors (PC1) and demogenetic factors (PC2) can influence the CORT response. These findings show that the CORT response appears more sensitive than baseline CORT to ecological factors in tuatara, and thus may be a more informative gauge to assess and/or monitor populations.

Secondly, I examined variation in testosterone (T) concentrations between a subset of two populations during the breeding season and examined the association between reproductive activity and seasonal modulation of baseline CORT. I did not find a significant linear relationship between T and CORT secretion during the breeding season; however, significant inter-population variation in T secretion was observed in males, but not females, and may indicate a positive association between male reproductive activity (as indicated by T concentrations) and baseline CORT increase from the non-breeding season to the breeding season. 
Table 4.5: Summary of results. (a) Inter-population comparisons for the non-breeding season, breeding season, and seasonal change. Relative levels of inter-population variation of baseline CORT, the CORT response, body condition, and reproductive activity $(\mathrm{T})$ among/between populations are shown. (b) Relationship/association between ecological factors and relevant response variables with direction of trend and associated season(s) shown.

\begin{tabular}{lcccc}
\hline & \multicolumn{3}{c}{ Inter-population variation } \\
\cline { 2 - 5 } $\begin{array}{l}\text { a) Inter-population } \\
\text { comparison }\end{array}$ & $\begin{array}{c}\text { Baseline } \\
\text { CORT }\end{array}$ & $\begin{array}{c}\text { CORT } \\
\text { Response }\end{array}$ & $\begin{array}{c}\text { Body } \\
\text { Condition }\end{array}$ & $\begin{array}{c}\text { Reproductive } \\
\text { Activity (T) }\end{array}$ \\
\hline $\begin{array}{l}\text { Non-breeding season } \\
\text { (NB) }\end{array}$ & low & high & high & - \\
$\begin{array}{l}\text { Breeding } \\
\text { season } \\
\text { (B) }\end{array}$ & low & low & high & $\begin{array}{c}\text { high (males) } \\
\text { low(females) }\end{array}$ \\
$\begin{array}{l}\text { Seasonal modulation } \\
\text { (NB to B) }\end{array}$ & & & & \\
\end{tabular}

\section{b) Relationship/} association

Body Condition

Seasonal modulation

Habitat effects (PC1)

$\uparrow$ Latitude

$\uparrow$ Tuatara density

$\uparrow$ Seabird abundance

\section{Response} variable

\section{Direction}

Season

CORT response relationship (NB \& B)

Baseline CORT Positive NB to B

$\begin{array}{lc}\text { CORT response } \quad \text { Negative } & \text { Both } \\ \text { (NB \& B) }\end{array}$

CORT response Positive

B

$\downarrow$ Male-bias sex ratio

$\uparrow$ Genetic diversity

Male reproductive activity ${ }^{1}$

$\uparrow \mathrm{T}$ concentrations

Seasonal change
(B-NB)

in baseline CORT

Positive

B

${ }^{1}$ Stephens and North Brother populations only 


\subsubsection{The CORT response and body condition vary among populations, but baseline CORT does not}

Body condition varied significantly among populations; however, body condition was not a significant predictor of CORT secretion (baseline CORT or the CORT response) in spite of the apparent variation. The lack of relationship may be explained by the fact that a certain threshold must be met for body condition to affect CORT secretion (Romero and Wikelski, 2001).

Contrary to my prediction, baseline CORT did not vary among populations during the non-breeding or breeding seasons. Baseline CORT is often used as an indicator of overall health or stress levels (Bonier et al., 2009) and the CORT response is often used as an indicator of an individual's ability to respond to a stressor (Breuner et al., 2008). The CORT response varied significantly among populations during both seasons. During the non-breeding season, the CORT response was higher in the Lady Alice and Taranga (Northern) populations, compared to the Stephens and North Brother (Cook Strait) populations, which is consistent with my prediction. However, the CORT response was similar between all populations during the breeding season, except for the North Brother population which was significantly lower. My findings show that the CORT response appears more sensitive than baseline CORT to ecological factors in tuatara, and thus may be a more informative gauge to assess and/or monitor populations.

\subsubsection{Relationships between The CORT response and linear ecological attributes (PC1 and PC2 scores)}

Significant relationships were observed between the CORT response, but not baseline CORT, and linear ecological attributes (PC1 and PC2). Thus, the variation in the CORT response that I observed among populations during the non-breeding and breeding seasons is currently best explained by 1) habitat factors of latitude, tuatara density and seabird abundance (PC1) and 2) demogenetic factors of sex ratio and genetic diversity (PC2). 


\subsubsection{Habitat factors of latitude, tuatara density and seabird abundance (PC1)}

A significant positive relationship was observed between PC1 scores and the CORT response during the non-breeding and breeding seasons. The first PCA axis (PC1) had significant positive loadings of latitude, tuatara density and seabird abundance (Table 4.3). In other words, higher PC1 scores are connected to individuals from populations at higher latitudes with higher tuatara density/seabird abundance.

It is possible that tuatara populations at lower latitudes mount a greater CORT response due to the milder climate (where mounting a greater CORT response may be less energetically costly). Warmer but drier climates (not necessarily more benign for tuatara) are experienced at lower latitudes, compared to higher latitudes. Moore et al. (2001) found that male garter snakes (Thamnophis sirtalis) living at a lower latitude had higher CORT responses than those at higher latitudes, and the authors suggest that snakes inhabiting milder climates (with extended breeding seasons) can energetically afford to mount a greater CORT response. Similarly, a recent study on tadpoles (Rana temporaria) found that the CORT response was higher in low-latitude populations, and the authors associated a lower CORT response in high-latitude populations with avoidance of CORT-mediated reduction in growth and development (Dahl et al., 2012). In birds, there is support that populations from higher latitudes exhibit a lower CORT response compared to those in lower latitudes (Hau et al., 2010; Silverin et al., 1997). On the other hand, there are also studies in both reptiles and birds that have found no correlation between latitude and the CORT response (Hews and Baniki, 2013; Quirici et al., 2014).

Lower tuatara density/seabird abundance is indicative of a history of introduced rats (the Pacific rat, Rattus exulans) (Cree et al., 1995; Jones et al., 2008; Towns, 2009) and it is possible that past/recent presence of rats has directed selection of a greater CORT response to deal with indirect and direct effects of predation and/or resource competition. Tyrrell et al. (2000) observed that tuatara from a ratinhabited island had significantly higher CORT responses, but similar baseline CORT, compared to tuatara from a rat-free island (Blair et al., 2000). Similarly, higher CORT responses have been observed in marine iguanas (Amblyrhynchus cristatus) 
exposed to invasive predatory mammals (Felis catus, Canis lupus) (Berger et al., 2007; Rödl et al., 2007). Therefore, the past/recent presence of an introduced species/predator may induce a long-term effect of sensitisation in stress responsiveness and/or a higher CORT response may be a modulating mechanism to cope with impact of a novel stressor (Berger et al., 2007; Langkilde and Trompeter, 2011; Rödl et al., 2007).

The significant relationship between PC1 and the CORT response was stronger during the non-breeding season (Fig 4.2b), which could indicate that competition for food resources may be greater during the non-breeding season, especially on islands with a history of rats. There is ample evidence that seabirds elevate invertebrate density, and both invertebrates and seabirds (which can be reduced by introduced rats) are an important food source for tuatara in island populations (Markwell and Daugherty, 2002; Towns, 2009; Towns and Broome, 2003; Towns et al., 2007). While I did not collect any quantitative measures of food availability, the seasonal change in body condition observed in the Lady Alice population in this study (in which body condition significantly increased from the non-breeding to the breeding season) provides support for reduced food availability during the non-breeding season.

\subsubsection{Demogenetic factors of sex ratio and genetic diversity (PC2)}

A significant negative relationship was observed between PC2 scores and the CORT response during the breeding season, but not during the non-breeding season. . The second PCA axis (PC2) had a significant negative loading of percentage of males in the population and a significant positive loading of genetic diversity (Table 4.3). In other words, higher PC2 scores are connected to individuals from populations with a lower percentage of males present and higher genetic diversity.

A highly skewed male-biased sex ratio in the North Brother population (Grayson et al., 2014) could indicate more male-male aggressive interactions during the breeding season; therefore, the CORT response may be modulated in tuatara to avoid chronic elevation of CORT. Male tree lizards (Urosaurus ornatus) experiencing 
a single male-male aggressive encounter experienced a sustained CORT response up to $24 \mathrm{~h}$, but males that continued interacting with other males had a dampened CORT response, which may facilitate metabolic recovery (Knapp and Moore, 1995). In male fruit bats (Artibeus jamaicensis), a dampened CORT response during the breeding season could reflect lowered stress sensitivity to avoid chronically elevated CORT levels in times of aggressive and costly male-male encounters (Klose et al., 2006).

There are very few studies examining relationships between genetic diversity and the CORT response, and I found none for reptiles or birds, however; my results are consistent with studies on mammals. Sea otter populations with lower genetic diversity had lower CORT response to capture (Larson et al., 2009). Sarrieau et al. (1998) found that genetic diversity had no effect on baseline CORT in rats; whereas, the CORT response was lower in inbred rats compared to outbred and hybrid rats. Clearly, further research examining relationships between demogenetic effects and CORT secretion in reptiles is required to determine whether consistent patterns are seen across species.

\subsubsection{Associations between reproductive activity (T) and modulation of CORT secretion}

A significant seasonal increase in baseline CORT (from the non-breeding season to the breeding season) was observed in only two of the four populations, which reveals that (contrary to my prediction) seasonal modulation of baseline CORT varies among populations. Seasonal change in baseline CORT was slight in the North Brother population and negligible in the Taranga population. Conversely, the Stephens and Lady Alice populations had significant seasonal change in baseline CORT, with increased secretion during the breeding season.

Numerous studies have reported increased baseline CORT in vertebrates during the breeding season, which is attributed to meeting energetic demands of reproductive activities (Moore et al., 2001; Moore et al., 2000b; Romero, 2002; Wingfield and Sapolsky, 2003). The CORT-fitness hypothesis suggests that the relationship 
between CORT secretion and fitness may depend on reproductive activity of individuals (Bonier et al., 2009) and that a seasonal increase in baseline CORT levels is necessary to meet demands of predictable reproductive activities such as gametogenesis, courtship and mating (Bonier et al., 2009; Landys et al., 2006; Whirledge and Cidlowski, 2013). Therefore, variation in seasonal modulation of baseline CORT among populations may be explained by different levels of reproductive activity. In support of this idea, I determined levels of T secretion (as a proxy of reproductive activity) in male and female tuatara from the Stephens population (significant seasonal change in baseline CORT) and the North Brother population (no seasonal change in baseline CORT) to examine the association between seasonal modulation of baseline CORT and reproductive activity.

Testosterone secretion in males from the Stephens population $(\sim 14 \mathrm{ng} / \mathrm{ml})$ was nearly twice the level of $\mathrm{T}$ secretion observed in males from the North Brother population ( $7.5 \mathrm{ng} / \mathrm{ml}$ ) (Fig 4.4). Previously reported levels of T concentrations in male tuatara are $~ 14-16 \mathrm{ng} / \mathrm{ml}$ on Stephens Island (Cree, 2014; Cree et al., 1990b) and $\sim 10 \mathrm{ng} / \mathrm{ml}$ on NBI (Cree, 2014). As expected, I found higher T secretion in males compared to females, but the difference only reached statistical significance in the Stephens population. It is generally recognized that territoriality and other aspects of male mating behaviour are regulated by androgens such as T (Garamszegi et al., 2005; Jessop et al., 1999a; Schramm et al., 1999; Sinervo and Miles, 2011; Wingfield et al., 1990). Given the highly male-biased sex ratio (3.2 males : 1 female) in the North Brother population (Grayson et al., 2014), it is probable that some males have entirely no access to females, as during the breeding season (in any one year) in the North Brother population, it is likely that only a few dozen females are in breeding condition (i.e. sexually receptive) (Mitchell et al., 2010). As male tuatara exhibit highly territorial behaviour (Moore, 2008), a proportion of males in the North Brother population are probably constrained to a 'male-only' environment, which may explain the lower levels of reproductive activity (specifically courting, mating, territorial defence and mate guarding). Research on reproductive activity and mating behaviour in tuatara is currently limited to information collected on Stephens Island; and to my knowledge there have been no studies that examine the association between baseline CORT and male reproductive activity, therefore 
studies on mating behaviour/reproductive activity in contrasting ecological scenarios would be required to support this association.

Testosterone secretion varied between populations for males, but not for females. Previous studies have shown that the annual gravidity rate for female tuatara from the North Brother population was half that of the Stephens population (Table 4.1). My results for $\mathrm{T}$ secretion in females suggest that the proportion of ovulating females is similar between the Stephens and North Brother populations. Possible explanations could be that female gravidity rates in the North Brother population have increased, that females ovulate but fail to produce shelled eggs, or that females nest earlier (and are no longer gravid when sampled). Alternatively, a sampling bias could have occurred in my study if ovulating (i.e. sexually receptive) females were more readily captured during the breeding season. Nonetheless, inter-population variation in $\mathrm{T}$ was observed in males (but not females) and was positively associated (though not statistically tested) with increased baseline CORT from the nonbreeding season to the breeding season, suggesting male reproductive activity may drive the seasonal change in baseline CORT.

\subsection{Conclusions}

Results from this study demonstrate that baseline CORT is relatively stable among populations, despite considerable environmental and genetic variation among populations. However, the CORT response varies and significant relationships between the CORT response and linear ecological predictor are observed, which suggests that stress reactivity is more sensitive than baseline CORT to ecological conditions (such as climate, food resource availability and/or introduced species) and/or demogenetic effects (such as sex ratio and/or inbreeding depression). Future research could tease out the influence of habitat and demogenetic predictors on the CORT response in tuatara, specifically through translocation programs, by examining whether patterns of the CORT response at the population-level are solely a product of selection and are not plastic to changes in the environment. 



\section{Moving house: Long-term dynamics of corticosterone secretion are unaltered in translocated populations of a rare reptile (the tuatara, Sphenodon punctatus) ${ }^{1}$}

\subsection{Abstract}

Translocations are an important conservation tool used to restore at-risk species to their historical range. Unavoidable procedures during translocations, such as habitat disturbance, capture, handling, processing, captivity, transport and release to a novel environment, have the potential to be stressful for most species. In this study, I examined acute and chronic stress (through the measurement of the glucocorticoid corticosterone - CORT) in a rare reptile (the tuatara, Sphenodon punctatus). Here, I found that 1 ) the acute CORT response remains elevated during the initial translocation process, but does not increase with cumulative stressors, and 2) the long-term dynamics of CORT secretion are similar in translocated and source populations. Taken together, my results show that translocated tuatara are generally resistant to cumulative acute stressors and show no hormonal sign of chronic stress. Translocation efforts in tuatara afford the potential to reduce extinction risk and restore natural ecosystems.

\footnotetext{
${ }^{1}$ This chapter is based on the following publication with minor modifications: Anderson L, Cree A, Towns D, Nelson (2015) Moving house: Long-term dynamics of corticosterone secretion are unaltered in translocated populations of a rare reptile (the tuatara, Sphenodon punctatus). Conservation Physiology
} 


\subsection{Introduction}

Translocations are human-assisted movements of living organisms from one area to another and are an important tool for conservation efforts and population restoration of species at risk (Armstrong and Seddon, 2008; Ewen et al., 2012; Seddon et al., 2014). The International Union for Conservation of Nature (IUCN) recognises two types of conservation translocation to restore populations, namely (i) reinforcements, in which individuals are released into an existing population of conspecifics to enhance the sustainability of populations, and (ii) reintroductions, in which individuals are released in a historically occupied area in order to re-establish a population after extirpation. Although these type of movements are ultimately aimed at helping species, the translocation process is inherently stressful, as associated procedures such as habitat disturbance, capture, handling, processing, captivity, transport and release to a novel environment are necessary and unavoidable (Dickens et al., 2010; Germano and Bishop, 2009; Parker et al., 2012). In a recent review, Tarszisz et al. (2014) identified physiology as a key disciplinary area that is lacking attention in conservation translocations, and also highlighted how physiological data can improve short- and long-term translocation success.

In vertebrates, the stress-response produces a rapid increase in glucocorticoid hormone secretion (corticosterone (CORT) or cortisol) to help individuals cope with immediate stressors (Wingfield et al., 1998); consequently, non-essential processes (such as reproduction and growth) are suspended until homeostasis returns. Although the stress response serves to promote immediate survival, prolonged or sustained CORT secretion (typically expected during translocations) can manifest as 'chronic stress', and is generally considered detrimental to overall health and fitness (i.e. the CORT-Fitness Hypothesis) (Almasi et al., 2013; Bonier et al., 2009; Parker et al., 2012; Sapolsky et al., 2000; Wingfield and Sapolsky, 2003). In a recent review of the associations between stress and movement of animals, Teixeira et al. (2007) concluded that stress is a contributing factor to the success or failure of a translocation project. Stress induced by the initial translocation process and relocation to a novel environment increases the vulnerability of individuals to reproductive failure, disease, starvation, predation, and long-range dispersal, 
thereby decreasing the chance that individuals will survive and that a self-sustaining population will result (Dickens et al., 2009; Dickens et al., 2010; Parker et al., 2012; Teixeira et al., 2007).

Measuring and monitoring CORT secretion is the most widely used method for assessing stress in vertebrates (Dickens et al., 2010; Sheriff et al., 2011; Wikelski and Cooke, 2006). Although several factors relevant to translocation efforts influence CORT secretion, studies that assess and monitor stress (by way of CORT secretion) throughout and after the translocation process are limited (Germano and Bishop, 2009; Harrington et al., 2013; Tarszisz et al., 2014). Numerous studies have shown that associated procedures commonly applied in translocation programs (such as capture, handling, transport) stimulate a significant stress-response and influence CORT secretion (Baker et al., 2013; Bliley and Woodley, 2012; Fazio and Ferlazzo, 2003; Fazio et al., 2014; Langkilde and Shine, 2006; Narayan and Hero, 2011). Similarly, altered CORT secretion has been associated with variation of environmental factors such as exposure to humans (French et al., 2010; Taylor et al., 2014) and novel predators (Berger et al., 2007; Rödl et al., 2007), change in food availability (Bryan et al., 2014; Kitaysky et al., 2007; Woodley et al., 2003), latitudinal differences (Dahl et al., 2012; Eikenaar et al., 2012; Quirici et al., 2014; Silverin et al., 1997) and habitat type (Bauer et al., 2013; French et al., 2008; Li et al., 2012; Zhang et al., 2011). In addition to experiencing acute stressors during the initial translocation process, translocated individuals released into new environments are faced with several survival challenges (such as finding food, shelter, and avoiding predators); therefore, physiological stress is inevitable (Teixeira et al., 2007).

Here, I examine the acute CORT response and long-term dynamics of CORT secretion through the translocation process in a rare reptile, the tuatara (Sphenodon punctatus). Tuatara are a protected reptile endemic to New Zealand and are the only living representatives of the reptilian order Rhynchocephalia (Jones and Cree, 2012). Although tuatara are now considered non-threatened but "at-risk - relict" (Hitchmough et al., 2013), translocations contribute to conservation and ecological restoration efforts and serve to re-establish tuatara within their pre-human range 
(Cree, 2014; Hitchmough et al., 2013). In addition to easing extinction pressure, translocations also offer a chance to examine and address relevant research questions (Cree, 2014; Germano and Bishop, 2009; Miller et al., 2012). In 2012, wild tuatara were translocated to six island and mainland sanctuaries from two source populations (Lady Alice Island and Stephens Island/Takapourewa, New Zealand) (Cree, 2014), which presented an excellent opportunity to examine the CORT response to translocation in multiple populations. Previous studies have examined patterns of CORT secretion in tuatara; in general, baseline CORT in tuatara is fairly low (with plasma concentrations typically $2-5 \mathrm{ng} / \mathrm{ml}$ ), a significant CORT response to capture-restraint is observed, and female reproductive condition, body temperature, and season (but not time of day) are influential factors (Chapter Two; Chapter Three, Chapter Four; Tyrrell and Cree, 1998). I found that baseline CORT was similar among all populations; however, the CORT response varied with latitude, seabird density, sex-ratio and genetic diversity (Chapter Four). Although translocations of tuatara continue to happen, CORT secretion (as an indicator of stress) during and after the translocation process has not been examined. Comparing CORT secretion simultaneously in translocated and source populations of tuatara would allow detection of altered CORT secretion that is correlated with environmental and/or habitat change and that would be an indication of chronic stress.

This study had two aims: first, I examined the acute CORT response in tuatara at different stages of the initial translocation process, and tested the prediction that the acute CORT response would be amplified with cumulative stressors. Second, I tested whether long-term changes in CORT secretion provide evidence of 'chronic stress' in three translocated populations (compared with the corresponding source populations as controls). I predicted that 1) baseline CORT (post-translocation) would be similar among all populations and 2) the CORT response (posttranslocation) would be amplified in translocated populations that experienced a marked environmental and/or habitat change (e.g. a greater latitudinal shift). Moreover, body condition indices (mass relative to snout-vent length) in translocated populations (along with source populations as controls) were examined, as chronic stress can influence energy expenditure (Romero, 2002). 


\subsection{Materials and methods}

\subsubsection{Study design}

I took advantage of two planned translocations in New Zealand (Fig 5.1) to examine short-term and long-term dynamics of CORT secretion in tuatara throughout the translocation process (Fig 5.2).

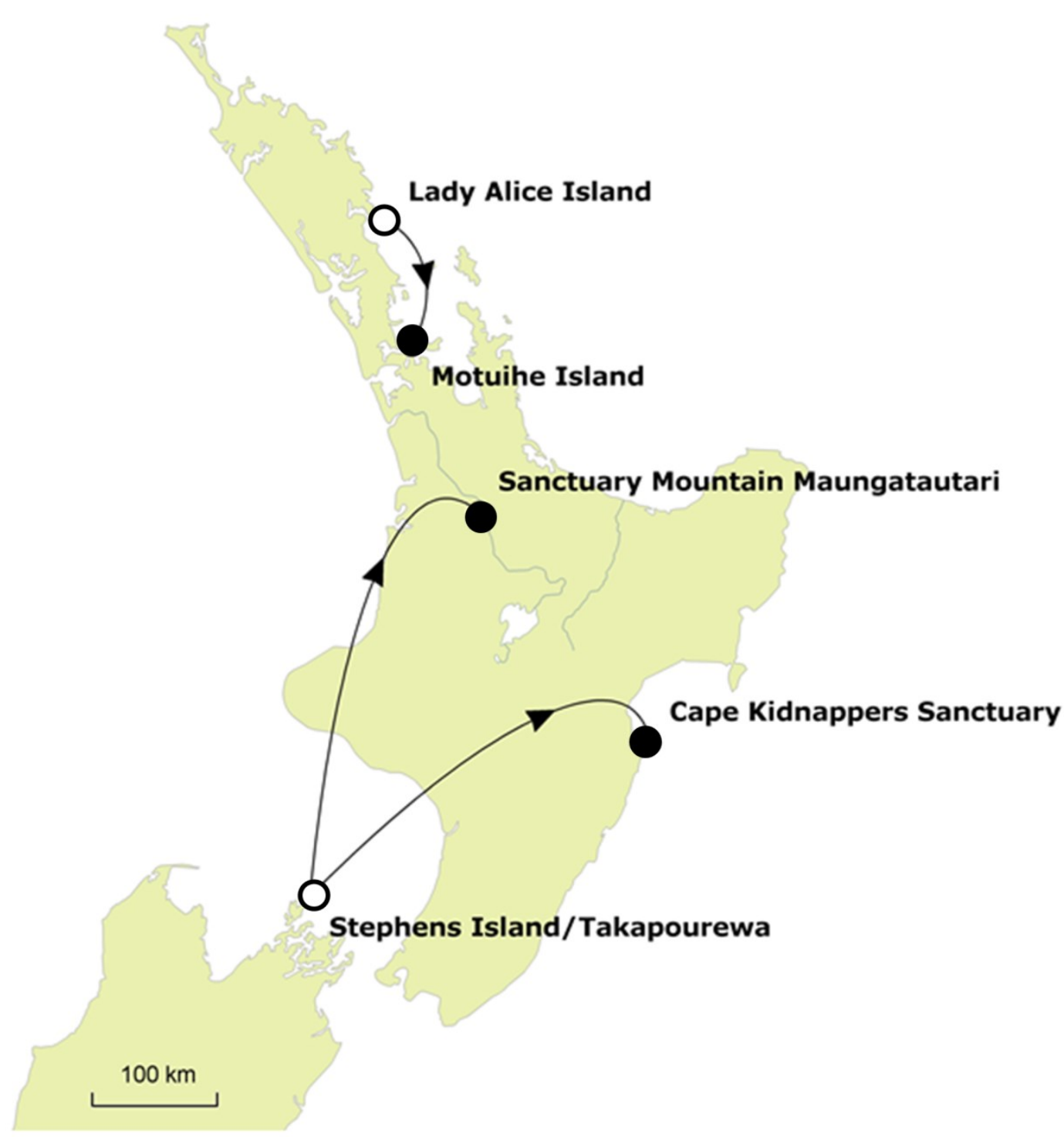

Figure 5.1: Populations involved in short- and long-term monitoring of physiological data (corticosterone) throughout a conservation translocation programme in New Zealand. Source populations (white dots) and translocated populations (black dots) are shown. 


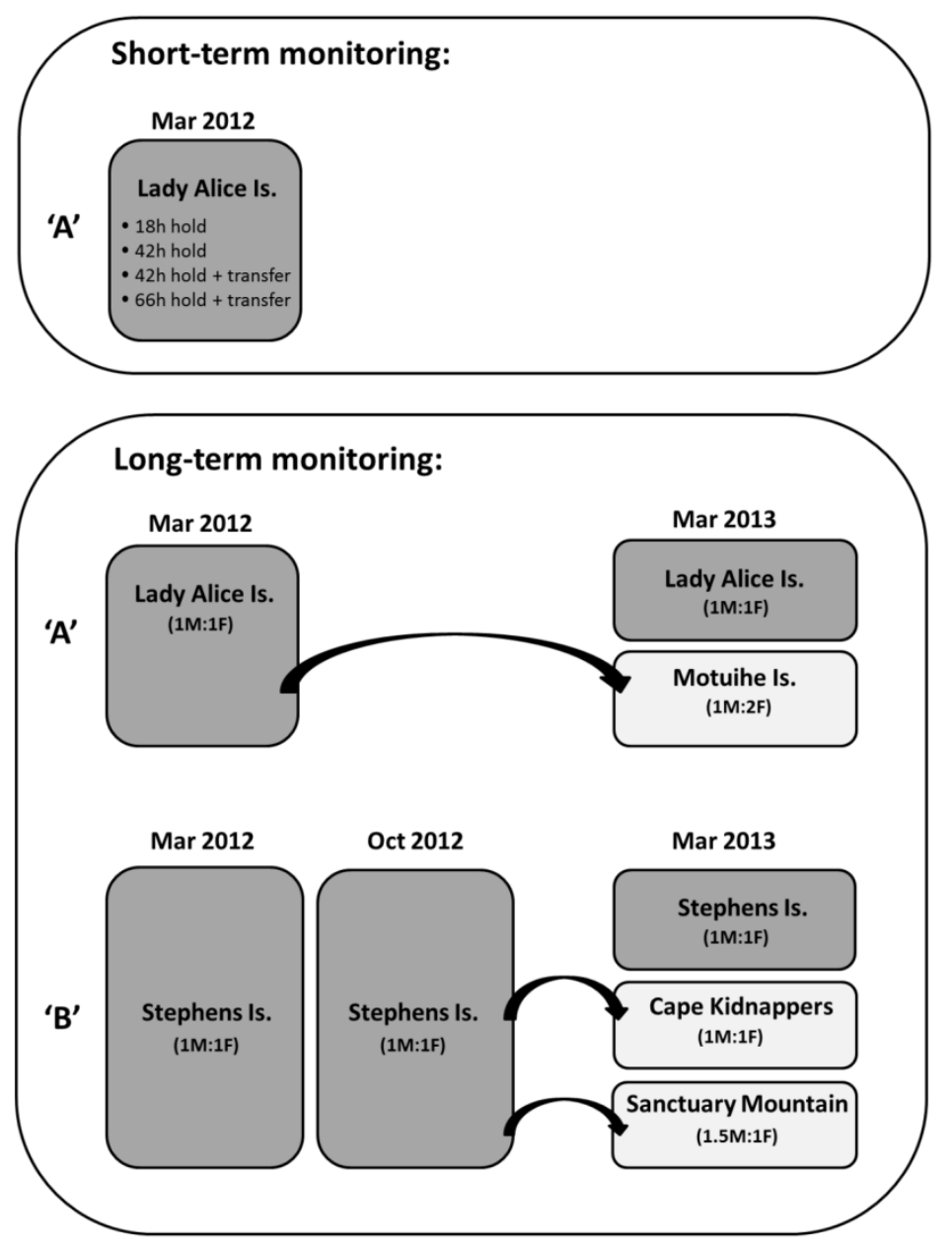

Figure 5.2: Schematic of short-term (upper panel) and long-term (lower panel) monitoring during the translocations to Motuihe Island from Lady Alice Island in March 2012 (Translocation ' $A$ ') and to Cape Kidnappers and Sanctuary Mountain Maungatautari from Stephens Island in October 2012 (Translocation 'B'). Short-term monitoring was carried out during Translocation ' $A$ ' only. Comparisons for long-term monitoring were made between source (dark gray) and translocated (light gray) populations. Sex-ratio (M:F) for each population is shown.

In translocation 'A' (March 2012) wild adult tuatara were translocated to Motuihe

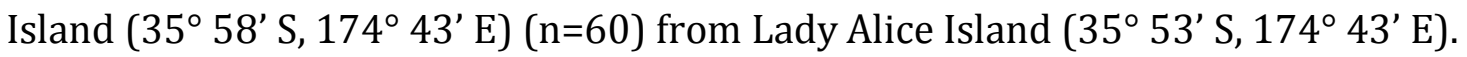
Lady Alice and Motuihe Islands are located in the Northern New Zealand regional climate zone, experiencing sub-tropical warm humid summers and mild winters (NIWA, 2014). In translocation 'B' (October 2012) wild adult tuatara were translocated to five locations from Stephens Island ( $40^{\circ} 40^{\prime} \mathrm{S}, 174^{\circ} 00^{\prime} \mathrm{E}$ ), including Cape Kidnappers Sanctuary (39 64' S, $177^{\circ} 09^{\prime}$ E) $(n=40)$ and Sanctuary Mountain Maungatautari $\left(38^{\circ} 30^{\prime} \mathrm{S}, 175^{\circ} 33^{\prime} \mathrm{E}\right)(n=50)$, which were the two locations 
monitored in this study. Stephens Island is located in the Northern South Island regional climate zone and Cape Kidnappers Sanctuary is located in the Eastern North Island regional climate zone, both experiencing warm dry summers and mild winters (with frost). Sanctuary Mountain Maungatautari is located in the Central North Island regional climate zone, experiencing warm dry summers and cool winters (with frost and fog) (NIWA, 2014). All translocation release sites offered suitable physical habitat for tuatara (with artificial burrows also provided), and social aspects such as M:F sex-ratio (Fig 5.2) and tuatara densities were within normal range.

\subsubsection{Short-term monitoring: The acute corticosterone response at different stages of the initial translocation process}

In translocation 'A' only, I examined the acute CORT response (i.e. CORT secretion above baseline) through all stages of the initial translocation process, during which standard translocation protocols were followed (Cromarty and Alderson, 2013; Towns et al., 1990). In summary, adult tuatara (snout-vent length $\geq 170 \mathrm{~mm}$ ) that were emerged from their underground burrows were captured by hand (between 20:00 $\mathrm{h}$ and 04:00 h) and sex was identified by examining secondary sex characteristics such as head size/shape, body shape, spine shape and crest development (Cree, 2014).

All individuals in the translocation programme were subject to a capture-restraint "hold" (which involved capture of individuals and initial holding (between 40 - $60 \mathrm{~h}$ ) in cloth capture bags), processing (which involved handling, weighing, measuring and implantation of a passive integrated transponder (PIT) tag), and transfer to release site (which involved holding (between $6-10 \mathrm{~h}$ ) in perforated cardboard postal tubes $(10 \mathrm{~cm}$ x $50 \mathrm{~cm})$, movement by foot to the helicopter pick-up site, a 30 min helicopter flight, unloading, and a 30-min handing-over ceremony upon arrival at Motuihe Island). To determine the acute CORT response at different stages of the translocation program, I collected baseline CORT samples (following the blood sampling protocol described in section 5.3.4) from tuatara at capture $(0 \mathrm{~h})(n=54)$ and collected a second sample after either (a) an $18 \mathrm{~h}$ hold $(n=15)$, (b) a $42 \mathrm{~h}$ hold $(n=14)$, (c) a $42 \mathrm{~h}$ hold + process + transfer $(n=11)$, or (d) a $66 \mathrm{~h}$ hold + process + 
transfer $(n=14)$. Tuatara do not show significant daily variation in baseline CORT (Tyrrell and Cree, 1998); therefore, time of day at sampling is unlikely to contribute to variation in CORT secretion in this study.

\subsubsection{Long-term monitoring: Dynamics of corticosterone secretion post- translocation}

Figure 5.2 presents a schematic of samples obtained during translocations ' $\mathrm{A}$ ' and ' $\mathrm{B}$ ' and displays sex-ratios (M:F) for each population. For all samples, adult tuatara (both sexes) were captured at night by hand (between 20:00 h and 04:00 h). Upon capture, a baseline CORT sample was obtained, and after $3 \mathrm{~h}$ capture-restraint in a cloth bag, a second sample was obtained to determine the CORT response. In translocation 'A', I collected samples from the source population (Lady Alice - LA) prior to translocation (March 2012) and from the source (LA) and translocated (Motuihe - Mot) populations at 12 months post-translocation (March 2013). In translocation 'B', I collected samples from the source population (Stephens - ST) prior to translocation (October 2012) and from the source (ST) and translocated (Cape Kidnappers - CK; Sanctuary Mountain Maungatautari - MT) populations at 6 months post-translocation (March 2013). As significant seasonal variation in CORT secretion has been observed between the breeding (March) and non-breeding (October) seasons in tuatara (Chapter Four), I also analysed samples from the ST source population (obtained in a previous study, March 2012, Chapter Four) for annual comparison. To determine if release-site (within a translocated population) had a significant effect on CORT secretion, I collected post-translocation samples from two separate release-site locations on Mot (Site\#1 = Orchards Bush, Site\#2 = Von Luckner's Bush) and at MT (Site\#1= Tuatarium, Site\#2 = Northern Enclosure).

\subsubsection{Sampling protocol}

To determine baseline CORT concentrations, a blood sample (up to $1 \mathrm{ml}$ ) was collected within 10 min of capture from the base of the tail with a heparinized 23gauge needle and $1 \mathrm{ml}$ syringe. After baseline samples were taken, individuals underwent capture-restraint in a cloth capture bag and/or postal tube $(3 \mathrm{~h}-66 \mathrm{~h}$ depending on study), whereupon a second blood sample (up to $1 \mathrm{ml}$ ) was taken to 
determine the CORT response. Internal body temperature $\left(T_{\mathrm{b}}\right)$ was recorded with a cloacal thermocouple (Fluke® Multimeter, model: 179 , specified accuracy $\pm 0.1^{\circ} \mathrm{C}$, USA) prior to taking blood samples from each individual (both baseline and CORT response). After CORT response samples were obtained, individual mass (g) was determined (to the nearest $\pm 5 \mathrm{~g}$ ) with a $1000 \mathrm{~g}$ spring scale (Pesola AG, Switzerland) and snout-vent length $(\mathrm{mm})$, tail length $(\mathrm{mm})$, and tail regeneration length (mm) were measured with a ruler. Body condition scores were generated for each individual as standardized residuals from a regression of log Tail-Corrected Mass (TCM) (Newman et al., 1994) and log SVL (Schulte-Hostedde et al., 2005). Body condition scores were generated separately for each source population (ST and LA) and sex.

Depending on field conditions (i.e. electricity available or not), blood samples were separated either by centrifuge ( $5 \mathrm{~min}$ at $2000 \mathrm{rpm}$ ) or under normal gravity for 6 to $8 \mathrm{~h}$ at $4^{\circ} \mathrm{C}$ (Reimers et al., 1983; Sheriff et al., 2011). Plasma was transferred into cryogenic vials with a micropipette, stored in a cryogenic dry shipper (Thermo ScientificTM, Arctic ExpressTM Dual 10) or in a freezer at $-20^{\circ} \mathrm{C}$ until return to the laboratory, and then stored at $-80^{\circ} \mathrm{C}$ until assayed. Corticosterone was analysed with commercial enzyme immunoassay kits (Cayman Chemical Co., Ann Arbor, MI) using a previously described method validated for tuatara (Chapter Two).

Briefly, CORT was extracted from plasma samples with redistilled dichloromethane and each sample was assayed in duplicate. For each extraction, a subset of tritiated CORT samples were analysed to measure extraction recovery. Mean extraction recovery was $106 \% \pm 8 \%$ s.d. with an overall CV of $7 \%$. Intra-assay and inter-assay CVs were $9.9 \%$ and $14.2 \%$, respectively.

\subsubsection{Statistical analyses}

Data analyses were carried out using R v3.2.0 statistical software (R Development Core 2013) and Prism 6 (Graphpad Software Inc.). All data were checked for assumptions of normality and were transformed if necessary. Linear mixed effects regression (LMER) models were fitted using the 'Ime4' package (Bates, 2013) in R to analyse 1 ) the acute CORT response during the initial translocation process and 2) 
long-term dynamics of CORT secretion in translocated populations. Models were constructed through forward/backward stepwise regression procedures (Field et al., 2012). In all LMER models, a random effect of tuatara ID was included to account for repeat sampling of individuals. The 'ImerTest' package (Kuznetsova, 2013) was used to compute $P$-values for coefficients in final models and significance was assumed at $P<0.05$.

Sex $(M, F)$ and linear covariates of body temperature $\left(T_{\mathrm{b}}\right)$ and body condition score (residuals from $\log$ Mass vs. $\log$ SVL) were not significant predictors of CORT secretion in this study (LMER, $P>0.05$ ), and therefore were not included in final models. Furthermore, the location of release site (within translocated populations) did not have a significant effect on CORT secretion in either translocation study A $(P=0.775)$ or B $(P=0.656)$, therefore; individuals from separate release sites within translocated populations were pooled for further analyses.

In analysis 1 (short-term monitoring), log transformed CORT was the response variable and sample (baseline, $18 \mathrm{~h}$ hold, $42 \mathrm{~h}$ hold, $42 \mathrm{~h}$ hold + processing + transfer, $66 \mathrm{~h}$ hold + processing + transfer) was the input variable. In analysis 2 (long-term monitoring), I first examined whether CORT secretion varied by release site within translocated populations, with log transformed CORT the response variable and input variables of hour ( $0 \mathrm{~h}, 3 \mathrm{~h})$, site (\#1, \#2), and an interaction term of hour x site. Models were fitted to data from Motuihe Island and Sanctuary Mountain Maungatautari, as these translocated populations had two separate release locations. Then, I compared CORT secretion between translocated and source (control) populations, with log transformed CORT the response variable and input variables of hour ( 0 h, 3 h), sample (source pre-, source post-, translocated pre-, translocated post-) and an interaction term of hour x sample. Lastly, I compared body condition pre- and post-translocation, with body condition score

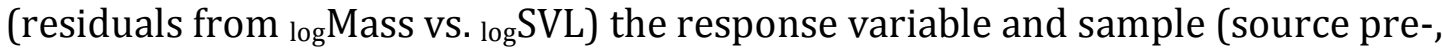
source post-, translocated pre-, translocated post-) the input variable. Models were fitted to data from translocation ' $\mathrm{A}$ ' and 'B'. 


\subsection{Results}

\subsubsection{Short-term monitoring: The acute corticosterone response during different stages of the translocation process}

An acute CORT response (indicated by a significant increase from baseline CORT) was observed in all stages of the translocation process of tuatara from Lady Alice Island to Motuihe Island (Table 5.1, Fig 5.3). The acute CORT response peaked at 18 $\mathrm{h}$ hold and successively decreased (though remaining significantly higher than baseline CORT) at $42 \mathrm{~h}$ hold (LMER, $t=-4.495, P<0.001), 42 \mathrm{~h}$ hold + processing + transfer (LMER, $t=-3.899, P<0.001)$ and $66 \mathrm{~h}$ hold + processing + transfer (LMER, $t=-$ 4.118, $P<0.001$ ) (Fig 5.3).

Contrary to my prediction, cumulative procedures of processing + transfer did not amplify the acute CORT response, as individuals held for $42 \mathrm{~h}$ (without processing + transfer) showed a similar acute CORT response to individuals held for $42 \mathrm{~h}+$ processing + transfer (LMER, $t=0.305, P=0.761$ ) and to individuals held for $66 \mathrm{~h}+$ processing + air-transfer (LMER, $t=0.371, P=0.712)$. Corticosterone concentrations in animals experiencing the latter three treatments were significantly lower than in individuals held for $18 \mathrm{~h}$ only (Table 5.1, Fig 5.3).

Table 5.1: Corticosterone secretion $(\mathrm{ng} / \mathrm{ml}$ ) in tuatara (Sphenodon punctatus) during different stages of the translocation process. Coefficient estimates (positive or negative) are shown and indicate direction of the linear regression from the intercept (baseline CORT $0 \mathrm{~h}$ ). Standard errors (s.e.), $t$-values and $P$-values are shown.

\begin{tabular}{lcccc}
\hline Stages of Translocation Process & estimate & s.e. & t value & $\boldsymbol{P}$ value \\
(Intercept) & 0.400 & 0.038 & 10.39 & $<0.001$ \\
18 h hold & 0.956 & 0.077 & 12.42 & $<0.001$ \\
42 h hold & 0.496 & 0.079 & 6.25 & $<0.001$ \\
42 h hold + process + transfer & 0.530 & 0.088 & 6.01 & $<0.001$ \\
66 h hold + process + transfer & 0.535 & 0.079 & 6.74 & $<0.001$ \\
\hline
\end{tabular}




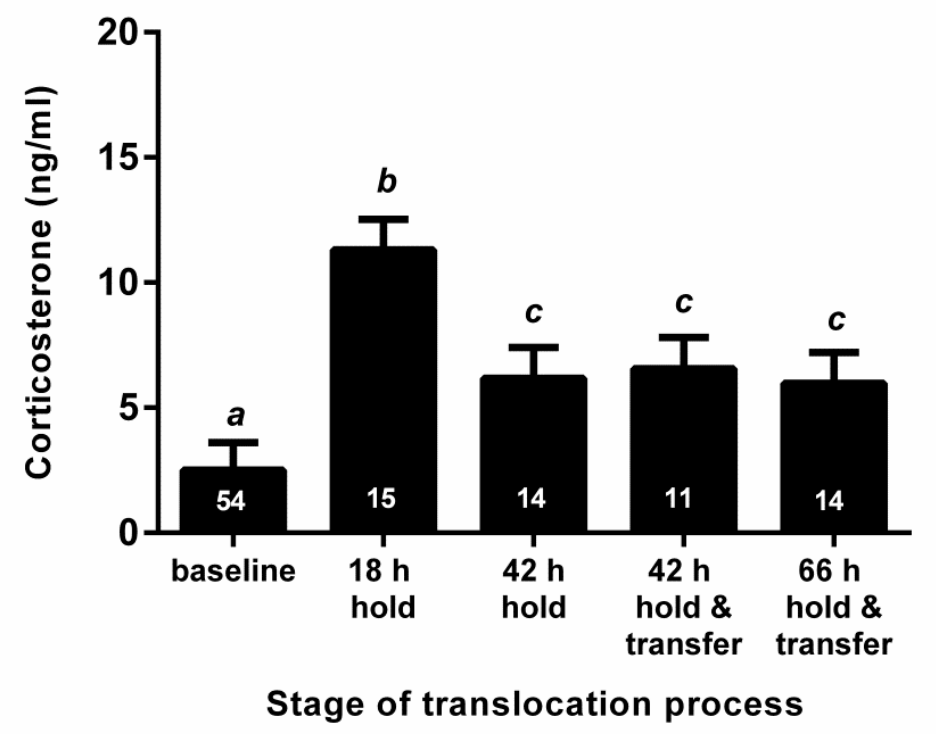

Figure 5.3: Short-term monitoring. The corticosterone response $(\mathrm{ng} / \mathrm{ml}$ ) of tuatara (Sphenodon punctatus) at different stages of the translocation process during translocation ' $A$ ' to Motuihe Island from Lady Alice Island in March 2012. Sample size $(n)$ is indicated by numbers at base of each bar. Bars that share identical letters are not significantly different $(P>0.05)$.

\subsubsection{Long-term monitoring: Dynamics of corticosterone secretion in translocated populations}

In translocation 'A', CORT secretion was similar between translocated (Mot) and source (LA) populations at 12 months post-translocation (Baseline CORT: LMER, $\mathrm{t}=$ 0.163, $\mathrm{P}=0.871$; CORT response: $\mathrm{LMER}, \mathrm{t}=1.136, \mathrm{P}=0.259$, Fig 5.4a). In both populations, baseline CORT was significantly higher at 12 months post-translocation (March 2013), compared to pre-translocation (March 2012); however, the CORT response was similar pre- and post- translocation (Table 5.2a, Fig 5.4a).

In translocation 'B', CORT secretion varied between translocated (CK and MT) and source (ST) populations at six months post-translocation. Baseline CORT was significantly lower in one translocated population (CK) (LMER, $\mathrm{t}=-2.345, \mathrm{P}=0.020)$, but was similar in the other translocated population (MT) (LMER, $\mathrm{t}=-0.925$, $\mathrm{P}=0.356$ ), compared to the source (ST) population (Fig 5.4b). The CORT response was similar in one translocated population (CK) (LMER, $\mathrm{t}=-1.247, \mathrm{P}=0.213$ ), but was significantly higher in the other translocated population (MT) (LMER, $t=1.991$, 
$\mathrm{P}=0.048$ ), compared to the source (ST) population (Fig 5.4b). CORT secretion (both baseline CORT and the CORT response) was similar between the two translocated populations (CK and MT) (Baseline CORT: LMER, $\mathrm{t}=1.210, \mathrm{P}=0.227$; CORT response: LMER, $\mathrm{t}=0.745, \mathrm{P}=0.457$, Fig 5.4b).

CORT secretion in the source (ST) population was similar between both pretranslocation samples (Mar '12 vs Oct'12) (Table 5.2b, Fig 5.4b). In all populations, baseline CORT was significantly higher at six months post-translocation (Mar '13), compared to both pre-translocation samples (Mar '12 and Oct'12) (Table 5.2b, Fig $5.4 \mathrm{~b})$. The CORT response was similar pre- and post-translocation in the two translocated populations (CK and MT), but was significantly lower posttranslocation in the source (ST) population (Table 5.2b, Fig 5.4b).

\subsubsection{Body condition}

In translocation ' $A$ ', body condition was similar between translocated (Mot) and source (LA) populations at 12 months post-translocation (LMER, $t=1.342, P=0.183$, Fig 5.5a). In both populations, body condition was significantly lower at 12 months post-translocation (Mar '13), compared to pre-translocation (Mar '12) (Mot: LMER, $t=-4.632, P<0.001 ;$ LA: LMER, $t=-7.514, P<0.001$, Fig 5.5a).

In translocation 'B', body condition at six months post-translocation was similar in one translocated population (CK) (LMER, $t=-1.351, P=0.179$ ), but was significantly lower in the other translocated population (MT) (LMER, $t=-3.058, P=0.003$ ), compared to the source (ST) population (Fig 5.5b). Furthermore, body condition in the translocated populations (CK and MT) varied as body condition was significantly lower in the (MT) population, compared to the (CK) population (LMER, $t=-4.022$, $P<0.001$, Fig 5.5b). Body condition was similar post-translocation (Mar '13), compared to pre-translocation (Mar '12 and Oct '12), in the translocated (CK) and source (ST) populations, but was significantly lower in the translocated (MT) population (CK: LMER, $t=1.261, P=0.210$; MT: LMER, $t=-3.083, P=0.003$; ST: LMER, $t=-0.077, P=0.938$, Fig 5.5 
a) Translocation A:
LA to Motuine

Baseline CORT

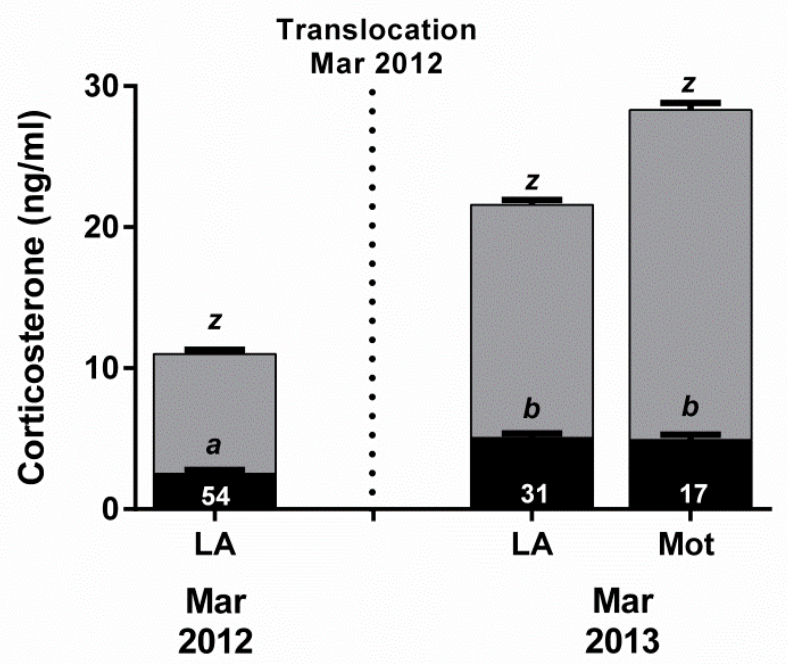

b) Translocation B:

Stephens to CK \& MT

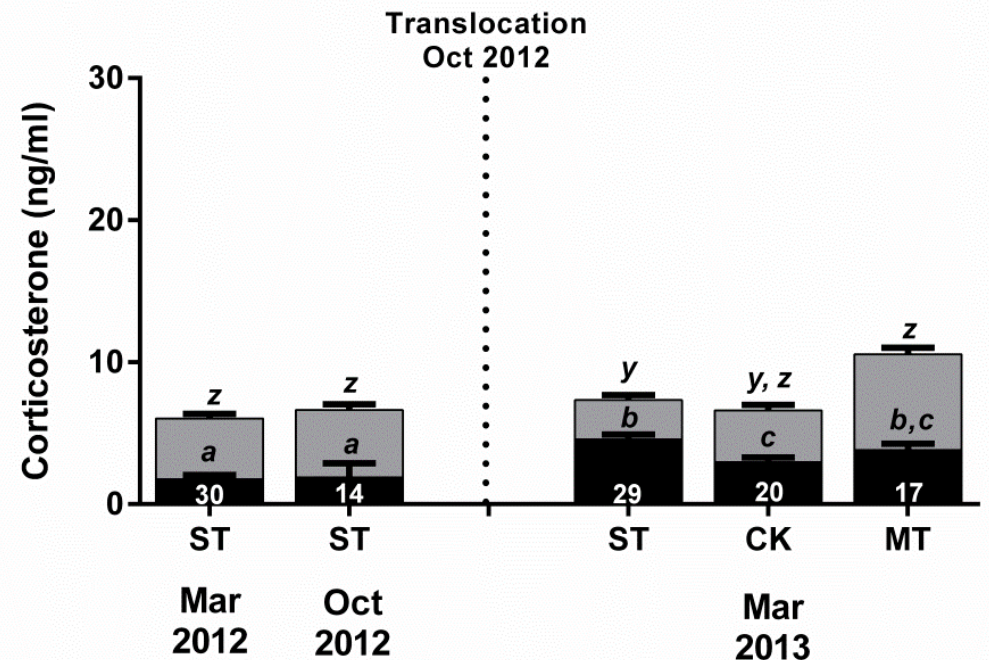

Figure 5.4: Dynamics of corticosterone (CORT) secretion (mean \pm standard error) in populations of tuatara (Sphenodon punctatus) translocated to a) Motuihe Island (Mot) from Lady Alice Island (LA) and b) Cape Kidnappers Sanctuary (CK) and Sanctuary Mountain Maungatautari (MT) from Stephens Island (ST). Sample size $(n)$ is indicated by numbers at the base of each bar and represents a paired sample of baseline CORT (black bars) and the CORT response $(3 \mathrm{~h}-0 \mathrm{~h})$ (gray bars) taken from all individuals. Bars that share identical letters are not significantly different $(P>0.05)$. 
a) Translocation A:

\section{LA to Motuihe}

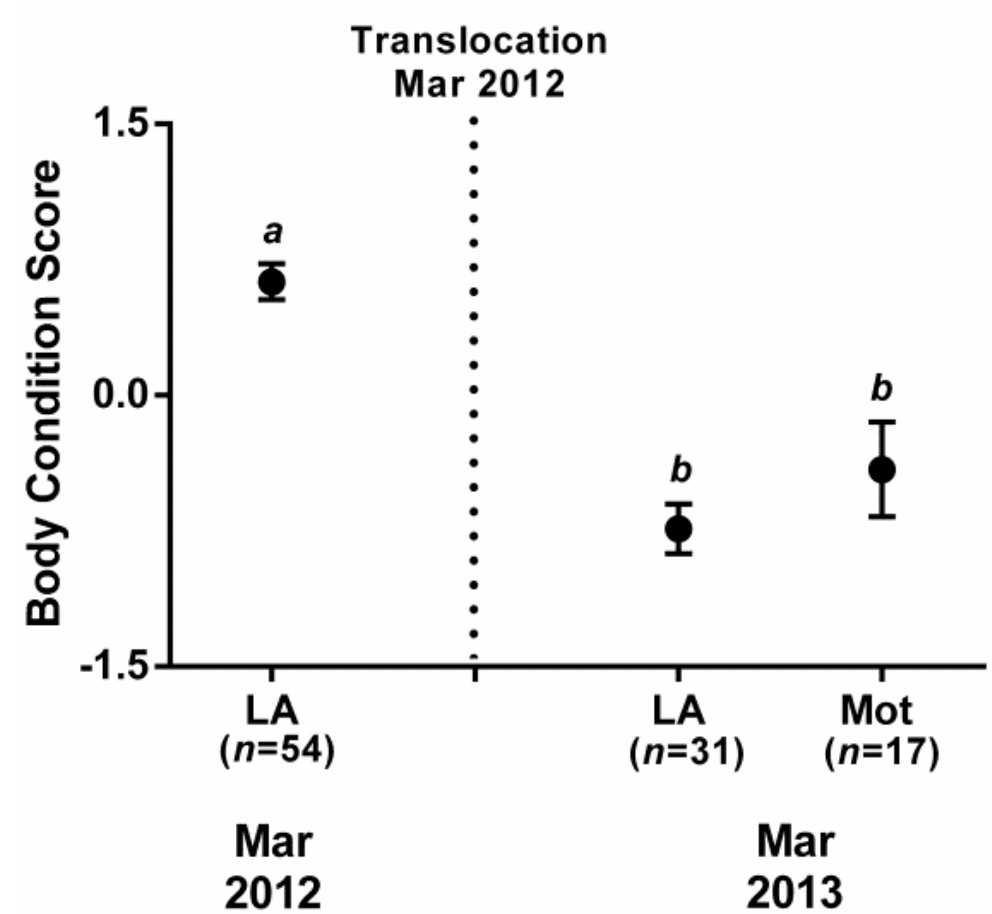

b) Translocation B:

Stephens to CK \& MT

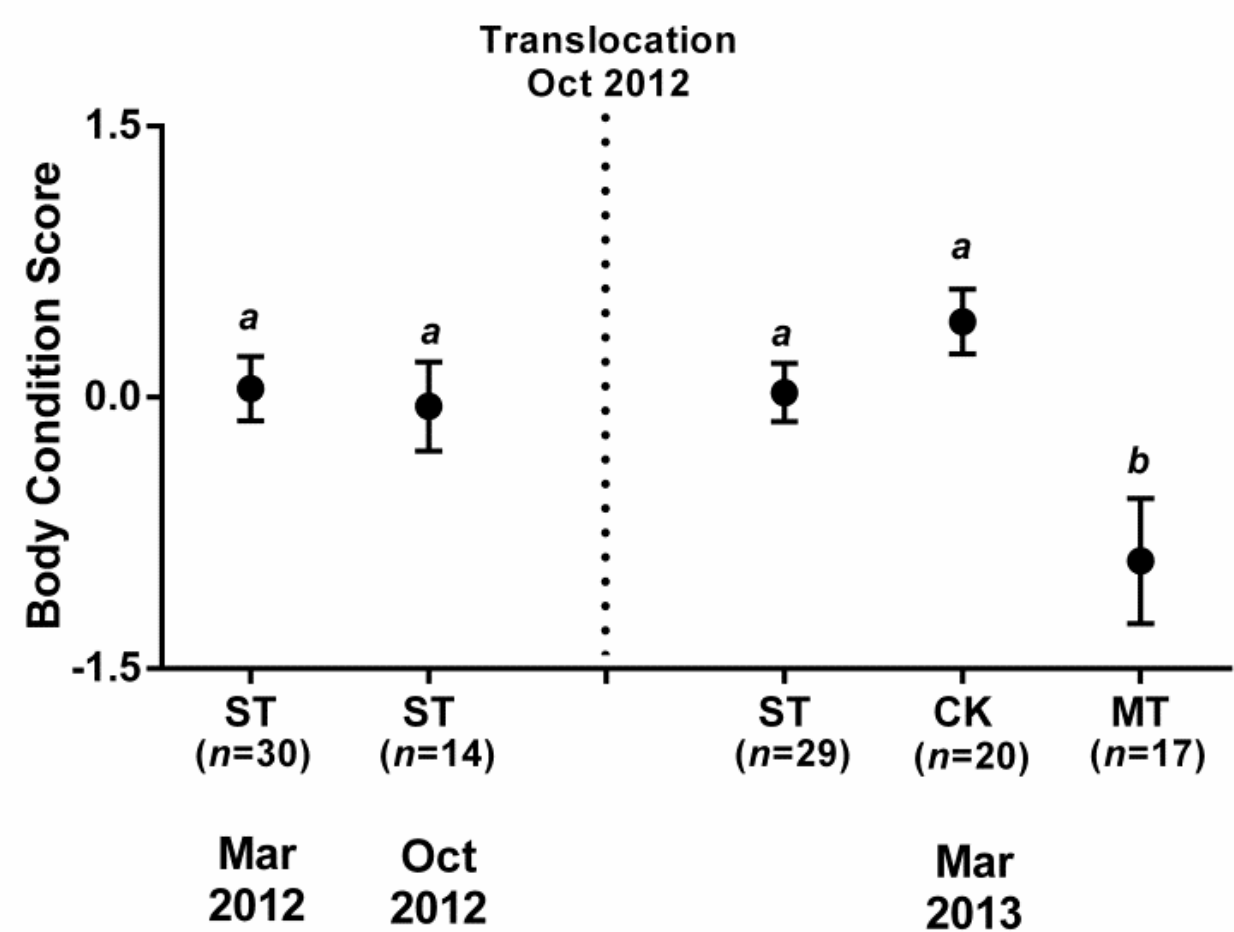

Figure 5.5: Body condition scores (residuals from $\log _{\log }$ Mass vs $\log _{\mathrm{l}} \mathrm{SVL}$ ) in populations of tuatara (Sphenodon punctatus) translocated to a) Motuihe Island (Mot) from Lady Alice Island (LA) and to b) Cape Kidnappers Sanctuary (CK) and Sanctuary Mountain Maungatautari (MT) from Stephens Island (ST). Data points (mean \pm standard error) that share identical letters are not significantly different $(P>0.05)$. 
Table 5.2: Results from linear mixed effects regression (LMER) models examining dynamics of CORT secretion ( $\mathrm{ng} / \mathrm{ml}$ ) pre- and post- translocation to A) Motuihe Island from Lady Alice Island (source population) and B) Cape Kidnappers and Sanctuary Mountain Maungatautari from Stephens Island (source population). Coefficient estimates (positive or negative) are shown and indicate direction of the linear regression from the intercept (baseline CORT $0 \mathrm{~h}$ ). Standard error (s.e.), $t$-values and $P$-values are shown.

Long-term CORT dynamics posttranslocation

Est.

s.e. $t$ value $P$ value

a) Translocation ' $A$ '

Lady Alice (LA) to Motuihe

$\begin{array}{lcccc}\text { (Intercept) } & 0.401 & 0.04 & 9.97 & <0.001 \\ \text { hour } & 0.641 & 0.05 & 12.71 & <0.001^{* * *} \\ \text { post-translocation (LA) } & 0.306 & 0.06 & 4.60 & <0.001^{* * *} \\ \text { post-translocation (Motuihe) } & 0.291 & 0.08 & 3.55 & <0.001^{* * *} \\ \text { hour x post-translocation (LA) } & -0.013 & 0.08 & -0.15 & 0.875 \\ \text { hour x post-translocation (Motuihe) } & 0.115 & 0.10 & 1.10 & 0.271\end{array}$

b) Translocation ' $B$ '

Stephens (ST) to Cape Kidnappers

(CK) and Sanctuary Mountain (MT)

$\begin{array}{lcccc}\text { (Intercept) } & 0.244 & 0.05 & 4.89 & <0.001 \\ \text { hour } & 0.536 & 0.07 & 7.58 & <0.001^{* * *} \\ \text { pre-translocation (Oct ST) } & 0.032 & 0.08 & 0.36 & 0.715 \\ \text { post-translocation (Mar ST) } & 0.414 & 0.07 & 5.81 & <0.001^{* * *} \\ \text { post-translocation (Mar CK) } & 0.227 & 0.07 & 2.88 & 0.004^{* *} \\ \text { post-translocation (Mar MT) } & 0.337 & 0.08 & 4.05 & <0.001^{* * *} \\ \text { hour x pre-translocation (Oct ST) } & 0.008 & 0.12 & 0.06 & 0.945 \\ \text { hour x post-translocation (Mar ST) } & -0.329 & 0.10 & -3.26 & 0.001^{* *} \\ \text { hour x post-translocation (Mar CK) } & -0.188 & 0.11 & -1.68 & 0.093 \\ \text { hour x post-translocation (Mar MT) } & -0.093 & 0.11 & -0.79 & 0.427\end{array}$




\subsection{Discussion}

Here, for the first time, I examined CORT secretion throughout the entire translocation process in a rare reptile (the tuatara, Sphenodon punctatus). I found that 1) plasma CORT concentrations remain elevated throughout the initial translocation process (short-term monitoring between 18 and $66 \mathrm{~h}$ ), but are not amplified by cumulative stressors and 2) the long-term dynamics of CORT secretion are similar in translocated and source populations. Taken together, my results show that tuatara are generally resilient to cumulative acute stressors and to chronic translocation stress.

\subsubsection{Cumulative stressors during translocation do not affect the acute CORT response in tuatara}

To my knowledge, this is the first study to quantify the effect of cumulative stressors (routinely experienced in a translocation) on the acute CORT response in a reptile. I expected to see an effect of additive stressors on the acute CORT response, but this was not the case. The CORT response peaked at $18 \mathrm{~h}$ of holding/captivity-restraint and additional processing procedures of measurements/microchip insertion and air-transfer did not further increase CORT secretion, suggesting resistance to cumulative stressors in this species. Some species show diel variation of CORT secretion (Breuner et al., 1999; Jones and Bell, 2004) which can confound interpretation of results if samples are not taken at $24 \mathrm{~h}$ intervals; however, no evidence of a diel cycle has been found in tuatara (Tyrrell and Cree, 1998). Nevertheless, a significant CORT response was observed throughout all stages of the translocation process, and at no point returned to baseline levels. This observation is consistent with results from my previous study examining the acute CORT response to capture-restraint in tuatara, in which a return to baseline CORT concentrations was not observed over $24 \mathrm{~h}$ (Chapter Two). Therefore, I recommend that animal disturbance, holding time, transport duration, and post-translocation disturbance be minimised in tuatara to mitigate potentially harmful effects of sustained CORT secretion in individuals directly following translocation. 
In the present study, I did not examine patterns of CORT secretion in the immediate weeks following translocation (my first follow-up sampling occurred at 6 months post-translocation). Consequently, I am lacking information on the speed of recovery to baseline CORT secretion levels. Langkilde and Shine (2006) found that CORT secretion in male and female lizards (Eulamprus heatwolei) subject to microchip implantation remained elevated at 14 days (post-treatment), and subsequently increased in response to additional stressors at that time. Similarly, tortoises (Testudo hermanni) that experienced handling plus ground transport had increased baseline CORT at four weeks (post-stressor), compared to a control group that only experienced handling (Fazio et al., 2014). These studies have shown that short-term CORT secretion dynamics are significantly altered by processes experienced during a translocation; therefore, obtaining supplementary information on short-term patterns (within four weeks post-translocation) of CORT secretion in tuatara would shed light on the presence of a sustained CORT response and speed of recovery/negative feedback dynamics following translocation.

\subsubsection{Long-term dynamics of CORT secretion in tuatara are not altered by translocation}

I found that translocation of tuatara did not consistently result in altered CORT secretion relative to controls (source populations) at six or twelve months following translocation (summarised in Table 5.3).

Table 5.3: Summary of long-term dynamics of baseline CORT, the $3 \mathrm{~h}$ CORT response, and body condition at 12 months post-translocation in source (Lady Alice Island, Stephens Island - shown in bold) and translocated (Motuihe Island, Cape Kidnappers, Sanctuary Mountain) populations. Arrows indicate direction of change and asterisks denote level of $P$-value significance.

Population

Lady Alice Is.

Motuihe Is.

Stephens Is.

Cape Kidnappers

Sanctuary Mtn.
Baseline CORT
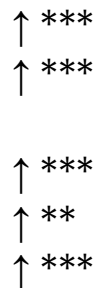

CORT Response

no change no change

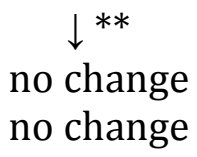

no change
Body Condition

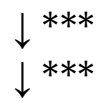

no change

no change 
My results accord with recent studies of translocated reptiles in which CORT secretion was not altered post-translocation. For example, Drake et al. (2012) found that baseline CORT in desert tortoises (Gopherus agassizii) was similar between translocated and control groups at both one- and two- years post-translocation, and both Holding et al. (2014a) and Heiken (2013) found that baseline CORT and the CORT response in translocated northern pacific rattlesnakes (Crotalus oreganus) were not altered post-translocation, compared to controls. In contrast, Gerber et al. (2004) found that baseline CORT in translocated Turks and Caicos iguanas (Cyclura carinata) remained significantly higher than controls at one, five and 12 months following translocation; however, body condition improved and successful reproduction occurred in translocated animals. Although studies are few, my results add to the general reported trend of resilience to translocation and/or translocation stress in reptiles. In contrast, several studies in mammals and birds have reported significant long-term effects of translocation on CORT secretion (Dickens et al., 2009; Franceschini et al., 2008; Gelling et al., 2012; Jachowski et al., 2013; Zidon et al., 2009). However, this observation is not consistent as other studies have reported no long-term effect (Adams et al., 2013; Bosson et al., 2013; Hartup et al., 2005; Ji et al., 2013), suggesting adaptation to new environments (indicated by long-term CORT secretion) is species-specific or context-dependent (e.g. might be due to time of year, weather conditions, or hard- vs soft- release).

Unexpectedly, through long-term monitoring in this study, I observed a significant annual increase in baseline CORT among all source and translocated populations (Fig 5.4, Table 5.3), probably indicating a ubiquitous environmental effect. The CORT response was unaltered in all populations, with the exception of the Stephens Island source population where the CORT response was reduced at 12-months posttranslocation (Table 5.3). Body condition declined in the Lady Alice source population and the Motuihe Island and Cape Kidnappers translocated populations. Moreover, these results highlight the importance of collecting information simultaneously from source populations (as a control), as without this my results of increased baseline CORT in all translocated populations, and reduced body condition in two out of three translocated populations, could have been erroneously interpreted as an indication of chronic stress. 
It is probable that I detected an unplanned/unexpected effect of drought on baseline CORT secretion in tuatara. In 2012 - 2013, New Zealand experienced its worst drought in 40 years, with the North Island affected more severely (Porteous and Mullan, 2013). Lance et al. (2010) observed increased plasma CORT in alligators (Alligator mississippiensis) experiencing a severe drought, and recovery of CORT levels (to within normal limits) was observed after substantial rainfall. Although dehydration stress was not directly measured (by way of CORT secretion), Davis and DeNardo (2009) found that water supplementation in a long-lived desert lizard (the Gila monster, Heloderma suspectum) led to greater hydration, tail-fat reserves, and surface activity. In a recent experimental study, Dupoue et al. (2014) examined CORT secretion in water-deprived snakes (Antaresia childreni) and found that the CORT response, but not baseline CORT, was significantly higher in dehydrated snakes, and the loss of body mass was 2-4 times greater, compared to controls. The authors suggest that baseline CORT in snakes may only respond to a more severe degree of dehydration, and that reduced locomotion (to reduce levels of dehydration) may explain the amplified CORT response in water-deprived snakes (Dupoue et al., 2014).

Reptiles, including tuatara, can moderate water loss through behavioural adaptations such as limiting movement/locomotion and retreating to (or not emerging from) burrows, caves, fallen logs, or undersides of rocks where humidity is higher (Bonnet and Brischoux, 2008; Cree, 2014; Davis and DeNardo, 2009; Wilson et al., 2001). Dunlap (1995) found that lizards (Sceloporus occidentalis) that were more active (compared to less active) during a drought experienced greater changes in physiological measures (e.g., CORT, weight loss, hematocrit, osmolality). Moreover, Dunlap (1995) suggested that individual variation in behavioural responses of reptiles (e.g. remaining active during drought) can lead to biased analysis of stress in natural populations. Burrowing in tuatara reduces water loss by up to three times the rate experienced when emerged (Cree, 2014). Thus, it is possible that the increased baseline CORT observed in my study is influenced by sampling bias (capturing active individuals out of burrows rather than inactive individuals remaining in burrows). 
Contrary to my prediction, a higher CORT response (post-translocation) was not observed in translocated populations experiencing a shift to warmer climates/lower latitudes, specifically the Cape Kidnappers and Sanctuary Mountain translocated populations. In previous studies I observed a higher CORT response in tuatara at higher temperatures (Chapter Three) and at lower latitudes (Chapter Four). The Stephens Island $\left(40^{\circ} 40^{\prime}\right)$ source population showed a reduced CORT response (from pre-translocation to post-translocation), which was not observed in the Cape Kidnappers ( $\left.39^{\circ} 64^{\prime}\right)$ and Sanctuary Mountain (38 30') translocated populations (in which the CORT response was unaltered). Similarly, individuals translocated to Motuihe Island ( $\left.35^{\circ} 58^{\prime}\right)$ from Lady Alice Island ( $\left.35^{\circ} 53^{\prime}\right)$ did not show an altered CORT response. It is possible that the individuals translocated from Stephens Island (to Cape Kidnappers and Sanctuary Mountain) would have shown a lower CORT response (at 12 months) if they remained on Stephens Island, or were translocated to equal or higher latitudes. Examining CORT secretion in tuatara populations translocated to equal/higher latitudes (e.g. to Orokonui Ecosanctuary ( $45^{\circ} 77^{\prime}$ ) from Stephens Island; Cree, 2014), might clarify the effects of latitudinal/climate change on the CORT response.

Although body condition was not significantly correlated with CORT secretion in my study, the sustained body condition in the Cape Kidnappers translocated population, and in the Stephens Island source population, suggests better hydration at these sites in the midst of a drought. Porteous and Mullan (2013) report that New Zealand's South Island (close to where Stephens Island is located) was not affected as severely as the North Island, and better hydration in the Cape Kidnappers population was probably achieved through provision of supplementary water sources (pers. obs.). Reduced body condition has been observed in dehydrated/water-restricted reptiles, including snakes (Dupoue et al., 2014; Lillywhite et al., 2014), lizards (Davis and DeNardo, 2009; Davis and DeNardo, 2010; Dunlap, 1995; Summers and Norman, 1988), alligators (Lance et al., 2010), and turtles (Ray et al., 2004; Ray et al., 2008; van de Merwe et al., 2013). Clearly, information on relationships among water availability/dehydration, body condition, stress, and CORT secretion is lacking, and should be considered in light of imminent climate change. 



\section{Corticosterone secretion in tuatara: Thesis summary and conservation applications}

\subsection{Introduction}

The endocrine system in vertebrates produces glucocorticoid hormones (including corticosterone - CORT) that promote basic life processes, regulate life-history transitions and help individuals cope with intrinsic and extrinsic changes/challenges (i.e. stressors); thereby playing an important fitness role (Boonstra, 2013; Busch and Hayward, 2009; Cockrem, 2013). Here, I examined factors that are associated with CORT secretion in tuatara (Sphenodon punctatus) and measured CORT secretion as a physiological tool to monitor conservation efforts. Using both observational and experimental studies, I identified intrinsic and extrinsic factors that are significantly correlated with CORT secretion (both baseline and the CORT response) and, for the first time, utilised CORT physiology as a monitoring tool in relevant tuatara conservation efforts (i.e. through a Pacific rat (Rattus exulans) eradication and translocation programmes). To date, this thesis delivers the most comprehensive information on CORT physiology in tuatara and provides a foundation for future stress physiology research and baseline data for long-term monitoring of conservation efforts and response to environmental change.

\subsection{Summary of findings}

The main findings from the previous four data chapters are summarised as follows: 


\section{A) Chapter Two: Modulation of corticosterone secretion in tuatara (Sphenodon punctatus): Evidence of a dampened stress response in gravid females}

Sex is not correlated with CORT secretion (either baseline CORT or the CORT response to capture-restraint) in tuatara; however, female reproductive condition is. Gravid females have significantly higher baseline CORT, and a significantly dampened CORT response, compared to non-gravid females (and also males) during the nesting life-history stage. This result supports my hypothesis that gravid females will have a dampened CORT response compared to non-gravid females. The functional significance of elevated baseline CORT secretion in gravid females during nesting is unclear; therefore, experimental studies involving hormone manipulation (in which CORT secretion is increased or decreased) in gravid females would help determine if gravid females are able to mount a significant CORT response (examined in Appendix A; a question re-visited in Chapter Three) and/or if elevated baseline CORT is linked with timing of oviposition (examined in Appendix A).

B) Chapter Three: Body temperature is correlated with the corticosterone stress response in tuatara (Sphenodon punctatus)

The CORT response is positively correlated with internal body temperature $\left(T_{b}\right)$ in male and female (gravid and non-gravid) tuatara, whereas baseline CORT is positively correlated with $T_{\mathrm{b}}$ in gravid females only. Acute increase in mean $T_{\mathrm{b}} 12.0 \pm 0.7^{\circ} \mathrm{C}$ to $21.4^{\circ} \pm 0.4^{\circ} \mathrm{C}$ elicits a significant CORT response in gravid female tuatara (proving that gravid females are in-fact able to mount a further CORT response - a question posed in Chapter Two) and may increase the CORT response in males and non-gravid females. These results support my hypothesis that an acute increase in $T_{\mathrm{b}}$ increases the CORT response to capture-restraint in tuatara. 


\section{C) Chapter Four: The corticosterone stress response varies among island}

populations of tuatara (Sphenodon punctatus) and is associated with linear ecological attributes

Baseline CORT was similar among tuatara populations during the nonbreeding season and the breeding season (which is contrary to my prediction that baseline CORT varies among populations); however, the CORT response to capture-restraint varied significantly among populations. In general, baseline CORT increased, and the CORT response decreased, from the nonbreeding season to the breeding season. This result supports my hypothesis that baseline CORT is higher during the breeding season, compared to the non-breeding season.

Habitat factors of latitude, tuatara density and seabird abundance (PC1 axis) and 2) demogenetic factors of sex ratio and genetic diversity (PC2 axis) are significantly correlated with the CORT response, but not baseline CORT. The correlation with habitat factors provides support for my hypothesis that the CORT response is higher in northern populations that experience a milder climate and the past/recent presence of Pacific rats (Rattus exulans).

Testosterone (T) secretion was not directly associated with CORT secretion; however, inter-population variation in $\mathrm{T}$ was observed in males (but not females) and was positively associated with increased baseline CORT secretion from the non-breeding season to the breeding season, suggesting male reproductive activity may drive the seasonal increase in baseline CORT.

\section{D) Chapter Five: Moving house: Long-term dynamics of corticosterone secretion are unaltered in translocated populations of a rare reptile (the tuatara, Sphenodon punctatus)}

The CORT response to capture-restraint remains elevated during the initial translocation process, but does increase with cumulative stressors. The longterm dynamics of CORT secretion are similar in translocated and source 
populations. Translocated tuatara are generally resistant to cumulative acute stressors and show no hormonal sign of chronic stress. These results do not support my hypothesis that cumulative stressors (experienced during the translocation process) and translocation to a novel location increase CORT secretion. It is hypothesised that drought leads to increased baseline CORT secretion in tuatara.

\subsection{Conservation implications: The value of CORT as a conservation physiology tool in tuatara}

Endocrine techniques have been utilised in ecological studies as a tool to monitor or assess an individual's physiological response to challenges and/or changing environments (Wikelski and Cooke, 2006), and hold great potential to inform and enhance conservation science (Tarszisz et al., 2014). The results of this thesis provide valuable information on intrinsic and extrinsic factors that are associated with CORT secretion in tuatara, which is a necessity when utilising endocrine data as an applied conservation physiology tool.

\subsubsection{Patterns of CORT secretion indicate a physiological response to challenges in tuatara}

Although there is still much to learn about CORT secretion in tuatara (and reptiles in general), this thesis has indicated patterns in baseline CORT and the CORT response in tuatara in response to challenges/stressors:

\subsubsection{Baseline CORT}

In accordance with other vertebrate species (Hamann et al., 2002; Romero, 2002), gravid female tuatara show elevated baseline CORT secretion during the energetically demanding nesting life-history stage (Chapter Two). Similarly, baseline CORT secretion increases (in both males and females) from the non-breeding season to the breeding season (Chapter Four), which suggests an increase in reproductive 
activity (possibly driven by males) (Chapter Four). Baseline CORT likely increased in response to drought conditions and this response was observed among all populations studied here (Chapter Five).

\subsubsection{The CORT response}

Tuatara exhibit a significant CORT response to capture-restraint (Chapter Two Chapter Five; Cree and Tyrrell (2001)), with restraint times ranging from $1.5 \mathrm{~h}$ to 66 h. Throughout all periods of capture-restraint, CORT secretion remained significantly higher than baseline CORT (Chapter Two - Chapter Five).

Cumulative stressors (including handling/measuring, PIT-tagging, extended holding, and helicopter transfer) did not increase the CORT response - which is contrary to what I predicted (Chapter Five), but internal body temperatures ( $\left.T_{\mathrm{b}}\right)$ approaching $21.4^{\circ} \mathrm{C}$ elicit a significant CORT response in gravid females (Chapter Three). A significant CORT response observed in gravid females (in response to increased $T_{\mathrm{b}} /$ capture-restraint) demonstrates that gravid females are indeed able to mount a CORT response during the nesting life-history stage, which under standard capturerestraint conditions is dampened (Chapter Two).

A significant difference in CORT secretion was not observed in response to the recent Pacific rat (Rattus exulans) eradication on Taranga Island (Chapter Four), but my results show that populations that have experienced rats (such as Lady Alice and Taranga Islands) have higher CORT responses compared to populations that have not (such as Stephens and North Brother Island). This result could also be explained as a latitudinal pattern; therefore, further research and/or monitoring of CORT secretion in populations that have experienced Pacific rat introduction/eradication are needed to clarify my results.

The North Brother Island population shows a lower CORT response compared to the other populations studied here, which could be a product of low genetic diversity and/or reproductive activity (Chapter Four). Variation in the magnitude of the CORT response among populations does not necessarily imply that certain environments are more "stressful" than others (Breuner et al., 2008; Breuner et al., 
1999), rather the CORT response is considered to be a measure of an individual's ability to respond to a stressor and individuals in certain populations may simply have a modulated CORT response to adaptively cope with their respective environments. It is likely that variation in the numerous regulatory mechanisms of the HPA axis (stress reactivity, negative feedback, regulation of CORT binding proteins, and adrenal sensitivity) are heritable and linked to variation in fitness (MacDougall-Shackleton et al., 2013).

\subsubsection{CORT secretion is a valuable addition to the conservation 'tool-box' for tuatara}

Results presented in this thesis can be applied toward conservation efforts of tuatara in several ways. The knowledge that female reproductive condition (gravid vs. non-gravid, Chapter Two) and season (breeding vs. non-breeding, Chapter Four) are associated with CORT secretion emphasises the importance of identifying/considering reproductive status/activity. Although testosterone (T) and CORT are not significantly correlated (Chapter Four), seasonal modulation of CORT may inform researchers/managers on the overall reproductive activity level (T) of populations.

Conservation efforts such as population monitoring, research programmes, translocation and captive management programmes routinely employ capturerestraint protocols. These protocols induce a significant CORT response and if carried out for longer time periods (such as in translocations) can constrain normal thermoregulatory behaviours. Knowing that temperature is positively correlated with the CORT response (Chapter Three) informs researchers/managers that caution should be taken when capture-restraint protocols are employed, specifically when longer restraint periods/higher ambient temperatures $\left(>20^{\circ} \mathrm{C}\right)$ occur. Potential solutions to offset a higher CORT response include: ensuring cool holding conditions, providing shaded holding areas during translocation/research programmes, dampening catch bags if required.

Knowing that CORT responses are significantly correlated with ecological attributes experienced by specific populations (Chapter Four) informs managers on the 
population's adaptive response/ability to respond to stressors, which may come in to play when selecting individuals and/or populations for captive breeding or translocation programmes. For example, in a new environment (considered more benign compared to the original environment), a lower CORT response may be advantageous; however, if the new environment is degraded (i.e. wild to captivity) or becomes unpredictable (i.e. warmer temperatures/susceptible to drought/exposure to predators) a greater CORT response may be advantageous for overall fitness (MacDougall-Shackleton et al., 2013). The merit of a lower versus a higher CORT response is an area of research that requires further testing in tuatara (and vertebrates in general).

The lack of apparent chronic stress in translocated populations of tuatara (Chapter Five) provides positive feedback for ongoing conservation translocation efforts in New Zealand. Moreover, the circumstantial evidence of increased baseline CORT in response to drought (Chapter Five) leads to a hypothesis that requires further testing (discussed further in section 6.4) and highlights the potential value of CORT physiology as a tool to quantitatively monitor the physiological response to impending environmental change (i.e. climate change).

\subsection{Recommendations for future research}

This thesis provides the most complete picture of CORT physiology in tuatara to date, but several new research topics (and associated questions) have arisen. A solid foundation has been laid for future research examining more complex details of CORT physiology in tuatara, which will expand on the work presented here.

\section{Topic 1: Relationships between CORT secretion and reproduction}

- What is the functional significance of elevated baseline CORT and/or a dampened CORT response in gravid females during nesting? 
If there is a relation between baseline CORT secretion in gravid female tuatara and timing of oviposition, then manipulation of CORT concentrations (increased or decreased CORT) will induce or delay oviposition. Appendix A presents results from the first hormone manipulation studies investigating modulation of CORT secretion in tuatara (prompted by results obtained in Chapter Two). Unexpectedly, hormone treatments (ACTH to increase CORT secretion; metyrapone to inhibit CORT secretion) did not significantly influence the CORT response to capture-restraint in gravid and non-gravid females compared with saline controls, though the trend of the CORT response for both treatments (in relation to saline controls) was as predicted, and oviposition patterns are suggestive of a functional role for CORT in timing of oviposition (Appendix A). My preliminary results from this experiment can serve as baseline information for future hormone manipulation studies in tuatara, but further studies will be necessary to finetune dosage information. It would be useful to carry out a dose-response study for ACTH and metyrapone administration with several response sampling times in order to determine suitable dosages and durations to observe significant effects (Appendix A).

- Does CORT secretion in gravid females vary based on nesting experience (higher/lower for first-time nesters?). Does density of females on nesting grounds/in captivity influence baseline CORT and/or the CORT response?

It would be useful to determine the influence of nesting experience and density on patterns of CORT secretion in gravid females, as this may explain individual-/population-level variation observed among gravid females (Jessop et al., 1999b; Riechert et al., 2012).

- Do seasonal patterns of CORT reflect levels of reproductive activity?

If reproductive activity is related to seasonal modulation of baseline CORT in tuatara, then populations with low levels of reproductive activity (as indicated by testosterone (T) in plasma samples) will lack seasonal change 
(i.e. increased baseline CORT secretion from the non-breeding season to the breeding season) in baseline CORT. It would be useful to determine levels of testosterone (T) in plasma samples from Taranga to see if patterns are similar to those observed on North Brother Island (Chapter Four). Low concentrations of $\mathrm{T}$ on Taranga would suggest low levels of reproductive activity (for both males and females), which may be a product of Pacific rat presence and/or aged individuals on Taranga (Chapter Four).

Examining the above questions would inform future studies investigating whether and/or how CORT plays a role in oviposition/nesting behaviour and reproductive activity in tuatara, and may inform captive management programmes and island restoration programmes where groups of tuatara fail to reproduce.

\section{Topic 2: Exploring relationships between CORT and fitness measures}

Future research addressing the following questions will provide understanding and insight towards the effect (if any) of CORT secretion on fitness-related performance measures in tuatara.

- What are the fitness implications (if any) of elevated baseline CORT and/or a dampened CORT response in gravid females? Is there evidence of maternal effects: Is maternal CORT transferred to egg yolk, and if so what are the patterns? Does maternal CORT influence hatchling fitness (i.e. size, growth rate, locomotor performance)?

- What are the fitness implications of increased CORT secretion (as was observed in a drought year - Chapter Five)? What are the relationships between CORT secretion and immune function (i.e. wound healing)? Is there a threshold where body condition influences CORT secretion, as has been seen in marine iguanas (Romero and Wikelski, 2001)?

- What is the relationship between individual genetic diversity and the CORT response? North Brother individuals with less genetic diversity may not respond 
as well to stressful environments as those with higher genetic diversity, as has been seen in mammals (Larson et al., 2009; Sarrieau et al., 1998) and invertebrates (Freitak et al., 2014; Reed et al., 2003).

- What factors influence other aspects of the HPA-axis (such as negative feedback efficiency) and what is the relationship (if any) with fitness-related performance measures?

Variation in negative feedback efficiency (which modulates CORT secretion Chapter One, Fig 1.1) determines the magnitude and duration of CORT secretion (both baseline CORT and the CORT response to capture-restraint). For example, longer (i.e. older) red-sided garter snakes (Thamnophis sirtalis parietalis) (Moore et al., 2000a) and degus (Octodon degus) in lower quality habitat (Bauer et al., 2013) both exhibit reduced negative feedback efficiency of CORT secretion.

It would be useful to test negative feedback efficiency in tuatara, by way of a dexamethasone challenge (Romero and Wikelski, 2006), in individuals of a known age and/or in contrasting environmental situations (both in natural conditions and in a controlled laboratory setting). Results from such experiments would clarify whether age (suggested of Taranga individuals, Chapter Four) or resource competition/habitat quality (suggested of North Brother individuals, Chapter Four) explains inter-population variation in CORT secretion by way of a reduced negative feedback mechanism.

\section{Topic 3: Non-invasive measures of CORT in tuatara}

Obtaining blood samples from wild and captive individuals is considered invasive (Sheriff et al., 2011). Several non-invasive measures have been developed to assess/determine CORT in other taxa. Examples include: urinary samples in amphibians (Kindermann et al., 2012; Narayan et al., 2011b), faecal samples in elephants (Jachowski et al., 2013) and horses (Ji et al., 2013), skin sheds in snakes 
(Berkvens et al., 2013), claw trimmings in turtles (Baxter-Gilbert et al., 2014), hair samples in bears (Bechshøft et al., 2012), and feather samples in birds (Bortolotti et al., 2008).

Development and validation of non-invasive measures of CORT secretion for tuatara (for which blood collection training/equipment is not required) would allow for collection of opportunistic samples by management/conservation staff in captivity and in the wild. In captive-breeding facilities/captive collections, samples could be collected from individuals to monitor baseline CORT non-invasively, and analysed for relationships with other measures of fitness (size, growth rate, locomotor performance, wound healing). Development of non-invasive CORT determination would also allow for analysis of younger/smaller age classes (as blood sample collection is difficult in hatchlings and juveniles due to their small size), which is an area that has to date gone un-studied with respect to patterns of CORT secretion.

\subsection{Summary}

The field of conservation physiology is a relatively new discipline, and in order to reliably to use CORT as a conservation tool, one must know before-hand what factors contribute to CORT secretion to effectively control for covariates and interpret results (Dantzer et al., 2014; Wikelski and Cooke, 2006). Measurements of CORT can provide quantitative information about how environmental challenges or stressors impact individuals and populations. Furthermore, monitoring CORT has potential to be used as an 'early warning system' of possible population decline, and threshold levels of CORT could be set and (if reached) prompt implementation of management plans and conservation programmes (Dantzer et al., 2014).

To date, this thesis delivers the most comprehensive information on stress physiology in tuatara, an iconic protected reptile. Intrinsic and extrinsic factors associated with CORT secretion are identified, patterns of CORT secretion in response to challenges/stressors are observed, and the potential value of measuring CORT secretion as a conservation tool in tuatara is explored. These results provide a foundation for future research that will further advance the comparative 
understanding of stress physiology in reptiles. More importantly, understanding stress physiology may be critical for managing future population viability of tuatara in a changing climate, where increased temperatures, changes in water availability, and habitat alteration will present challenges. 


\section{Appendix A: Hormone manipulation studies in gravid tuatara}

Introduction

Gravid females have significantly higher baseline CORT during the nesting lifehistory stage compared with non-gravid females and males (Chapter Two). Baseline CORT is highest during nest digging and oviposition (Cree and Tyrrell, 2001). Interestingly, a distinct fall ( $\sim 5$-fold) in baseline CORT is observed after oviposition, even in the case of females that continue to guard their nests, which suggests a potential role in the timing of oviposition (Cree and Tyrrell, 2001). Nesting is separated by 6-7 months from mating and ovulation in tuatara (Cree, 1994); therefore, patterns in CORT secretion that are related specifically to nesting/oviposition are much more readily identified than in other reptiles.

In Chapter Two, I tested for (and confirmed), a dampened CORT response in gravid female tuatara during the nesting life-history stage. Here, I further examine the responsiveness of gravid females to adrenocorticotrophic hormone (ACTH) and metyrapone challenges during the nesting life-history stage. The pituitary hormone ACTH stimulates CORT release from the adrenal glands, whereas metyrapone is a CORT-synthesis blocker. Both have been used in several vertebrate species, including reptiles, to manipulate CORT synthesis (Cartledge and Jones, 2007; Dixon et al., 1985; Klukowski, 2011; Thaker et al., 2010; Yang and Wilczynski, 2003). I predicted that CORT secretion would be increased in individual tuatara treated with ACTH and inhibited in individuals treated with metyrapone. A reduced response to ACTH by gravid females compared with non-gravid females would suggest that the ability to secrete CORT is reduced during the nesting life-history stage. In exposing tuatara to these challenges, I also aimed to test whether increased or decreased CORT secretion influences oviposition in gravid females during nesting. 
Material and methods

Wild adult tuatara (Sphenodon punctatus) were captured and sampled on Stephens Island/Takapourewa (40 40' S, $174^{\circ} 00^{\prime} \mathrm{E}$ ) in Cook Strait, New Zealand during the October 2012 nesting season. Emerged individuals were caught by hand at night between 20:00h and 05:00h and were subsequently assigned to an appropriate group based reproductive status (gravid female or non-gravid female). Gravid females were selectively captured at or near a nesting rookery and female reproductive status was inferred through abdominal palpation for shelled eggs.

Studies investigating adrenocortical stress-responsiveness use physiological challenges to confirm that individuals can mount a stress response (Romero, 2002). In this study, gravid female $(n=5)$ and non-gravid females $(n=5)$ were subject to an ACTH challenge to determine if gravid females are capable of mounting a CORT response during nesting. In conjunction, I administered a metyrapone challenge to different gravid $(n=5)$ and non-gravid females $(n=5)$ to test whether increased or decreased CORT secretion influences oviposition. Saline was administered to other gravid ( $n=5)$ and non-gravid $(n=4)$ females as a control treatment.

Capture/sampling occurred over 5 nights during the 2012 nesting season (16-20 October). Reproductive condition of females was inferred by abdominal palpation and baseline blood samples were collected within 10 minutes of capture, as described in Chapter Two. Body mass (g) was measured to calculate hormone dosage specific to mass. Individuals were randomly assigned to a treatment of either ACTH (0.03 IU/ $\mu \mathrm{l} / \mathrm{g}$ body mass; Sigma A6303, fragments 1-39 porcine, SigmaAldrich, NZ), metyrapone $(30 \mu \mathrm{g} / \mu \mathrm{l} / \mathrm{g}$ body mass; 96\% 2-methyl-1,2-di-3-pyridyl-1propanone, Sigma-Aldrich, NZ) or $0.9 \%$ saline ( $1 \mu \mathrm{l} / \mathrm{g}$ of body mass). These dosages were chosen on the basis of comparable dosages in other reptile species determined to be effective in producing CORT responses within normal physiological limits (Cartledge and Jones, 2007; Klukowski, 2011; Preest et al., 2005; Romero and Wikelski, 2006; Scholnick et al., 1997; Yang and Wilczynski, 2003). Hormone solutions were freshly prepared at the field site and were held at $4^{\circ} \mathrm{C}$ until 
administered. Treatments were injected intraperitoneally with a $25 \mathrm{G}$ needle and 1 $\mathrm{ml}$ syringe. Individuals were subsequently subject to $3 \mathrm{~h}$ of capture-restraint stress in individual cloth bags and upon completion were re-bled to determine the effect of capture-restraint, injection and hormone treatment on CORT secretion. CORT concentrations were determined by EIA (as described in Chapter Two).

To test if stimulation (ACTH) or inhibition (metyrapone) of CORT secretion influences oviposition during nesting, I held all individuals in clean cardboard boxes (a standard protocol for induction studies in tuatara (Cree et al., 1991a)) for five days following injection and blood sampling. For the duration of holding, individuals were checked daily for signs of oviposition and daily measurements of body mass (g) and skin temperature $\left({ }^{\circ} \mathrm{C}\right.$ ) of the dorsal body were taken with a $1000 \mathrm{~g}$ spring scale and handheld infrared temperature gun (Hare et al., 2007), respectively, between 14:00h and 16:00h.

Data analyses were carried out using R v3.0.0 statistical software (R Development Core 2008) and Prism 6 (Graphpad Software Inc.). All data were checked for normal distributions and homoscedasticity, and if necessary, were transformed to meet assumptions for parametric statistical tests. Linear mixed effects regression (LMER) models were fitted using the 'Ime4' (Bates, 2013) package in R to investigate the effect of ACTH and metyrapone hormone administration (compared to saline controls) on the CORT response in gravid and non-gravid females after $3 \mathrm{~h}$ of capture-restraint. Log-transformed CORT was the response variable. Predictors were group (gravid female, non-gravid female), time, and treatment as fixed main effects (with interaction terms included for all). Tuatara ID was included as a random effect to account for repeat sampling of individuals for baseline and stressresponse CORT values. The 'languageR' package (Baayen, 2011) was used to compute $P$-values based on Markov-chain Monte Carlo (MCMC) sampling. Significance for all tests was assumed at $p<0.05$. 
Results

Unexpectedly, ACTH and metyrapone treatments did not significantly influence the CORT stress response in gravid and non-gravid females compared with saline controls (Table A.1), though the trend of the CORT response for both treatments (in relation to saline controls) was as predicted (Fig A.1). I did not observe a significant effect of treatment on oviposition. During the 5-day holding period, one female from the ACTH group produced two eggs and one female from the saline group produced one egg, with no females from the metyrapone group producing eggs. Furthermore, a significant CORT response to $3 \mathrm{~h}$ capture-restraint and treatment was observed in non-gravid females (Table A.1b), but not in gravid females (Table A.1).

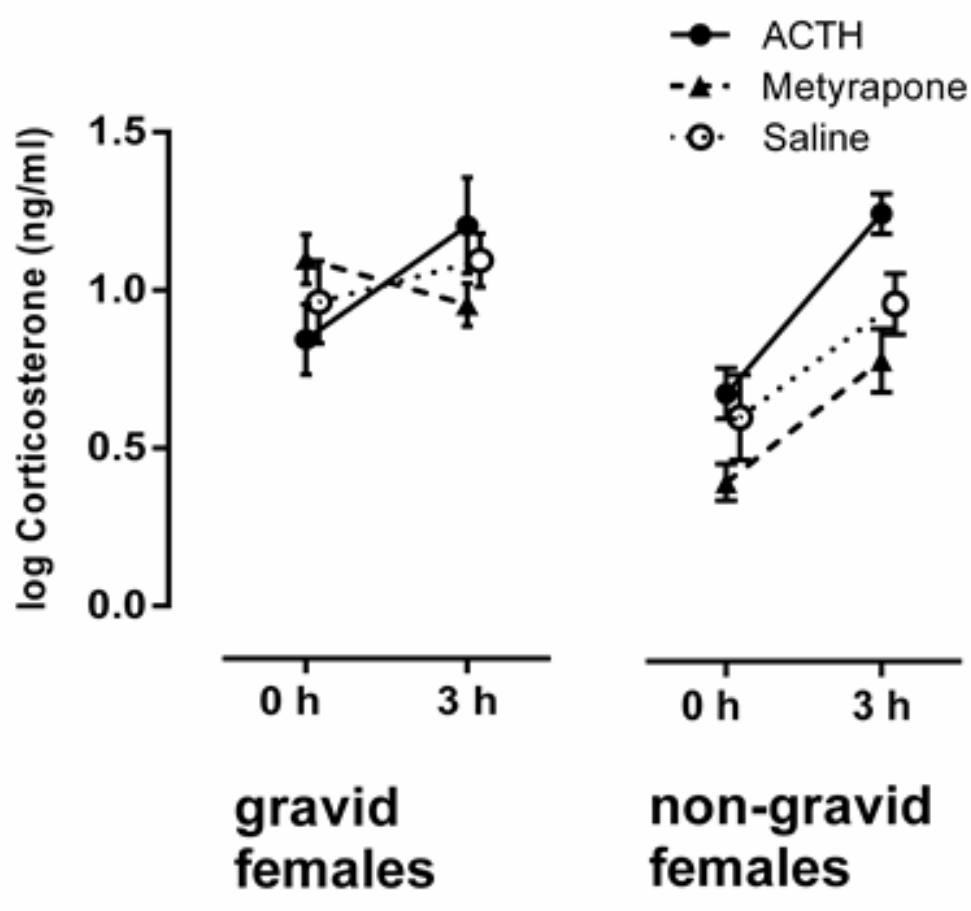

Figure A.1: CORT response to $3 \mathrm{~h}$ capture-restraint following either an ACTH challenge (solid line $\mathrm{w} /$ closed circles), metyrapone challenge (dashed line $\mathrm{w} /$ triangles) or saline control (dotted line $\mathrm{w} /$ open circles) in gravid and non-gravid female tuatara (Sphenodon punctatus). Data points represent mean \pm SE log CORT concentration $(\mathrm{ng} / \mathrm{ml})$ of baseline $(0 \mathrm{~h})$ and stress response $(3 \mathrm{~h})$ samples. No significant difference was found between treatment and saline controls for either group ( $P>0.05$ ). 
Table A.1: Results from linear mixed effects regression models explaining variation in measures of baseline and stress response CORT secretion $(\mathrm{ng} / \mathrm{ml})$ as a function of time $(0-3 \mathrm{~h})$ after explaining variation accounted for by the fixed effects of hormone treatment $(\mathrm{ACTH}$, metyrapone, saline), capture-restraint of $3 \mathrm{~h}$ and the interaction of these effects for a) gravid females and b) non-gravid females.

Fixed effect predictor

\section{c) gravid females}

saline (intercept)

ACTH

metyrapone

capture-restraint time (saline)

capture-restraint time x ACTH

capture-restraint time $\mathrm{x}$

metyrapone

\section{d) non-gravid females}

saline (intercept)

ACTH

metyrapone

capture-restraint time (saline)

capture-restraint time x ACTH

capture-restraint time $\mathrm{x}$

metyrapone
Estimate
0.962

$-0.118$

0.136

0.132

\subsection{8}

0.153

0.153

0.153

0.217

0.217

$-0.276$

0.575

0.073

$-0.197$

0.345

0.201

0.025
0.092

0.124

0.124

0.110

0.148

$0.148 \quad 0.169$
$<0.001$

$-0.769 \quad 0.449$

$0.887 \quad 0.384$

$0.861 \quad 0.398$

$1.051 \quad 0.304$

$-1.273 \quad 0.215$

Coefficient estimates (positive or negative) are shown and indicate direction of the linear regression from the specified intercept. 95\% credible intervals (CI) are shown. P-values based on MCMC sampling are shown and statistically significant results are indicated in bold. A random intercept term for individual was included in the model.

Discussion

In an attempt to elucidate the underlying causes of the dampened CORT response in gravid female tuatara, I administered an ACTH challenge to determine if gravid females are operating at maximal CORT secretion during the nesting life-history stage. I also used ACTH and metyrapone treatments to explore the hypothesis that modulation of CORT secretion influences the timing of oviposition. Unexpectedly, I 
did not observe a significant effect from either treatment compared to saline controls in gravid or non-gravid females. However, I did observe a response to $3 \mathrm{~h}$ capture-restraint in the saline groups that is consistent with my $24 \mathrm{~h}$ capturerestraint results, namely that gravid females had a dampened stress response compared to non-gravid females.

It is surprising that plasma CORT concentrations were unaffected by both ACTH and metyrapone hormone challenges, as studies on other reptile species have seen marked responses from similar doses in shorter time-frames than in this study (Cartledge and Jones, 2007; Klukowski, 2011; Preest et al., 2005; Romero and Wikelski, 2006). A possible explanation could be that the timing of the CORT response sample at $3 \mathrm{~h}$ was either too soon or too late to detect a significant treatment effect. Lack of significant effect from hormonal treatment at certain sample times was observed in related studies; for example, ACTH challenges produced plasma CORT concentrations that were significantly higher than saline controls at $1 \mathrm{~h}$ and $6 \mathrm{~h}$ only in male alligators (Alligator mississippiensis) (Mahmoud et al., 1996) and in New Zealand common geckos at $1 \mathrm{~h}$ only (Preest et al., 2005), but not at any other sample time points tested in either study. Another possibility explaining the absence of a significant treatment effect could be that my calculated hormone dosages may have been too low for my model species. Tuatara are a temperate climate ectotherm (with nocturnal $\mathrm{T}_{b}$ largely tracking ambient temperature), and I planned hormone dosage based on other reptiles that may experience warmer temperatures and therefore may have different rates of hormone metabolism. My preliminary results will be useful as a starting point to inform potential studies investigating mechanisms of CORT modulation in tuatara during nesting. Due to permitting constraints and research timelines, I was unable to carry out a pilot study beforehand. Therefore, it would be beneficial to investigate the dose-dependent response to both ACTH and metyrapone treatment over a timeseries to inform future research and to understand mechanisms and importance of CORT secretion in tuatara when challenged with exogenous hormones.

In the present study, I did not observe an influence of ACTH or metyrapone treatment on oviposition, but as plasma CORT concentrations were not significantly 
affected by treatments in this study, these results do not illuminate my hypothesis. The functional significance of elevated baseline CORT in gravid oviparous reptiles (and the potential relationship with oviposition) has received little attention, even though several oviparous reptile species exhibit elevated concentrations of baseline CORT concentrations directly preceding oviposition (Cree and Tyrrell, 2001; Jessop, 2001; Moore and Jessop, 2003). Female tuatara have a unique egg development/maintenance strategy, retaining their eggs for the longest known period in reptiles (7-9 months) (Cree et al., 1992). Therefore, elevated baseline CORT concentrations during the nesting life-history stage in tuatara cannot be attributed to sexual receptivity or ovulation, as these mating events are greatly separated in time from nesting. A prior study on tuatara shows that baseline CORT concentrations decline shortly after oviposition (Cree and Tyrrell, 2001) , which suggests a possible role in the timing of egg-laying, and other studies suggest hormonal control of ovulation and parturition in viviparous and oviparous reptiles (Jones and Guillette, 1982). For example, it was shown that embryonic production of CORT may trigger parturition in viviparous southern snow skinks (Niveoscincus microlepidotus) (Girling and Jones, 2006) and exogenous elevation of baseline CORT concentrations in gravid eastern three-lined skinks (Bassiana duperreyi) induced 'premature' oviposition (Radder et al., 2008). These findings suggest that upregulating baseline CORT secretion during nesting could function to stimulate oviposition in gravid reptiles.

\section{Conclusions}

I provide results from the first hormone manipulation studies investigating modulation of CORT secretion in tuatara. While the present study provides baseline information for future hormone manipulation studies in tuatara, further studies will be necessary. It would be useful to carry out a dose-response study for ACTH and metyrapone administration with several response sampling times in order to determine suitable dosages and durations to observe significant effects. This would inform future studies investigating whether CORT is being secreted at maximal levels during nesting and if/how it plays a role in oviposition and nesting behaviour in tuatara, the sole extant representative of an ancient order of reptiles. 



\section{References}

Adams N, Claussen DL, Skillings J (1989) Effects of temperature on voluntary locomotion of the eastern box turtle, Terrapene carolina carolina. Copeia: 905-915

Adams NJ, Parker KA, Cockrem JF, Brunton DH, Candy EJ (2013) Inter-island differences in the corticosterone responses of North Island Saddlebacks (Philesturnus rufusater) in New Zealand do not suggest selective effects of translocation. Emu 113: 45-51

Aidam A, Michel CL, Bonnet X (2013) Effect of ambient temperature in neonate Aspic vipers: growth, locomotor performance and defensive behaviors. Journal of Experimental Zoology Part A-Ecological Genetics and Physiology 319A: 310-318

Almasi B, Rettenbacher S, Muller C, Brill S, Wagner H, Jenni L (2012) Maternal corticosterone is transferred into the egg yolk. General and Comparative Endocrinology 178: 139-144

Almasi B, Roulin A, Jenni L (2013) Corticosterone shifts reproductive behaviour towards selfmaintenance in the barn owl and is linked to melanin-based coloration in females. Hormones and Behavior 64: 161-171

Angelier F, Wingfield JC (2013) Importance of the glucocorticoid stress response in a changing world: Theory, hypotheses and perspectives. General and Comparative Endocrinology 190: 118128

Anson JR, Dickman CR, Boonstra R, Jessop TS (2013) Stress triangle: Do introduced predators exert indirect costs on native predators and prey? PLOS ONE 8:

Armstrong DP, Seddon PJ (2008) Directions in reintroduction biology. Trends in Ecology \& Evolution 23: 20-25

Baayen R (2011) languageR: Data sets and functions with "Analyzing Linguistic Data: A practical introduction to statistics" http://cran.r-project.org/web/packages/languageR/

Baird TA, Lovern MB, Shine R (2014) Heightened aggression and winning contests increase corticosterone but decrease testosterone in male Australian water dragons. Hormones and Behavior 66: 393-400

Baker MR, Gobush KS, Vynne CH (2013) Review of factors influencing stress hormones in fish and wildlife. Journal for Nature Conservation 21: 309-318

Barry M, Cockrem JF, Brunton DH (2010) Seasonal variation in plasma corticosterone concentrations in wild and captive adult Duvaucel's geckos (Hoplodactylus duvaucelii) in New Zealand. Australian Journal of Zoology 58: 234-242 
Barwick RE (1982) Observations on active thermoregulation in the tuatara, Sphenodon punctatus (Reptilia: Rhynchocephalia). In Newman DG ed, New Zealand Herpetology, Wellington: New Zealand Wildlife Service, pp 225-236

Bates D (2013) Linear mixed-effects models using S4 classes - R Package 'Ime4' http://Ime4.rforge.r-project.org/

Bauer CM, Skaff NK, Bernard AB, Trevino JM, Ho JM, Romero LM, Ebensperger LA, Hayes LD (2013) Habitat type influences endocrine stress response in the degu (Octodon degus). General and Comparative Endocrinology 186: 136-144

Baxter-Gilbert JH, Riley JL, Mastromonaco GF, Litzgus JD, Lesbarrères D (2014) A novel technique to measure chronic levels of corticosterone in turtles living around a major roadway. Conservation Physiology 2:

Bechsh $\varnothing \mathrm{ft}$ T $\varnothing$, Rigét FF, Sonne C, Letcher RJ, Muir DCG, Novak MA, Henchey E, Meyer JS, Eulaers I, Jaspers VLB et al. (2012) Measuring environmental stress in East Greenland polar bears, 1892-1927 and 1988-2009: What does hair cortisol tell us? Environment International 45: $15-21$

Belliure J, Meylan S, Clobert J (2004) Prenatal and postnatal effects of corticosterone on behavior in juveniles of the common lizard, Lacerta vivipara. J Exp Zool Part A: Comparative Experimental Biology 301A: 401-410

Berger S, Martin LB, Wikelski M, Romero LM, Kalko EKV, Vitousek MN, Rodl T (2005) Corticosterone suppresses immune activity in territorial Galápagos marine iguanas during reproduction. Hormones and Behavior 47: 419-429

Berger S, Wikelski M, Romero LM, Kalko EK, Roedl T (2007) Behavioral and physiological adjustments to new predators in an endemic island species, the Galapagos marine iguana. Hormones and Behavior 52: 653-663

Berkvens CN, Hyatt C, Gilman C, Pearl DL, Barker IK, Mastromonaco GF (2013) Validation of a shed skin corticosterone enzyme immunoassay in the African House Snake (Lamprophis fuliginosus) and its evaluation in the Eastern Massasauga Rattlesnake (Sistrurus catenatus catenatus). General and Comparative Endocrinology 194: 1-9

Berner NJ, Heil LA, Romero LM (2013) Seasonal variation in corticosterone in free-living and captive eastern red-spotted newts Notophthalmus viridescens viridescens. Journal of Herpetology 47: 466-470

Besson AA, Cree A (2010) A cold-adapted reptile becomes a more effective thermoregulator in a thermally challenging environment. Oecologia 163: 571-581

Besson AA, Cree A (2011) Integrating physiology into conservation: an approach to help guide translocations of a rare reptile in a warming environment. Animal Conservation 14: 28-37

Blair TA, Cree A, Skeaff CM (2000) Plasma fatty acids, triacylglycerol and cholesterol of the tuatara (Sphenodon punctatus punctatus) from islands differing in the presence of rats and the abundance of seabirds. Journal of Zoology, London 252: 463-472 
Bliley JM, Woodley SK (2012) The effects of repeated handling and corticosterone treatment on behavior in an amphibian (Ocoee salamander: Desmognathus ocoee). Physiology \& Behavior 105: 1132-1139

Bonier F, Martin PR, Moore IT, Wingfield JC (2009) Do baseline glucocorticoids predict fitness? Trends in Ecology \& Evolution 24: 634-642

Bonnet X, Brischoux F (2008) Thirsty sea snakes forsake refuge during rainfall. Austral Ecology 33: $911-921$

Bonnet X, Fizesan A, Michel CL (2013) Shelter availability, stress level and digestive performance in the Aspic viper. Journal of Experimental Biology 216: 815-822

Boonstra R (2013) The ecology of stress: a marriage of disciplines. Functional Ecology 27: 7-10

Bortolotti GR, Marchant TA, Blas J, German T (2008) Corticosterone in feathers is a long-term, integrated measure of avian stress physiology. Functional Ecology 22: 494-500

Bosson CO, Palme R, Boonstra R (2013) Assessing the impact of live-capture, confinement, and translocation on stress and fate in Eastern gray squirrels. Journal of Mammalogy 94: 1401-1411

Breuner CW, Delehanty B, Boonstra R (2013) Evaluating stress in natural populations of vertebrates: total CORT is not good enough. Functional Ecology 27: 24-36

Breuner CW, Hahn TP (2003) Integrating stress physiology, environmental change, and behavior in free-living sparrows. Hormones and Behavior 43: 115-123

Breuner CW, Patterson SH, Hahn TP (2008) In search of relationships between the acute adrenocortical response and fitness. General and Comparative Endocrinology 157: 288-295

Breuner CW, Wingfield JC, Romero LM (1999) Diel rhythms of basal and stress-induced corticosterone in a wild, seasonal vertebrate, Gambel's white-crowned sparrow. Journal of Experimental Zoology 284: 334-342

Bryan HM, Darimont CT, Paquet PC, Wynne-Edwards KE, Smits JEG (2014) Stress and reproductive hormones reflect inter-specific social and nutritional conditions mediated by resource availability in a bear-salmon system. Conservation Physiology 2: 1-18

Busch DS, Hayward LS (2009) Stress in a conservation context: a discussion of glucocorticoid actions and how levels change with conservation-relevant variables. Biological Conservation 142: 2844-2853

Buxton RT, Currey CA, Lyver POB, Jones CJ (2013) Incidence of plastic fragments among burrow-nesting seabird colonies on offshore islands in northern New Zealand. Marine Pollution Bulletin 74: 420-424

Callard G, Chan SC, Callard I (1975) Temperature effects on ACTH-stimulated adrenocortical secretion and carbohydrate metabolism in the lizard (Dipsosaurus dorsalis). Journal of comparative physiology 99: 271-277

Cartland LK, Grimmond NM (1994) The effect of temperature on the metabolism of juvenile tuatara Sphenodon punctatus. New Zealand Journal of Zoology 21: 373-378 
Cartledge VA, Jones SM (2007) Does adrenal responsiveness vary with sex and reproductive status in Egernia whitii, a viviparous skink? General and Comparative Endocrinology 150: 132139

Cassey P, Ussher G (1999) Estimating abundance of tuatara. Biological Conservation 88: 361-366

Castanet J, Newman DG, Saint Girons H (1988) Skeletochronological data on the growth, age, and population structure of the tuatara, Sphenodon punctatus, on Stephens and Lady Alice Islands, New Zealand. Herpetologica 44: 25-37

Cockrem JF (2013) Individual variation in glucocorticoid stress responses in animals. General and Comparative Endocrinology 181: 45-58

Cockrem JF, Silverin B (2002) Variation within and between birds in corticosterone responses of great tits (Parus major). General and Comparative Endocrinology 125: 197-206

Corkery I (2012) Interspecific interactions: a case study using the tuatara-fairy prion association. Ph.D. Thesis, Victoria University of Wellington, New Zealand

Cree A (1994) Low annual reproductive output in female reptiles from New Zealand. New Zealand Journal of Zoology 21: 351-372

Cree A (2014) Tuatara: Biology and conservation of a venerable survivor. Canterbury University Press, University of Canterbury, Christchurch NZ.

Cree A, Cockrem JF, Guillette UJ (1992) Reproductive cycles of male and female tuatara (Sphenodon punctatus) on Stephens Island, New Zealand. Journal of Zoology (London) 226: 199217

Cree A, Daugherty CH, Hay JM (1995) Reproduction of a rare New Zealand reptile, the tuatara (Sphenodon punctatus), on rat-free and rat-inhabited islands. Conservation Biology 9: 373-383

Cree A, Daugherty CH, Schafer SF, Brown D (1991a) Nesting and clutch size of tuatara (Sphenodon guntheri) on North Brother Island, Cook Strait. Tuatara 31: 9-16

Cree A, Guillette UJ, Cockrem JF, Brown MA, Chambers GK (1990a) Absence of daily cycles in plasma sex steroid concentrations in male and female tuatara (Sphenodon punctatus) and the effects of acute capture stress on females. General and Comparative Endocrinology 79: 103-113

Cree A, Guillette LJ, Cockrem JF, Joss JMP (1990b) Effects of capture and temperature stresses on plasma steroid concentrations in male tuatara (Sphenodon punctatus). Journal of Experimental Zoology 253: 38-46

Cree A, Guillette LJ, Cockrem JJ (1991b) Identification of female tuatara in ovulatory condition using plasma sex steroid concentrations. New Zealand Journal of Zoology 18: 421-426

Cree A, Tyrrell CL (2001). Patterns of corticosterone secretion in tuatara (Sphenodon): Comparisons with other reptiles, and applications in conservation management. In Goos HJT, Rastogi RK, Vaudry H, Pierantoni R eds, Perspective in Comparative Endocrinology: Unity and Diversity (14th International Congress of Comparative Endocrinology), Sorrento, Italy, pp 433441 
Cree A, Tyrrell CL, Preest MR, Thorburn D, Guillette LJ (2003) Protecting embryos from stress: corticosterone effects and the corticosterone response to capture and confinement during pregnancy in a live-bearing lizard (Hoplodactylus maculatus). General and Comparative Endocrinology 134: 316-329

Creel S, Dantzer B, Goymann W, Rubenstein DR (2013) The ecology of stress: effects of the social environment. Functional Ecology 27: 66-80

Crespi EJ, Williams TD, Jessop TS, Delehanty B (2013) Life history and the ecology of stress: how do glucocorticoid hormones influence life-history variation in animals? Functional Ecology 27: 93-106

Crews D, Moore MC (1986) Evolution of mechanisms controlling mating behavior. Science 231: $121-125$

Cromarty P, Alderson S (2013) Translocation statistics (2002-2010), and the revised Department of Conservation translocation process. Notornis 60: 55-62

Crossin GT, Trathan PN, Phillips RA, Gorman KB, Dawson A, Sakamoto KQ, Williams TD (2012) Corticosterone predicts foraging behavior and parental care in Macaroni penguins. American Naturalist 180: E31-E41

Dahl E, Orizaola G, Winberg S, Laurila A (2012) Geographic variation in corticosterone response to chronic predator stress in tadpoles. Journal of Evolutionary Biology 25: 1066-1076

Dantzer B, Fletcher QE, Boonstra R, Sheriff MJ (2014) Measures of physiological stress: a transparent or opaque window into the status, management and conservation of species? Conservation Physiology 2:

Davis JR, DeNardo DF (2009) Water supplementation affects the behavioral and physiological ecology of Gila monsters (Heloderma suspectum) in the Sonoran desert. Physiological and Biochemical Zoology 82: 739-748

Davis JR, DeNardo DF (2010) Seasonal patterns of body condition, hydration state, and activity of Gila monsters (Heloderma suspectum) at a Sonoran desert site. Journal of Herpetology 44: 8393

Dawbin WH (1982) The tuatara, Sphenodon punctatus: aspects of life history, growth and longevity. In Newman DG ed, New Zealand Herpetology Vol. Occasional Publication No. 2, Wellington: New Zealand Wildlife Service, pp 237-250

Dayger CA, Cease AJ, Lutterschmidt DI (2013) Responses to capture stress and exogenous corticosterone vary with body condition in female red-sided garter snakes (Thamnophis sirtalis parietalis). Hormones and Behavior 64: 748-754

DeNardo D, Licht P (1993) Effects of corticosterone on social behaviour of male lizards. Hormones and Behavior 27: 184-199

DeNardo D, Sinervo B (1994) Effects of steroid hormone interaction on activity and home range size of male lizards. Hormones and Behavior 28: 273-287 
Dickens MJ, Delehanty DJ, Romero LM (2009) Stress and translocation: alterations in the stress physiology of translocated birds. Proceedings of the Royal Society B: Biological Sciences 276: 2051-2056

Dickens MJ, Delehanty DJ, Romero LM (2010) Stress: An inevitable component of animal translocation. Biological Conservation 143: 1329-1341

Dickens MJ, Romero LM (2013) A consensus endocrine profile for chronically stressed wild animals does not exist. General and Comparative Endocrinology 191: 177-189

Dixon PAF, Okereke SE, Enwelum MCN (1985) Influence of species, sex and drug pretreatment on the metabolism of metyrapone. Comparative Biochemistry and Physiology C Pharmacology Toxicology \& Endocrinology 81: 241-243

Drake KK, Nussear KE, Esque TC, Barber AM, Vittum KM, Medica PA, Tracy CR, Hunter KW (2012) Does translocation influence physiological stress in the desert tortoise? Animal Conservation 15: 560-570

Dufty AM, Clobert J, Moller AP (2002) Hormones, developmental plasticity and adaptation. Trends in Ecology \& Evolution 17: 190-196

Dunlap KD (1995) Hormonal and behavioral-responses to food and water-deprivation in a lizard (Sceloporus occidentalis) - Implications for assessing stress in a natural population Journal of Herpetology 29: 345-351

Dunlap KD (2006) Ontogeny and scaling of hematocrit and blood viscosity in Western fence lizards, Sceloporus occidentalis. Copeia: 535-538

Dunlap KD, Wingfield JC (1995) External and internal influences on indexes of physiological stress. 1. Seasonal and population variation in adrenocortical secretion of free-living lizards, Sceloporus occidentalis. Journal of Experimental Zoology 271: 36-46

Dupoue A, Angelier F, Lourdais O, Bonnet X, Brischoux F (2014) Effect of water deprivation on baseline and stress-induced corticosterone levels in the Children's python (Antaresia childreni). Comparative Biochemistry and Physiology a-Molecular \& Integrative Physiology 168: 11-16

Dupoue A, Brischoux F, Lourdais O, Angelier F (2013) Influence of temperature on the corticosterone stress-response: an experiment in the Children's python (Antaresia childreni). General and Comparative Endocrinology 193: 178-184

Ebensperger LA, Tapia D, Ramírez-Estrada J, León C, Soto-Gamboa M, Hayes LD (2013) Fecal cortisol levels predict breeding but not survival of females in the short-lived rodent, Octodon degus. General and Comparative Endocrinology 186: 164-171

Eikenaar C, Husak J, Escallón C, Moore IT (2012) Variation in testosterone and corticosterone in amphibians and reptiles: relationships with latitude, elevation, and breeding season length. American Naturalist 180: 642-654

Ewen J, Armstrong D, Parker K, Seddon P (2012) Reintroduction biology: Integrating science and management. Blackwell Publishing Ltd.

Fazio E, Ferlazzo A (2003) Evaluation of stress during transport. Veterinary Research Communications 27: 519-524 
Fazio E, Medica P, Bruschetta G, Ferlazzo A (2014) Do handling and transport stress influence adrenocortical response in tortoises (Testudo hermanni)? ISRN Veterinary Science 2014: 798273798273

Field A, Miles J, Field Z (2012) Discovering Statistics Using R. SAGE Publications Ltd, London UK.

Finkler MS (2006) Effects of temperature, sex, and gravidity on the metabolism of SmallMouthed salamanders, Ambystoma texanum, during the reproductive season. Journal of Herpetology 40: 103-106

Firth BT, Thompson MB, Kennaway DJ, Belan I (1989) Thermal sensitivity of reptilian melatonin rhythms: "cold" tuatara vs "warm" skink. American Journal of Physiology 256: R1160-R1162

Franceschini MD, Rubenstein DI, Low B, Romero LM (2008) Fecal glucocorticoid metabolite analysis as an indicator of stress during translocation and acclimation in an endangered large mammal, the Grevy's zebra. Animal Conservation 11: 263-269

Freitak D, Bos N, Stucki D, Sundstrom L (2014) Inbreeding-related trade-offs in stress resistance in the ant Formica exsecta. Biology Letters 10:

French SS, DeNardo DF, Greives TJ, Strand CR, Demas GE (2010) Human disturbance alters endocrine and immune responses in the Galapagos marine iguana (Amblyrhynchus cristatus). Hormones and Behavior 58: 792-799

French SS, Fokidis HB, Moore MC (2008) Variation in stress and innate immunity in the tree lizard (Urosaurus ornatus) across an urban-rural gradient. Journal of Comparative Physiology BBiochemical Systemic and Environmental Physiology 178: 997-1005

Gaby MJ, Besson AA, Bezzina CN, Caldwell AJ, Cosgrove S, Cree A, Haresnape S, Hare KM (2011) Thermal dependence of locomotor performance in two cool-temperate lizards. Journal of Comparative Physiology. A, Sensory, Neural, and Behavioral Physiology 197: 869-875

Gans C, Gillingham JC, Clark DL (1984) Courtship, mating and male combat in tuatara, Sphenodon punctatus. Journal of Herpetology 18: 194-197

Garamszegi LZ, Eens M, Hurtrez-Bousses S, Moller AP (2005) Testosterone, testes size, and mating success in birds: a comparative study. Hormones and Behavior 47: 389-409

Gaston AJ, Scofield P (1995) Birds and tuatara on North Brother Island, Cook Strait, New Zealand. Notornis 42: 27-41

Gaze P (2001). Tuatara recovery plan 2001-2011, Threatened Species Recovery Plan 47, Department of Conservation, Nelson, pp 37

Gelling M, Johnson PJ, Moorhouse TP, Macdonald DW (2012) Measuring animal welfare within a reintroduction: an assessment of different indices of stress in water voles Arvicola amphibius. PLOS ONE 7:

Gerber G, Keener L, Jezier B, Czekala N, MacDonald E, Alberts A (2004). Effects of translocation on the blood chemistry, hematology, and endocrinology of critically endangered Turks and Caicos iguanas, (Cyclura carinata), American Association of Zoo Veterinarians, pp 568 
Germano JM, Bishop PJ (2009) Suitability of amphibians and reptiles for translocation. Conservation Biology 23: 7-15

Gillingham JC, Carmichael C, Miller T (1995) Social Behaviour of the tuatara, Sphenodon punctatus. Herpetological Monographs 9: 5-16

Gillooly JF, Brown JH, West GB, Savage VM, Charnov EL (2001) Effects of size and temperature on metabolic rate. Science 293: 2248-2251

Girling JE, Jones SM (2006) In vitro steroid production by adrenals and kidney-gonads from embryonic southern snow skinks (Niveoscincus microlepidotus): Implications for the control of the timing of parturition? General and Comparative Endocrinology 145: 169-176

Graham SP, Freidenfelds NA, McCormick GL, Langkilde T (2012) The impacts of invaders: basal and acute stress glucocorticoid profiles and immune function in native lizards threatened by invasive ants. General and Comparative Endocrinology 176: 400-408

Grayson KL, Mitchell NJ, Monks JM, Keall SN, Wilson JN, Nelson NJ (2014) Sex ratio bias and extinction risk in an isolated population of tuatara (Sphenodon punctatus). PLOS ONE 9:

Hamann M, Jessop TS, Limpus CJ, Whittier JM (2002) Interactions among endocrinology, seasonal reproductive cycles and the nesting biology of the female green sea turtle. Marine Biology 140: 823-830

Hamann M, Jessop TS, Schauble CS (2007) Fuel use and corticosterone dynamics in hatchling green sea turtles (Chelonia mydas) during natal dispersal. Journal of Experimental Marine Biology and Ecology 353: 13-21

Hare JR, Whitworth E, Cree A (2007) Correct orientation of a hand-held infrared thermometer is important for accurate measurement of body temperatures in small lizards and tuatara.

Herpetological Review 38: 311-315

Harrington LA, Moehrenschlager A, Gelling M, Atkinson RPD, Hughes J, Macdonald DW (2013) Conflicting and complementary ethics of animal welfare considerations in reintroductions. Conservation Biology 27: 486-500

Hartup BK, Olsen GH, Czekala NM (2005) Fecal corticoid monitoring in whooping cranes (Grus americana) undergoing reintroduction. Zoo Biology 24: 15-28

Hau M, Ricklefs RE, Wikelski M, Lee KA, Brawn JD (2010) Corticosterone, testosterone and lifehistory strategies of birds. Proceedings of the Royal Society B-Biological Sciences 277: 3203-3212

Hay JM, Sarre SD, Lambert DM, Allendorf FW, Daugherty CH (2010) Genetic diversity and taxonomy: a reassessment of species designation in tuatara (Sphenodon: Reptilia). Conservation Genetics 11: 1063-1081

Hayward LS, Wingfield JC (2004) Maternal corticosterone is transferred to avian yolk and may alter offspring growth and adult phenotype. General and Comparative Endocrinology 135: 365371

Heiken K (2013) The behavioral and physiological effects of long-distance translocation on Western rattlesnakes (Crotalus oreganus). MSc, California Polytechnic State University, San Luis Obispo 
Hews DK, Baniki AJA (2013) The breeding season duration hypothesis: acute handling stress and total plasma concentrations of corticosterone and androgens in male and female striped plateau lizards (Sceloporus virgatus). Journal of Comparative Physiology B Biochemical Systemic and Environmental Physiology 183: 933-946

\section{Hitchmough R, Anderson P, Barr B, Monks J, Lettink M, Reardon J, Tocher M, Whitaker T} (2013). Conservation status of New Zealand reptiles, 2012, New Zealand Threat Classification Series Vol. 2, pp 16

Holding ML, Frazier JA, Dorr SW, Henningsen SN, Moore IT, Taylor EN (2014a) Physiological and behavioral effects of repeated handling and short-distance translocations on free-ranging Northern Pacific rattlesnakes (Crotalus oreganus oreganus). Journal of Herpetology 48: 233-239

Holding ML, Frazier JA, Dorr SW, Pollock NB, Muelleman PJ, Branske A, Henningsen SN, Eikenaar C, Escallon C, Montgomery CE et al. (2014b) Wet- and dry-season steroid hormone profiles and stress reactivity of an insular dwarf snake, the Hog Island boa (Boa constrictor imperator). Physiological and Biochemical Zoology 87: 363-373

Jachowski DS, Slotow R, Millspaugh JJ (2013) Delayed physiological acclimatization by African elephants following reintroduction. Animal Conservation 16: 575-583

Jasnic N, Djordjevic J, Vujovic P, Lakic I, Djurasevic S, Cvijic G (2013) The effect of vasopressin $1 \mathrm{~b}$ receptor (V1bR) blockade on HPA axis activity in rats exposed to acute heat stress. Journal of Experimental Biology 216: 2302-2307

Jessop T, Sumner J, Lance V, Limpus C (2004a) Reproduction in shark-attacked sea turtles is supported by stress-reduction mechanisms. Proceedings of the Royal Society Biological Sciences Series B 271: S91-S94

Jessop TS (2001) Modulation of the adrenocortical stress response in marine turtles (Cheloniidae): evidence for a hormonal tactic maximizing maternal reproductive investment. Journal of Zoology 254: 57-65

Jessop TS, FitzSimmons NN, Limpus CJ, Whittier JM (1999a) Interactions between behavior and plasma steroids within the scramble mating system of the promiscuous green turtle, Chelonia mydas. Hormones and Behavior 36: 86-97

Jessop TS, Hamann M (2004) Hormonal and metabolic responses to nesting activities in the green turtle, Chelonia mydas. Journal of Experimental Marine Biology and Ecology 308: 253-267

Jessop TS, Hamann M, Limpus CJ (2004b) Body condition and physiological changes in male green turtles during breeding. Marine Ecology Progress Series 276: 281-288

Jessop TS, Hamann M, Read MA, Limpus CJ (2000) Evidence for a hormonal tactic maximizing green turtle reproduction in response to a pervasive ecological stressor. General and Comparative Endocrinology 118: 407-417

Jessop TS, Limpus CJ, Whittier JM (1999b) Plasma steroid interactions during high-density green turtle nesting and associated disturbance. General and Comparative Endocrinology 115: 90-100

Jessop TS, Sumner JM, Limpus CJ, Whittier JM (2004c) Interplay between plasma hormone profiles, sex and body condition in immature hawksbill turtles (Eretmochelys imbricata) 
subjected to a capture stress protocol. Comparative Biochemistry and Physiology, Part A: Molecular \& Integrative Physiology 137: 197-204

Jessop TS, Woodford R, Symonds MRE (2013) Macrostress: do large-scale ecological patterns exist in the glucocorticoid stress response of vertebrates? Functional Ecology 27: 120-130

Ji SN, Yang LL, Ge XF, Wang BJ, Cao J, Hu DF (2013) Behavioural and physiological stress responses to transportation in a group of Przewalski's horses (Equus ferus prezwalskii) Journal of Animal and Plant Sciences 23: 1077-1084

Jones HP, Tershy BR, Zavaleta ES, Croll DA, Keitt BS, Finkelstein ME, Howald GR (2008) Severity of the effects of invasive rats on seabirds: A global review. Conservation Biology 22: 16-26

Jones MEH (2008) Skull shape and feeding strategy in Sphenodon and other Rhynchocephalia (Diapsida : Lepidosauria). Journal of Morphology 269: 945-966

Jones MEH, Cree A (2012) Tuatara. Current Biology 22: R986-R987

Jones RE, Guillette $\amalg$ (1982) Hormonal control of oviposition and parturition in lizards. Herpetologica 38: 80-93

Jones SM, Bell K (2004) Plasma corticosterone concentrations in males of the skink Egernia whitii during acute and chronic confinement, and over a diel period. Comparative Biochemistry and Physiology a-Molecular \& Integrative Physiology 137: 105-113

Kindermann C, Narayan EJ, Hero J-M (2012) Urinary corticosterone metabolites and chytridiomycosis disease prevalence in a free-living population of male Stony Creek frogs (Litoria wilcoxii). Comparative Biochemistry and Physiology a-Molecular \& Integrative Physiology 162: 171-176

Kitaysky AS, Piatt JF, Wingfield JC (2007) Stress hormones link food availability and population processes in seabirds. Marine Ecology Progress Series 352: 245-258

Klose SM, Smith CL, Denzel AJ, Kalko EKV (2006) Reproduction elevates the corticosterone stress response in common fruit bats. Journal of Comparative Physiology a-Neuroethology Sensory Neural and Behavioral Physiology 192: 341-350

Klukowski M (2011) Effects of breeding season, testosterone and ACTH on the corticosterone response of free-ranging male fence lizards (Sceloporus undulatus). General and Comparative Endocrinology 173: 295-302

Knapp R, Moore MC (1995) Hormonal responses to aggression vary in different types of agonistic encounters in male Tree lizards, Urosaurus ornatus. Hormones and Behavior 29: 85-105

Kuznetsova A (2013) ImerTest: Tests for random and fixed effects for linear mixed effect models (Imer objects of Ime4 package)

Lancaster LT, Hazard LC, Clobert J, Sinervo BR (2008) Corticosterone manipulation reveals differences in hierarchical organization of multidimensional reproductive trade-offs in $r$ strategist and K-strategist females. Journal of Evolutionary Biology 21: 556-565 
Lance VA, Elsey RM, Butterstein G, Trosclair PL, III, Merchant M (2010) The effects of Hurricane Rita and subsequent drought on alligators in southwest Louisiana. Journal of Experimental Zoology Part A-Ecological Genetics and Physiology 313A: 106-113

Landys MM, Ramenofsky M, Wingfield JC (2006) Actions of glucocorticoids at a seasonal baseline as compared to stress-related levels in the regulation of periodic life processes. General and Comparative Endocrinology 148: 132-149

Langkilde T, Shine R (2006) How much stress do researchers inflict on their study animals? A case study using a scincid lizard, Eulamprus heatwolei. The journal of experimental biology 209: 1035-1043

Langkilde T, Trompeter WP (2011) Invader danger: lizards faced with novel predators exhibit altered behavioral stress responses. Integrative and Comparative Biology 51: E76-E76

Larson S, Monson D, Ballachey B, Jameson R, Wasser SK (2009) Stress-related hormones and genetic diversity in sea otters (Enhydra lutris). Marine Mammal Science 25: 351-372

Lawrence $\mathbf{M}$ (1997) The effect of temperature on the eating behaviour, gut passage times, and digestion efficiency of lizards. New Zealand Journal of Zoology 24: 328 (abstract)

Lendvai AZ, Giraudeau M, Chastel $\mathbf{O}$ (2007) Reproduction and modulation of the stress response: an experimental test in the house sparrow. Proceedings of the Royal Society Biological Sciences Series B 274: 391-397

Li C, Gu Y, Tang S, Fang H, Jiang G, Jiang Z (2011) Effects of acute low temperature stress on the endocrine reactions of the Qinghai toad-headed lizard. Current Zoology 57: 775-780

Li D, Wang G, Wingfield JC, Lei F (2012) A comparison of the adrenocortical responses to acute stress in cardueline finches from the Tibetan Plateau, Arctic Alaska and lowland Western North America. Journal of Ornithology 153: 761-770

Lillywhite HB, Heatwole H, Sheehy CM, III (2014) Dehydration and drinking behavior of the marine file snake Acrochordus granulatus. Physiological and Biochemical Zoology 87: 46-55

Lourdais O, Heulin B, Denardo DF (2008) Thermoregulation during gravidity in the Children's python (Antaresia childreni): a test of the preadaptation hypothesis for maternal thermophily in snakes. Biological Journal of the Linnean Society 93: 499-508

Love OP, McGowan PO, Sheriff MJ (2013) Maternal adversity and ecological stressors in natural populations: the role of stress axis programming in individuals, with implications for populations and communities. Functional Ecology 27: 81-92

Love OP, Wynne-Edwards KE, Bond L, Williams TD (2008) Determinants of within- and amongclutch variation in yolk corticosterone in the European starling. Hormones and Behavior 53: 104111

Lucas LD, French SS (2012) Stress-induced tradeoffs in a free-living lizard across a variable landscape: consequences for individuals and populations. PLOS ONE 7:

MacDougall-Shackleton SA, Schmidt KL, Furlonger AA, MacDougall-Shackleton EA (2013) HPA axis regulation, survival, and reproduction in free-living sparrows: Functional relationships or developmental correlations? General and Comparative Endocrinology 190: 188-193 
Maclean GS, Lee AK, Withers PC (1975) Hematological adjustments with diurnal changes in body temperatures in a lizard and a mouse. Comparative Biochemistry and Physiology. Part A: Molecular and Integrative Physiology 51: 241-249

Madliger CL, Love OP (2014) The need for a predictive, context-dependent approach to the application of stress hormones in conservation. Conservation Biology 28: 283-287

Mahmoud IY, Vliet K, Guillette L, Plude JL (1996) Effect of stress and ACTH(1-24) on hormonal levels in male alligators, Alligator mississippiensis. Comparative Biochemistry and Physiology $A$ 115: $57-62$

Manire CA, Rasmussen LEL, Gelsleichter J, Hess DL (2004) Maternal serum and yolk hormone concentrations in the placental viviparous bonnethead shark, Sphyrna tiburo. General and Comparative Endocrinology 136: 241-247

Markwell TJ, Daugherty CH (2002) Invertebrate and lizard abundance is greater on seabirdinhabited islands than on seabird-free islands in the Marlborough Sounds, New Zealand. Ecoscience 9: 293-299

Martin LB, Brace AJ, Urban A, Coon CAC, Liebl AL (2012) Does immune suppression during stress occur to promote physical performance? Journal of Experimental Biology 215: 4097-4103

Meylan S, Clobert J (2004) Maternal effects on offspring locomotion: Influence of density and corticosterone elevation in the lizard Lacerta vivipara. Physiological and Biochemical Zoology 77: 450-458

Meylan S, Haussy C, Voituron Y (2010) Physiological actions of corticosterone and its modulation by an immune challenge in reptiles. General and Comparative Endocrinology 169: 158-166

Michel CL, Chaste O, Bonnet X (2011) Ambient temperature and pregnancy influence cortisol levels in female guinea pigs and entail long-term effects on the stress response of their offspring. General and Comparative Endocrinology 171: 275-282

Michel CL, Pastore J-H, Bonnet X (2013) Impact of cool versus warm temperatures on gestation in the Aspic viper (Vipera aspis). Comparative Biochemistry and Physiology A 165: 338-342

Miller KA, Miller HC, Moore JA, Mitchell NJ, Cree A, Allendorf FW, Sarre SD, Keall SN, Nelson NJ (2012) Securing the demographic and genetic future of tuatara through assisted colonization. Conservation Biology 26: 790-798

Mitchell NJ, Allendorf FW, Keall SN, Daugherty CH, Nelson NJ (2010) Demographic effects of temperature-dependent sex determination: will tuatara survive global warming? Global Change Biology 16: 60-72

Mitchell NJ, Nelson NJ, Cree A, Pledger S, Keall SN, Daugherty CH (2006) Support for a rare pattern of temperature-dependent sex determination in archaic reptiles: evidence from two species of tuatara (Sphenodon). Frontiers in Zoology 3: 9

Moore IT, Greene MJ, Lerner DT, Asher CE, Krohmer RW, Hess DL, Whittier J, Mason RT (2005) Physiological evidence for reproductive suppression in the introduced population of brown tree snakes (Boiga irregularis) on Guam. Biological Conservation 121: 91-98 
Moore IT, Greene MJ, Mason RT (2001) Environmental and seasonal adaptations of the adrenocortical and gonadal responses to capture stress in two populations of the male garter snake, Thamnophis sirtalis. Journal of Experimental Zoology 289: 99-108

Moore IT, Jessop TS (2003) Stress, reproduction, and adrenocortical modulation in amphibians and reptiles. Hormones and Behavior 43: 39-47

Moore IT, Lemaster MP, Mason RT (2000a) Behavioural and hormonal responses to capture stress in the male red-sided garter snake, Thamnophis sirtalis parietalis. Animal Behaviour 59: 529-534

Moore IT, Lerner JP, Lerner DT, Mason RT (2000b) Relationships between annual cycles of testosterone, corticosterone, and body condition in male red-spotted garter snakes, Thamnophis sirtalis concinnus. Physiological and Biochemical Zoology 73: 307-312

Moore JA (2008) Fitness implications of the mating system and reproductive ecology of tuatara. $\mathrm{PhD}$, Victoria University of Wellington

Moore JA, Daugherty CH, Godfrey SS, Nelson NJ (2009a) Seasonal monogamy and multiple paternity in a wild population of a territorial reptile (tuatara). Biological Journal of the Linnean Society 98: 161-170

Moore JA, Daugherty CH, Nelson NJ (2009b) Large male advantage: phenotypic and genetic correlates of territoriality in tuatara. Journal of Herpetology 43: 570-578

Moore JA, Grant T, Brown D, Keall SN, Nelson NJ (2010) Mark-recapture accurately estimates census for tuatara, a burrowing reptile. Journal of Wildlife Management 74: 897-901

Narayan E, Hero J-M (2011) Urinary corticosterone responses and haematological stress indicators in the endangered Fijian ground frog (Platymantis vitiana) during transportation and captivity. Australian Journal of Zoology 59: 79-85

Narayan EJ, Cockrem JF, Hero J-M (2011a) Urinary corticosterone metabolite responses to capture and captivity in the cane toad (Rhinella marina). General and Comparative Endocrinology 173: 371-377

Narayan EJ, Cockrem JF, Hero J-M (2012) Effects of temperature on urinary corticosterone metabolite responses to short-term capture and handling stress in the cane toad (Rhinella marina). General and Comparative Endocrinology 178: 301-305

Narayan EJ, Hero J-M (2014) Acute thermal stressor increases glucocorticoid response but minimizes testosterone and locomotor performance in the Cane toad (Rhinella marina). PLoS ONE 9: e92090-e92090

Narayan EJ, Molinia FC, Cockrem JF, Hero J-M (2011b) Changes in urinary testosterone and corticosterone metabolites during short-term confinement with repeated handling in wild male cane toads (Rhinella marina). Australian Journal of Zoology 59: 264-269

Nelson NJ, Keall SN, Pledger S, Daugherty CH (2002) Male-biased sex ratio in a small tuatara population. Journal of Biogeography 29: 633-640 
Newman DG (1987) Burrow use and population densities of tuatara (Sphenodon punctatus ) and how they are influenced by fairy prions (Pachyptila turtur) on Stephens Island, New Zealand. Herpetologica 43: 336-344

Newman DG, Watson PR, McFadden I (1994) Egg production by tuatara on Lady Alice and Stephens Island, New Zealand. New Zealand Journal of Zoology 21: 387-398

NIWA (2014) National Institute of Water and Atmospheric Research www.niwa.co.nz

Owen DAS, Carter ET, Holding ML, Islam K, Moore IT (2014) Roads are associated with a blunted stress response in a North American pit viper. General and Comparative Endocrinology 202: 87-92

Parker KA, Dickens MJ, Clarke RH, Lovegrove TG (2012) The theory and practice of catching, holding, moving and releasing animals, Reintroduction Biology: John Wiley \& Sons, Ltd, pp 105137

Phillips JB, Klukowski M (2008) Influence of season and adrenocorticotropic hormone on corticosterone in free-living female Eastern fence lizards (Sceloporus undulatus). Copeia: 570578

Polo-Cavia N, Lopez P, Martin J (2012) Effects of body temperature on righting performance of native and invasive freshwater turtles: Consequences for competition. Physiology \& Behavior 108: $28-33$

Porteous A, Mullan B (2013). The 2012 - 13 drought: an assessment and historical perspective, Ministry for Primary Industries, Wellington, New Zealand, pp 57

Preest MR, Cree A (2008) Corticosterone treatment has subtle effects on thermoregulatory behavior and raises metabolic rate in the New Zealand Common gecko, Hoplodactylus maculatus. Physiological and Biochemical Zoology 81: 641-650

Preest MR, Cree A, Tyrrell CL (2005) ACTH-induced stress response during pregnancy in a viviparous gecko: no observed effect on offspring quality. Journal of Experimental Zoology Part $A$ Comparative Experimental Biology 303A: 823-835

Quirici V, Venegas CI, Gonzalez-Gomez PL, Castano-Villa GJ, Wingfield JC, Vasquez RA (2014) Baseline corticosterone and stress response in the Thorn-tailed Rayadito (Aphrastura spinicauda) along a latitudinal gradient. General and Comparative Endocrinology 198: 39-46

Radder RS, Elphick MJ, Warner DA, Pike DA, Shine R (2008) Reproductive modes in lizards: measuring fitness consequences of the duration of uterine retention of eggs. Functional Ecology 22: 332-339

Ray PP, Chaudhuri-Sengupta S, Maiti BR (2004) Adrenocortical involvement during diverse stress in soft-shelled turtle Lissemys p. punctata Bonnoterre. Indian Journal of Experimental Biology 42: 620-625

Ray PP, Sengupta A, Chaudhuri-Sengupta S, Maiti BR (2008) Thyroidal inhibition following diverse stress in soft-shelled turtles, Lissemys punctata punctata Acta Biologica Hungarica 59: 403-412 
Reed DH, Lowe EH, Briscoe DA, Frankham R (2003) Fitness and adaptation in a novel environment: Effect of inbreeding, prior environment, and lineage. Evolution 57: 1822-1828

Refsnider JM, Daugherty CH, Godfrey SS, Keall SN, Moore JA, Nelson NJ (2013) Patterns of nesting migrations in the tuatara (Sphenodon punctatus), a colonially nesting island reptile. Herpetologica 69: 282-290

Refsnider JM, Daugherty CH, Keall SN, Nelson NJ (2010) Nest-site choice and fidelity in tuatara on Stephens Island, New Zealand. Journal of Zoology 280: 396-402

Reimers TJ, McCann JP, Cowan RG (1983) Effects of storage times and temperatures on T3, T4, $\mathrm{LH}$, prolactin, insulin, cortisol and progesterone concentrations in blood-samples from cows. Journal of Animal Science 57: 683-691

Rhen T, Crews D, Fivizzani A, Elf P (2006) Reproductive tradeoffs and yolk steroids in female leopard geckos, Eublepharis macularius. Journal of Evolutionary Biology 19: 1819-1829

Riechert J, Chastel O, Becker PH (2012) Why do experienced birds reproduce better? Possible endocrine mechanisms in a long-lived seabird, the common tern. General and Comparative Endocrinology 178: 391-399

Robert KA, Vleck C, Bronikowski AM (2009) The effects of maternal corticosterone levels on offspring behavior in fast- and slow-growth garter snakes (Thamnophis elegans). Hormones and Behavior 55: 24-32

Rödl T, Berger S, Michael Romero L, Wikelski M (2007) Tameness and stress physiology in a predator-naive island species confronted with novel predation threat. Proceedings of the Royal Society B: Biological Sciences 274: 577-582

Romero LM (2002) Seasonal changes in plasma glucocorticoid concentrations in free-living vertebrates. General and Comparative Endocrinology 128: 1-24

Romero LM (2004) Physiological stress in ecology: lessons from biomedical research. Trends in Ecology \& Evolution 19: 249-255

Romero LM (2006) Seasonal changes in hypothalamic-pituitary-adrenal axis sensitivity in freeliving house sparrows (Passer domesticus). General and Comparative Endocrinology 149: 66-71

Romero LM, Wikelski M (2001) Corticosterone levels predict survival probabilities of Galápagos marine iguanas during El Niño events. Proceedings of the National Academy of Sciences of the United States of America 98: 7366-7370

Romero LM, Wikelski M (2002) Exposure to tourism reduces stress-induced corticosterone levels in Galápagos marine iguanas. Biological Conservation 108: 371-374

Romero LM, Wikelski M (2006) Diurnal and nocturnal differences in hypothalamic-pituitaryadrenal axis function in Galápagos marine iguanas. General and Comparative Endocrinology 145: 177-181

Romero LM, Wikelski M (2010) Stress physiology as a predictor of survival in Galapagos marine iguanas. Proceedings of the Royal Society B-Biological Sciences 277: 3157-3162 
Rubenstein DR, Wikelski M (2005) Steroid hormones and aggression in female Galápagos marine iguanas. Hormones and Behavior 48: 329-341

Saino N, Romano M, Ferrari RP, Martinelli R, Moller AP (2005) Stressed mothers lay eggs with high corticosterone levels which produce low-quality offspring. Journal of Experimental Zoology Part a-Comparative Experimental Biology 303A: 998-1006

Saint-Girons H, Bell BD, Newman DG (1981) Brief observations on the activity and thermoregulation of the tuatara, Sphenodon punctatus . (Reptilia: Rhynchocephalia), on Stephens Island, Cook Strait, New Zealand. Journal of Zoology, London:

Saint Girons H (1980) Thermoregulation in reptiles with special reference to the tuatara and its ecophysiology. Tuatara 24: 59-80

Saint Girons H, Bell BD, Newman DG (1980) Observations on the activity and thermoregulation of the tuatara, Sphenodon punctatus (Reptilia: Rhynchocephalia), on Stephens Island. New Zealand Journal of Zoology 7: 551-556

Saint Girons H, Newman DG (1987) The reproductive cycle of the male tuatara, Sphenodon punctatus, on Stephens Island, New Zealand. New Zealand Journal of Zoology 14: 231-237

Sapolsky RM, Romero LM, Munck AU (2000) How do glucocorticoids influence stress responses? Integrating permissive, suppressive, stimulatory, and preparative actions. Endocrine Reviews 21: 55-89

Sarrieau A, Chaouloff F, Lemaire V, Mormede P (1998) Comparison of the neuroendocrine responses to stress in outbred, inbred and F1 hybrid rats. Life Sciences 63: 87-96

Schoech SJ, Romero LM, Moore IT, Bonier F (2013) Constraints, concerns and considerations about the necessity of estimating free glucocorticoid concentrations for field endocrine studies. Functional Ecology 27: 1100-1106

Scholnick DA, Weinstein RB, Gleeson TT (1997) The influence of corticosterone and glucagon on metabolic recovery from exhaustive exercise in the desert iguana Dipsosaurus dorsalis. General and Comparative Endocrinology 106: 147-154

Schramm BG, Casares M, Lance VA (1999) Steroid levels and reproductive cycle of the Galapagos tortoise, Geochelone nigra, living under seminatural conditions on Santa Cruz Island (Galapagos). General and Comparative Endocrinology 114: 108-120

Schulte-Hostedde Al, Zinner B, Millar JS, Hickling GJ (2005) Restitution of mass-size residuals: validating body condition indices. Ecology 86: 155-163

Schultner J, Kitaysky AS, Welcker J, Hatch S (2013) Fat or lean: adjustment of endogenous energy stores to predictable and unpredictable changes in allostatic load. Functional Ecology 27: 45-55

Seddon PJ, Griffiths CJ, Soorae PS, Armstrong DP (2014) Reversing defaunation: Restoring species in a changing world. Science 345: 406-412

Seebacher F, Grigg GC, Beard LA (1999) Crocodiles as dinosaurs: behavioural thermoregulation in very large ectotherms leads to high and stable body temperatures. Journal of Experimental Biology 202: 77-86 
Selman W, Jawor JM, Qualls CP (2012) Seasonal variation of corticosterone levels in Graptemys flavimaculata, an imperiled freshwater turtle. Copeia: 698-705

Sheriff MJ, Dantzer B, Delehanty B, Palme R, Boonstra R (2011) Measuring stress in wildlife: techniques for quantifying glucocorticoids. Oecologia 166: 869-887

Silverin B, Arvidsson B, Wingfield J (1997) The adrenocortical responses to stress in breeding Willow Warblers Phylloscopus trochilus in Sweden: effects of latitude and gender. Functional Ecology 11: 376-384

Sinervo B, Miles DB (2011) Chapter 8 - Hormones and Behavior of Reptiles. In Lopez DONH ed, Hormones and Reproduction of Vertebrates, London: Academic Press, pp 215-246

Smith CF, Schuett GW, Hoss SK (2012) Reproduction in female copperhead snakes (Agkistrodon contortrix): plasma steroid profiles during gestation and post-birth periods. Zoological Science 29: $273-279$

Socorro Aguilar-Cucurachi MA, Dias PAD, Rangel-Negrin A, Chavira R, Boeck L, CanalesEspinosa D (2010) Preliminary evidence of accumulation of stress during translocation in Mantled Howlers. American Journal of Primatology 72: 805-810

Spee M, Beaulieu M, Dervaux A, Chastel O, Le Maho Y, Raclot T (2010) Should I stay or should I go? Hormonal control of nest abandonment in a long-lived bird, the Adelie penguin. Hormones and Behavior 58: 762-768

Stebbins RC (1958) An experimental study of the "third eye" of the tuatara. Copeia: 183-190

Strasser EH, Heath JA (2013) Reproductive failure of a human-tolerant species, the American kestrel, is associated with stress and human disturbance. Journal of Applied Ecology 50: 912-919

Summers CH, Norman MF (1988) Chronic low humidity-stress in the lizard Anolis carolinensis Changes in diurnal corticosterone rhythyms Journal of Experimental Zoology 247: 271-278

Sykes KL, Klukowski M (2009) Effects of acute temperature change, confinement and housing on plasma corticosterone in water snakes, Nerodia sipedon (Colubridae: Natricinae). Journal of Experimental Zoology Part A-Ecological Genetics and Physiology 311A: 172-181

Taillon J, Brodeur V, Festa-Bianchet M, Cote SD (2011) Variation in body condition of migratory caribou at calving and weaning: Which measures should we use? Ecoscience 18: 295-303

Tamplin JW, Moran VF, Riesberg EJ (2013) Response of juvenile Diamond-Backed terrapins (Malaclemys terrapin) to an aquatic thermal gradient. Journal of Thermal Biology 38: 434-439

Tarszisz E, Dickman CR, Munn AJ (2014) Physiology in conservation translocations. Conservation Physiology 2:

Tartu S, Angelier F, Herzke D, Moe B, Bech C, Gabrielsen GW, Bustnes JO, Chastel O (2014) The stress of being contaminated? Adrenocortical function and reproduction in relation to persistent organic pollutants in female black legged kittiwakes. The Science of the total environment 476-

477: $553-560$ 
Taylor DP, Vradenburg JN, Smith LM, Lovern MB, McMurry ST (2014) Effects of anthropogenic and environmental stress on the corticosterone levels of wintering Northern Pintails (Anas acuta). Canadian Journal of Zoology 92: 185-193

Teixeira CP, de Azevedo CS, MendI M, Cipreste CF, Young RJ (2007) Revisiting translocation and reintroduction programmes: the importance of considering stress. Animal Behaviour 73: 1-13

Telemeco RS, Addis EA (2014) Temperature has species-specific effects on corticosterone in alligator lizards. General and Comparative Endocrinology 206: 184-192

Thaker M, Vanak AT, Lima SL, Hews DK (2010) Stress and aversive learning in a wild vertebrate: the role of corticosterone in mediating escape from a novel stressor. American Naturalist 175: $50-60$

Thierry A-M, Massemin S, Handrich Y, Raclot T (2013) Elevated corticosterone levels and severe weather conditions decrease parental investment of incubating Adelie penguins. Hormones and Behavior 63: 475-483

Thompson MB, Newman DG, Watson PR (1991) Use of oxytocin in obtaining eggs from tuatara (Sphenodon punctatus). Journal of Herpetology 25: 101-104

Tokarz RR, Summers CH (2011) Stress and Reproduction in Reptiles.

Towns DR (2009) Eradications as reverse invasions: lessons from Pacific rat (Rattus exulans) removals on New Zealand islands. Biological Invasions 11: 1719-1733

Towns DR, Broome KG (2003) From small Maria to massive Campbell: forty years of rat eradications from New Zealand islands. New Zealand Journal of Zoology 30: 377-398

Towns DR, Daugherty CH, Cromarty P (1990) Protocols for translocation of organisms to islands. In Towns DR, Daugherty CH, Atkinson IAE eds, Ecological Restoration of New Zealand Islands Vol. 2, Wellington: Department of Conservation, pp 240-254

Towns DR, Parrish GR, Tyrrell CL, Ussher GT, Cree A, Newman DG, Whitaker AT, Westbrooke I (2007) Responses of tuatara (Sphenodon punctatus) to removal of introduced Pacific rats from islands. Conservation Biology 21: 1021-1031

Tyrrell C (1993) Corticosterone cycles in wild and captive tuatara (Sphenodon punctatus) Unpublished M.Sc thesis, University of Otago, Dunedin

Tyrrell C, Cree A (1998) Relationships between corticosterone concentration and season, time of day and confinement in a wild reptile (tuatara, Sphenodon punctatus). General and Comparative Endocrinology 110: 97-108

Tyrrell C, Cree A, Towns DR (2000). Variation in reproduction of northern tuatara (Sphenodon punctatus punctatus) in the presence and absence of kiore. In $153 \mathrm{SfC}$ ed, pp 44

Uller T, Hollander J, Astheimer L, Olsson M (2009) Sex-specific developmental plasticity in response to yolk corticosterone in an oviparous lizard. Journal of Experimental Biology 212: 1087-1091 
Valverde RA, Owens DW, Mackenzie DS, Amoss MS (1999) Basal and stress-induced corticosterone levels in olive ridley sea turtles (Lepidochelys olivacea) in relation to their mass nesting behavior. Journal of Experimental Zoology 284: 652-662

van de Merwe JP, Ibrahim K, Whittier JM (2013) Post-emergence handling of green turtle hatchlings: improving hatchery management worldwide. Animal Conservation 16: 316-323

Walls GY (1981) Feeding ecology of the tuatara (Sphenodon punctatus) on Stephens Island, Cook Strait. New Zealand Journal of Ecology 4: 89-97

Warner DA, Radder RS, Shine R (2009) Corticosterone exposure during embryonic development affects offspring growth and sex ratios in opposing directions in two lizard species with environmental sex determination. Physiological and Biochemical Zoology 82: 363-371

Waye HL, Mason RT (2008) A combination of body condition measurements is more informative than conventional condition indices: Temporal variation in body condition and corticosterone in brown tree snakes (Boiga irregularis). General and Comparative Endocrinology 155: 607-612

Werner YL, Whitaker AH (1978) Observations and comments on the body temperatures of some New Zealand reptiles. New Zealand Journal of Zoology 5: 375-393

Whirledge S, Cidlowski JA (2013) A role for glucocorticoids in stress-impaired reproduction: Beyond the hypothalamus and pituitary. Endocrinology 154: 4450-4468

Wikelski M, Cooke SJ (2006) Conservation physiology. Trends in Ecology \& Evolution 21: 38-46

Wikelski M, Romero LM (2003) Body size, performance and fitness in Galápagos marine iguanas. Integrative and Comparative Biology 43: 376-386

Wilson DS, Nagy KA, Tracy CR, Morafka DJ, Yates RA (2001) Water balance in neonate and juvenile desert tortoises, Gopherus agassizii. Herpetological Monographs 15: 158-170

Wilson J (2010) Population viability and resource competition on North Brother Island: Conservation implications for tuatara (Sphenodon punctatus) and Duvaucel"s gecko (Hoplodactylus duvaucelii). MSc, Victoria University of Wellington

Wingfield JC (2013) Ecological processes and the ecology of stress: the impacts of abiotic environmental factors. Functional Ecology 27: 37-44

Wingfield JC, Deviche P, Sharbaugh S, Astheimer LB, Holberton R, Suydam R, Hunt K (1994) Seasonal changes of the adrenocortical responses to stress in Redpolls, Acanthis flammea, in Alaska. Journal of Experimental Zoology 270: 372-380

Wingfield JC, Hegner RE, Dufty AM, Ball GF (1990) The 'challenge hypothesis' - theoretical implications for patterns of testosterone secretion, mating systems, and breeding strategies. American Naturalist 136: 829-846

Wingfield JC, Kitaysky AS (2002) Endocrine responses to unpredictable environmental events: stress or anti-stress hormones? Integrative and Comparative Biology 42: 600-609 
Wingfield JC, Maney DL, Breuner CW, Jacobs JD, Lynn S, Ramenofsky M, Richardson RD (1998) Ecological bases of hormone-behavior interactions: The "emergency life history stage". American Zoologist 38: 191-206

Wingfield JC, Sapolsky RM (2003) Reproduction and resistance to stress: When and how. Journal of Neuroendocrinology 15: 711-724

Woodley SK, Moore MC (2002) Plasma corticosterone response to an acute stressor varies according to reproductive condition in female tree lizards (Urosaurus ornatus). General and Comparative Endocrinology 128: 143-148

Woodley SK, Painter DL, Moore MC, Wikelski M, Romero LM (2003) Effect of tidal cycle and food intake on the baseline plasma corticosterone rhythm in intertidally foraging marine iguanas. General and Comparative Endocrinology 132: 216-222

Yang EJ, Wilczynski W (2003) Interaction effects of corticosterone and experience on aggressive behavior in the green anole lizard. Hormones and Behavior 44: 281-292

Zhang S, Lei F, Liu S, Li D, Chen C, Wang P (2011) Variation in baseline corticosterone levels of Tree Sparrow (Passer montanus) populations along an urban gradient in Beijing, China. Journal of Ornithology 152: 801-806

Zidon R, Saltz D, Shore LS, Motro U (2009) Behavioral changes, stress, and survival following reintroduction of Persian Fallow deer from two breeding facilities. Conservation Biology 23: 1026-1035 